\title{
MICROMETEOROID FLUENCE VARIATION IN CRITICAL ORBITS DUE TO ASTEROID DISRUPTION
}

\author{
A Thesis \\ Presented to \\ the Faculty of California Polytechnic State University \\ San Luis Obispo
}

In Partial Fulfillment

of the Requirements for the Degree

Master of Science in Aerospace Engineering

by

Eliot Dan Aretskin-Hariton

May 2013 
(C) 2013

Eliot Dan Aretskin-Hariton

ALL RIGHTS RESERVED 
COMMITTEE MEMBERSHIP

TITLE:

AUTHOR:

DATE SUBMITTED:

COMmitTeE CHAIR: Kira Abercromby, Ph.D., Assistant Professor of Aerospace Engineering, Cal Poly SLO

COMmitTeE MEMBER: Eric Mehiel, Ph.D., Associate Professor of Aerospace Engineering, Cal Poly SLO

COMMITTEE MEMBER: Robert Weaver, Ph.D., Fellow at Los Alamos National Laboratory

COMMITTEE MEMBER: Gary Hughes, Ph.D, Assistant Professor of Statistics, Cal Poly SLO 


\title{
Abstract \\ MICROMETEOROID FLUENCE VARIATION IN CRITICAL ORBITS DUE TO ASTEROID DISRUPTION
}

\author{
Eliot Dan Aretskin-Hariton
}

Micrometeoroid fluence resulting from asteroid disruption using nuclear munitions was analyzed to determine if this mitigation technique posed a significant threat to satellites in critical orbit regimes. Understanding the Micrometeoroid and Orbital Debris (MMOD) environment is critical for spacecraft design and survivability. Nuclear disruption techniques for mitigating hazardous asteroids may lead to an increase in micrometeoroid fluence in orbit regimes typically used by civilian and military satellites. A novel framework to assess the transient micrometeoroid fluence risk from disrupted asteroids is presented. This framework is capable of analyzing a wide range of asteroid disruption scenarios. The results from several example scenarios are presented. Transient fluence risk is highly scenario dependent and will vary from the examples provided herein. 


\title{
Acknowledgements
}

I would like to thank the following individuals for their strong contributions to this project: Dr. Kira Abercromby for her enthusiastic support of space environment research and her tireless work as my thesis advisor. Dr. Robert Weaver for supplying the initial velocity distribution curves and pictures which he created especially for this study. Dr. Gary Hughes for many long conversations about statistical methods. Dr. Eric Mehiel for his encouragement and support. Dr. Bong Wie, Dr. David Dearborn, and Brian Kaplinger for their work on disruption velocity curves which gave me the initial idea for this research. My wife Asya and our family for supporting me in this effort.

\author{
Real goodness comes \\ from the desire to help, \\ and a wealth of knowledge \\ of how not to do evil.
}




\section{Contents}

List of Tables $\quad$ ix

List of Figures $\quad x$

1 Introduction 1

2 Previous Work 9

2.1 Fragmentation . . . . . . . . . . . . . . . . 10

2.2 Disruption Modeling . . . . . . . . . . . . . . . 10

2.3 Trade Studies . . . . . . . . . . . . . . . . . . . . . . . . 12

2.4 Shielding . . . . . . . . . . . . . . . . . 13

2.5 Flux Models . . . . . . . . . . . . . . . 15

2.6 Mitigation Planning . . . . . . . . . . . . . . 16

2.7 Summary . . . . . . . . . . . . . . . . . 18

3 The Framework $\quad 19$

3.1 Overview of Modules . . . . . . . . . . . . . . . 21

3.1.1 Input Parameters . . . . . . . . . . . . . . . . . . 21

3.1.2 Orbital Propagation .................. 33

3.1.3 Critical Orbit Crossing Counter . . . . . . . . . . . . 37

3.1.4 Statistical Analyzer . . . . . . . . . . . . . . . . . . . 41

3.1.5 MMOD Fluence Analyzer . . . . . . . . . . . . . . . 44 
3.1.6 Output Parameters . . . . . . . . . . . . . . 47

3.2 Assumptions . . . . . . . . . . . . . . . . . . . . . . . . . . . 49

4 Program V and V 53

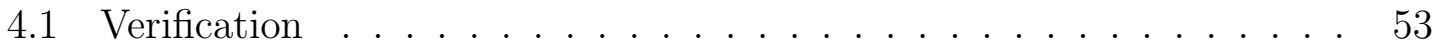

4.1.1 Propagator Module ............... 53

4.1.2 Critical Orbit Crossing . . . . . . . . . . . . 57

4.1.3 Velocity Distributions .............. 58

4.2 Validation . . . . . . . . . . . . . . . . 59

5 Example Disruptions $\quad 61$

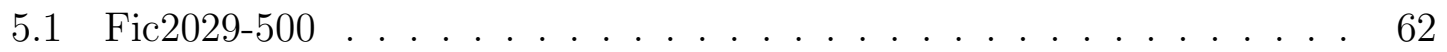

5.1 .1 Input Parameters . . . . . . . . . . . . . . . . 63

5.1.2 Results - April 9 Disruption . . . . . . . . . . . . 65

5.2 Fic2029-400 . . . . . . . . . . . . . . . . . . 74

5.2 .1 Input Parameters . . . . . . . . . . . . . . . 75

5.2.2 Results - April 9 Disruption . . . . . . . . . . . 75

5.3 Fic2029-600 . . . . . . . . . . . . . . . . . 77

5.3.1 Input Parameters . . . . . . . . . . . . . . . 78

5.3.2 Results - April 9 Disruption . . . . . . . . . . . 79

6 Conclusions $\quad 81$

7 Future Work $\quad 84$

8 Appendix $\quad 87$

8.1 Fic2029-500 Additional Critical Orbits . . . . . . . . . . . 87

8.1.1 Results - April 9 Disruption . . . . . . . . . . . 87

8.1.2 Results - April 11 Disruption . . . . . . . . . . . 95

8.2 Fic2029-400 . . . . . . . . . . . . . . . . 107

8.2.1 Results - April 9 Disruption . . . . . . . . . . . 107 


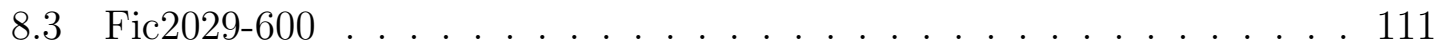

8.3.1 Results - April 9 Disruption . . . . . . . . . . . 111

8.4 Velocity Distributions . . . . . . . . . . . . . . . . . 115

8.5 Supplemental Tables . . . . . . . . . . . . . . . . 117

8.6 J2 J6 Coordinate System Transformation . . . . . . . . . . . . . 119

$\begin{array}{ll}\text { Bibliography } & 125\end{array}$

viii 


\section{List of Tables}

4.1 Results Comparison, Three Fic2029-500 Disruption Studies . . . . . . 60

5.1 Input Parameters, April 9th Disruption . . . . . . . . . . . . 64

5.2 Progress Report . . . . . . . . . . . . . . 65

5.3 Results Comparison, April 9th vs. April 11th, 2029, Disruption Studies 66

5.4 Input Parameters, April 9th Disruption . . . . . . . . . . . . . 75

5.5 Results Comparison, $500 \mathrm{kt}$ vs $400 \mathrm{kt}, 20 \mathrm{~m} \mathrm{LRC} \mathrm{.} \mathrm{.} \mathrm{.} \mathrm{.} \mathrm{.} \mathrm{.} \mathrm{.} \mathrm{.} \mathrm{.} \mathrm{.} 76$

5.6 Results Comparison, $500 \mathrm{kt} 20 \mathrm{~m} \mathrm{LRC} \mathrm{vs} 400 \mathrm{kt} 30 \mathrm{~m} \mathrm{LRC,} \mathrm{.} \mathrm{.} \mathrm{.} \mathrm{.} \mathrm{.} 77$

5.7 Event Duration Comparison, 500 kt vs 400 kt, 20 m LRC . . . . . . . 77

5.8 Input Parameters, April 9th Disruption . . . . . . . . . . . . . 78

5.9 CI Comparison, 500 kt vs 600 kt, $20 \mathrm{~m} \mathrm{LRC} \mathrm{.} \mathrm{.} \mathrm{.} \mathrm{.} \mathrm{.} \mathrm{.} \mathrm{.} \mathrm{.} \mathrm{.} \mathrm{.} \mathrm{.} \mathrm{.} 80$

5.10 Event Duration Comparison, 500 kt vs 600 kt, 20 m LRC . . . . . . . 80

5.11 Results Comparison, 500 kt 20 m LRC vs 400 kt 15 m LRC . . . . . 80

8.1 Input Parameters, April 11th Disruption . . . . . . . . . . . . . . 117

8.2 Input Parameters, April 9th Disruption . . . . . . . . . . . . . 118

8.3 Input Parameters, April 11th Disruption . . . . . . . . . . . . . . 119

8.4 Absolute Magnitude to Diameter Translation . . . . . . . . . . . . . . 123

8.5 Absolute Magnitude to Diameter Translation . . . . . . . . . . . . . . 124 


\section{List of Figures}

1.1 British Skynet Satellite System . . . . . . . . . . . . . . 2

1.2 LEO Debris . . . . . . . . . . . . . . . . . . . 5

1.3 Chelyabinsk Bolide . . . . . . . . . . . . . . 6

1.4 PAM-D Re-entry . . . . . . . . . . . . . . 8

2.1 Asteroid Disruption Modeling at LANL . . . . . . . . . . . . . . 11

2.2 Asteroid Disruption Methods . . . . . . . . . . . . . . . 13

2.3 Monolithic Shield . . . . . . . . . . . . . . . . . . . . . . . 14

3.1 Framework Modules . . . . . . . . . . . . . . . . 20

3.2 Fragment Power Distribution . . . . . . . . . . . . . . . . . 24

3.3 Results From Hydrodynamic Code . . . . . . . . . . . . . . . . 27

3.4 Fitting Distributions - Wie1 . . . . . . . . . . . . 27

3.5 Fitting Distributions - Wiela . . . . . . . . . . . . . 28

3.6 Fitting Distributions - Wie1b . . . . . . . . . . . . . . . 28

3.7 Wie1 Cumulative Distribution Function . . . . . . . . . . . . . . 29

3.8 Wie1 Inverse CDF . . . . . . . . . . . . . . . . . . . . 29

3.9 Wie1 Distribution Function Rebuilt . . . . . . . . . . . . . . . . 30

3.10 Weaver1 Original Distribution . . . . . . . . . . . . . . 31 
3.11 Weaver1 Logistic Fit . . . . . . . . . . . . . . . . . . . 31

3.12 Weaver1 Inverse CDF . . . . . . . . . . . . . . . . . . . 31

3.13 GEO Golden Section Search . . . . . . . . . . . . . . . . . 39

3.14 Poisson Distribution by Fragment Size . . . . . . . . . . . . 44

4.1 Moon Residual . . . . . . . . . . . . . . . . . . . . . . 54

4.2 Earth Residual $(\mathrm{km}) \ldots \ldots \ldots \ldots$. . . . . . . . . . 55

4.3 Earth Residual (AU) . . . . . . . . . . . . . . . . . . . 55

4.4 Apophis Residual 1yr . . . . . . . . . . . . . . . . . . 56

4.5 Apophis Residual $2 \mathrm{yr} \ldots \ldots \ldots \ldots$

4.6 Apophis Residual 30 Days . . . . . . . . . . . . . . . . 57

4.7 Weaver2 Original Distribution . . . . . . . . . . . . . . . 59

4.8 Weaver2 Simulated Distribution . . . . . . . . . . . . . . . . . . 59

5.1 LEO425 4/9/2029 Text . . . . . . . . . . . . . . . . . . . . . 67

5.2 LEO425 4/9/2029 MMOD Mean . . . . . . . . . . . . . . 68

5.3 LEO425 4/9/2029 MMOD CI . . . . . . . . . . . . . . . . 68

5.4 LEO425 4/9/2029 Orbit Check . . . . . . . . . . . . . . . . 70

5.5 LEO425 4/9/2029 Longitude Bins . . . . . . . . . . . . . . . 70

5.6 LEO425 4/9/2029 Heatmap . . . . . . . . . . . . . . . . . . . . 71

5.7 GEO1 4/9/2029 Text . . . . . . . . . . . . . . . . . . 72

5.8 GEO1 4/9/2029 MMOD Mean . . . . . . . . . . . . . . . . . . . 73

5.9 GEO1 4/9/2029 MMOD CI . . . . . . . . . . . . . . . 73

5.10 GEO1 4/9/2029 Orbit Check … . . . . . . . . . . 73

5.11 GEO1 4/9/2029 Longitude Bins . . . . . . . . . . . . . . . 73

5.12 GEO1 4/9/2029 Heatmap w/Satellites . . . . . . . . . . . . 73

8.1 LEO800 4/9/2029 Text . . . . . . . . . . . . . . . . . . . . . 88

8.2 LEO800 4/9/2029 MMOD Mean . . . . . . . . . . . . . . . 88 
8.3 LEO800 4/9/2029 MMOD CI . . . . . . . . . . . . . . . . 88

8.4 LEO800 4/9/2029 Orbit Check . . . . . . . . . . . . . . . . 89

8.5 LEO800 4/9/2029 Longitude Bins . . . . . . . . . . . . . . . . . . 89

8.6 LEO800 4/9/2029 Heatmap . . . . . . . . . . . . . . . . . . . . 89

8.7 LEO1200 4/9/2029 Text . . . . . . . . . . . . . . . . . . 90

8.8 LEO1200 4/9/2029 MMOD Mean . . . . . . . . . . . . . . . . . 90

8.9 LEO1200 4/9/2029 MMOD CI . . . . . . . . . . . . . . . . . 90

8.10 LEO1200 4/9/2029 Orbit Check . . . . . . . . . . . . . . . . 91

8.11 LEO1200 4/9/2029 Longitude Bins . . . . . . . . . . . . . . . 91

8.12 LEO1200 4/9/2029 Heatmap . . . . . . . . . . . . . . . . . . 91

8.13 GEO4 4/9/2029 Text . . . . . . . . . . . . . . . . . . 92

8.14 GEO4 4/9/2029 MMOD Mean . . . . . . . . . . . . . . . . . 92

8.15 GEO4 4/9/2029 MMOD CI . . . . . . . . . . . . . . . . 92

8.16 GEO4 4/9/2029 Orbit Check . . . . . . . . . . . . . . . . 93

8.17 GEO4 4/9/2029 Longitude Bins . . . . . . . . . . . . . . . . . . 93

8.18 GEO4 4/9/2029 Heatmap . . . . . . . . . . . . . . . . . . 93

8.19 GEO15 4/9/2029 Text . . . . . . . . . . . . . . . . . . 94

8.20 GEO15 4/9/2029 MMOD Mean . . . . . . . . . . . . . . . . . 94

8.21 GEO15 4/9/2029 MMOD CI . . . . . . . . . . . . . . . . . . 94

8.22 GEO15 4/9/2029 Orbit Check . . . . . . . . . . . . . . . . . . 95

8.23 GEO15 4/9/2029 Longitude Bins . . . . . . . . . . . . . . . . 95

8.24 GEO15 4/9/2029 Heatmap . . . . . . . . . . . . . . . . . . 95

8.25 LEO425 4/11/2029 Text . . . . . . . . . . . . . . . . 96

8.26 LEO425 4/11/2029 MMOD Mean . . . . . . . . . . . . . . . . . 96

8.27 LEO425 4/11/2029 MMOD CI . . . . . . . . . . . . . . . . . . . 96

8.28 LEO425 4/11/2029 Orbit Check . . . . . . . . . . . . . . . . . . 97

8.29 LEO425 4/11/2029 Longitude Bins . . . . . . . . . . . . . . . . . . 97 
8.30 LEO425 4/11/2029 Heatmap . . . . . . . . . . . . . . . . . . . . 97

8.31 LEO800 4/11/2029 Text . . . . . . . . . . . . . . . . . . . . 98

8.32 LEO800 4/11/2029 MMOD Mean . . . . . . . . . . . . . . . . 98

8.33 LEO800 4/11/2029 MMOD CI . . . . . . . . . . . . . . . . . . . 98

8.34 LEO800 4/11/2029 Orbit Check . . . . . . . . . . . . . . . . . 99

8.35 LEO800 4/11/2029 Longitude Bins . . . . . . . . . . . . . . . . . . . 99

8.36 LEO800 4/11/2029 Heatmap . . . . . . . . . . . . . . . . . . . . 99

8.37 LEO1200 4/11/2029 Text . . . . . . . . . . . . . . . . 100

8.38 LEO1200 4/11/2029 MMOD Mean . . . . . . . . . . . . . . . . 100

8.39 LEO1200 4/11/2029 MMOD CI . . . . . . . . . . . . . . . . 100

8.40 LEO1200 4/11/2029 Orbit Check . . . . . . . . . . . . . . . . 101

8.41 LEO1200 4/11/2029 Longitude Bins . . . . . . . . . . . . . . . . 101

8.42 LEO1200 4/11/2029 Heatmap . . . . . . . . . . . . . . . . . . . 101

8.43 GEO1 4/11/2029 Text . . . . . . . . . . . . . . . . . 102

8.44 GEO1 4/11/2029 MMOD Mean . . . . . . . . . . . . . . . 102

8.45 GEO1 4/11/2029 MMOD CI . . . . . . . . . . . . . . . . . . 102

8.46 GEO1 4/11/2029 Orbit Check . . . . . . . . . . . . . . . . . . 103

8.47 GEO1 4/11/2029 Longitude Bins . . . . . . . . . . . . . . . 103

8.48 GEO1 4/11/2029 Heatmap . . . . . . . . . . . . . . . . . 103

8.49 GEO4 4/11/2029 Text . . . . . . . . . . . . . . . . . 104

8.50 GEO4 4/11/2029 MMOD Mean . . . . . . . . . . . . . . . . . . 104

8.51 GEO4 4/11/2029 MMOD CI . . . . . . . . . . . . . . . 104

8.52 GEO4 4/11/2029 Orbit Check . . . . . . . . . . . . . . . . 105

8.53 GEO4 4/11/2029 Longitude Bins . . . . . . . . . . . . . . . . . 105

8.54 GEO4 4/11/2029 Heatmap . . . . . . . . . . . . . . . 105

8.55 GEO15 4/11/2029 Text . . . . . . . . . . . . . . 106

8.56 GEO15 4/11/2029 MMOD Mean . . . . . . . . . . . . 106 
8.57 GEO15 4/11/2029 MMOD CI … . . . . . . . . . . 106

8.58 GEO15 4/11/2029 Orbit Check ． . . . . . . . . . . . . . 107

8.59 GEO15 4/11/2029 Longitude Bins . . . . . . . . . . . . . . . . 107

8.60 GEO15 4/11/2029 Heatmap . . . . . . . . . . . . . . . . . . 107

8.61 LEO425 4/9/2029 Text . . . . . . . . . . . . . . . . . . . . . 108

8.62 LEO425 4/9/2029 MMOD Mean . . . . . . . . . . . . . . . . . 108

8.63 LEO425 4/9/2029 MMOD CI . . . . . . . . . . . . . . . . 108

8.64 LEO425 4/9/2029 Orbit Check . . . . . . . . . . . . . . . . . 109

8.65 LEO425 4/9/2029 Longitude Bins . . . . . . . . . . . . . . . . . 109

8.66 LEO425 4/9/2029 Heatmap . . . . . . . . . . . . . . . . . . . . . 109

8.67 GEO1 4/9/2029 Text . . . . . . . . . . . . . . . . . . 110

8.68 GEO1 4/9/2029 MMOD Mean . . . . . . . . . . . . . . . . . . 110

8.69 GEO1 4/9/2029 MMOD CI ～. . . . . . . . . . . . . . . . 110

8.70 GEO1 4/9/2029 Orbit Check . . . . . . . . . . . . . . . . 111

8.71 GEO1 4/9/2029 Longitude Bins . . . . . . . . . . . . . . . . . 111

8.72 GEO1 4/9/2029 Heatmap . . . . . . . . . . . . . . . . . . . . 111

8.73 LEO425 4/9/2029 Text . . . . . . . . . . . . . . . . . . . . . . 112

8.74 LEO425 4/9/2029 MMOD Mean . . . . . . . . . . . . . . . . 112

8.75 LEO425 4/9/2029 MMOD CI … . . . . . . . . . . . . . 112

8.76 LEO425 4/9/2029 Orbit Check . . . . . . . . . . . . . . . . 113

8.77 LEO425 4/9/2029 Longitude Bins . . . . . . . . . . . . . . . . . 113

8.78 LEO425 4/9/2029 Heatmap . . . . . . . . . . . . . . . . . . . . 113

8.79 GEO1 4/9/2029 Text . . . . . . . . . . . . . . . . . . . 114

8.80 GEO1 4/9/2029 MMOD Mean . . . . . . . . . . . . . . . . . . 114

8.81 GEO1 4/9/2029 MMOD CI … . . . . . . . . . . . . . 114

8.82 GEO1 4/9/2029 Orbit Check . . . . . . . . . . . . . . 115

8.83 GEO1 4/9/2029 Longitude Bins . . . . . . . . . . . . . . . . 115

xiv 
8.84 GEO1 4/9/2029 Heatmap . . . . . . . . . . . . . . . . 115 


\section{Chapter 1}

\section{Introduction}

Micrometeoroids and orbital debris (MMOD) is a growing issue with international importance. Micrometeoroids are naturally occurring fragments of rock and dusk that exist throughout the solar system. Orbital debris is human made material like rocket bodies, paint flakes, and the effluent of spacecraft collisions. Even small MMOD particles on the order of $1 \mathrm{~cm}$ in diameter have the potential to destroy critical spacecraft systems. Because of this, MMOD is a threat to all spacecraft in orbit. Even governments that most sternly oppose US international policy have a stake when it comes to minimizing MMOD flux. Space-based assets are essential to support the growing demand for high-capacity communications networks around the world. These networks support services that civilian and military users have grown accustomed to using on a daily basis: Global Positioning System (GPS), Satellite Radio, Internet Backhaul, Unmanned Areal Vehicles (UAVs), and Reconnaissance Satellites [Figure 1.1]. A sudden loss of these services could degrade the 
warfighter's capabilities and cripple commercial enterprises that rely on these technologies. Manned space efforts like the International Space Station (ISS) could also suffer as a result of increased MMOD flux.

\section{SKYNET COMMUNICATIONS}

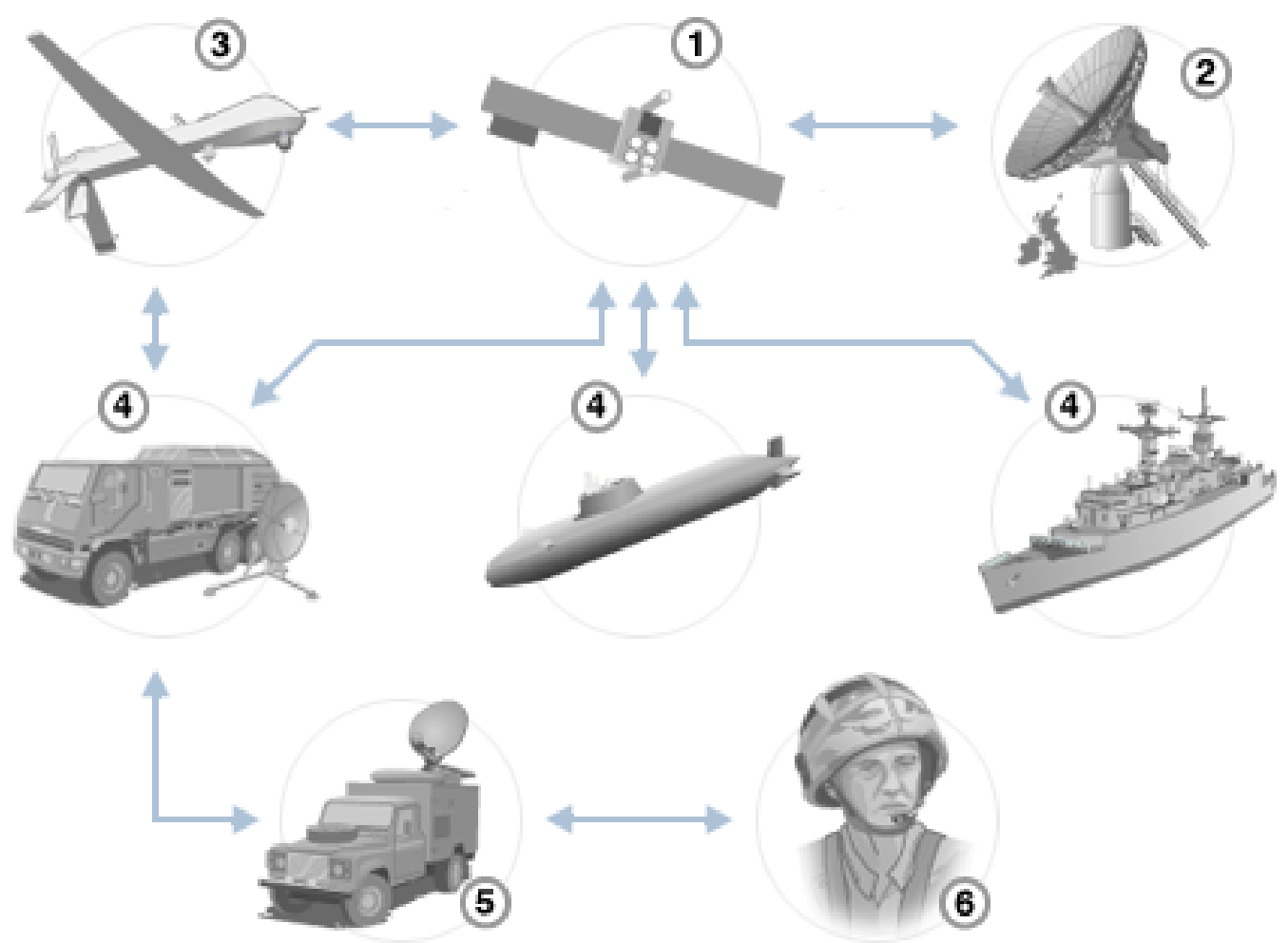

Figure 1.1: British Skynet System relies on Satellites for Communications Backbone[5]

Spacecraft have been damaged due to being hit by MMOD. The optical telescope on the Solar A spacecraft lost functionality after its sun shade was punctured by MMOD. During the Perseid meteor shower, Olympus 1 lost its lock on the sun due to a MMOD related event that. The satellite then exhausted all of its fuel trying to 
relocate the sun [25]. The loss of this satellite cost the US government $\$ 850$ million dollars [13]. Years later, Landsat 5 also was damaged during Persieds and spun out of control.

In a recent interview in Aerospace America, Martin Faga, former assistant secretary of the Air Force for Space and former director of the NRO summed up the importance of satellites:

Satellites are so important to us now. They're the nervous system of our military today, and if the right nerves are blocked, even temporarily, basically the whole network can be taken down. Which is to say again that an adversary might get to the satellite by electronic means and never have to do anything physical, like hitting it with a kinetic kill vehicle.[10]

Asteroid disruption has the potential to create billions of kinetic kill vehicles.

Despite the realization the orbital debris is a growing problem, many nations have performed and continue to perform anti-satellite tests. These tests typically involve the catastrophic disruption satellites through kinetic impactors. China conducted an anti-satellite test in 2007. The target was the Feng Yun 1C polar orbit weather satellite. The satellite was orbiting at an approximate altitude of $865 \mathrm{~km}$ when it was struck by a Chinese ground-launched missile [1]. The United States conducted an anti-satellite test in 2008. Using a ship-launched SM3 missile (made by the Raytheon company), the satellite US 193 was successfully targeted and destroyed shortly before reentry into Earth's atmosphere. The approximate height of the satellite at intercept was reported to be $241 \mathrm{~km}$ [3]. Previous testing by the US and Russia stretch back to the 1960s. The debris from the Chinese test, as well as other high-altitude tests, will persist in LEO for decades. Orbital debris from tests at lower altitudes re-enter 
the atmosphere quickly and create little persistent debris. Thus, after the Chinese test there was considerable international outcry.

Spacecraft are increasingly put in jeopardy by MMOD. The majority of debris in LEO is predominantly human-made orbital debris. These debris are assumed to have densities close to aluminum $\left(2.8 \mathrm{~g} / \mathrm{cm}^{3}\right)$ and can cause serious damage when they impact a spacecraft. The relative velocity on impact for these objects is estimated to be around 1 to $3 \mathrm{~km} / \mathrm{s}$ in LEO. The near-Earth space environment is rapidly filling up with orbital debris due to the hot pace of current satellite launches and an increasing number of old satellites that have not been deorbited [Figure 1.2]. There is growing concern that a Kessler Syndrome may arise. Kessler theorized that with enough debris in orbit, it would disable other satellites and create more debris, creating a chain reaction [12]. This type of effect could ultimately lead to the long-term denial of access to critical orbit regimes for all nations [22].

Micrometeoroids by contrast, can have densities ranging from 0.5 to $2 \mathrm{~g} / \mathrm{cm}^{3}$ (ice) up to $8 \mathrm{~g} / \mathrm{cm}^{3}$ (iron). Typical impact velocities are upwards of $70 \mathrm{~km} / \mathrm{s}$ [25]. While micrometeoroids are fewer in number, especially in LEO orbits, they make up for this by packing a much larger punch than OD particles. Since the energy of a particle is related to $m * V^{2}$, the energy increases as a square of the velocity. Thus lighter particles at a higher velocity can be more dangerous to spacecraft than heavier particles at lower velocities.

Compounded with these current MMOD problems, disrupting an asteroid on an impact course with Earth will create a new source of MMOD. Disrupting consists 


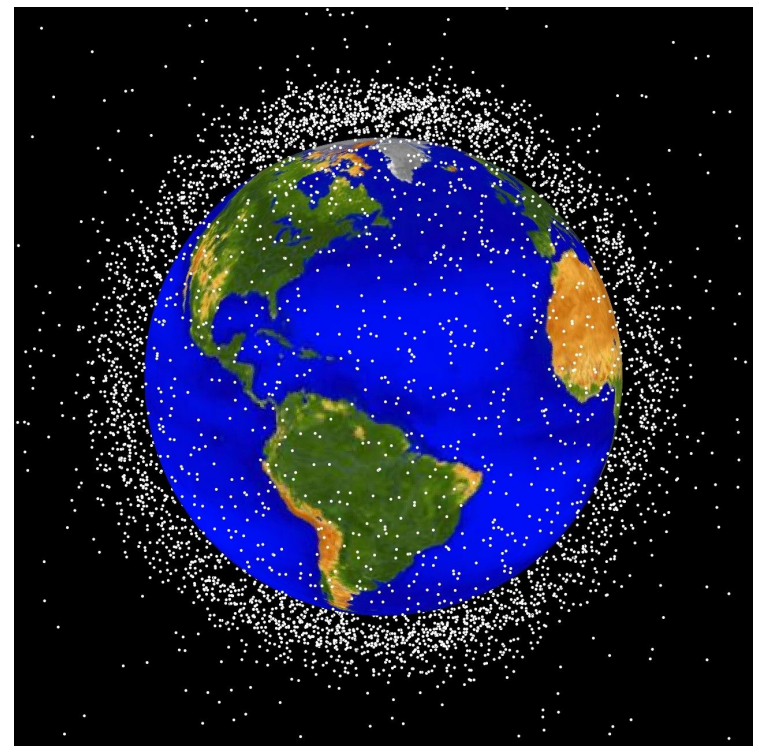

Figure 1.2: Orbital Debris in LEO Shown as White Dots (not to scale) [32]

of physically destroying the asteroid. This contrasts with deflection which typically involves non-destructive technologies to alter the orbit of the asteroid. Asteroid threat mitigation covered both deflection and disruption.

On November 9th, 2011, the asteroid YU55 passed within 0.85 Earth-Moon radii. This type of close approach by an asteroid capable of creating a nation-ending explosion if it hit earth is concerning. More recently, on February 14th 2013, a previously undetected asteroid exploded over the Russian city of Chelyabinsk [19] [Figure 1.3]. Estimates from the Russian Academy of Sciences suggest the object it released the equivalent of $500 \mathrm{kT}$ of TNT. However, the size of the object was only around 15 $\mathrm{m}$ in diameter with a weight of 10,000 tons. That is about the size and weight of a loaded tractor trailer. The asteroid was caught on tape and video and exploded in a 
fireball (a bolide).

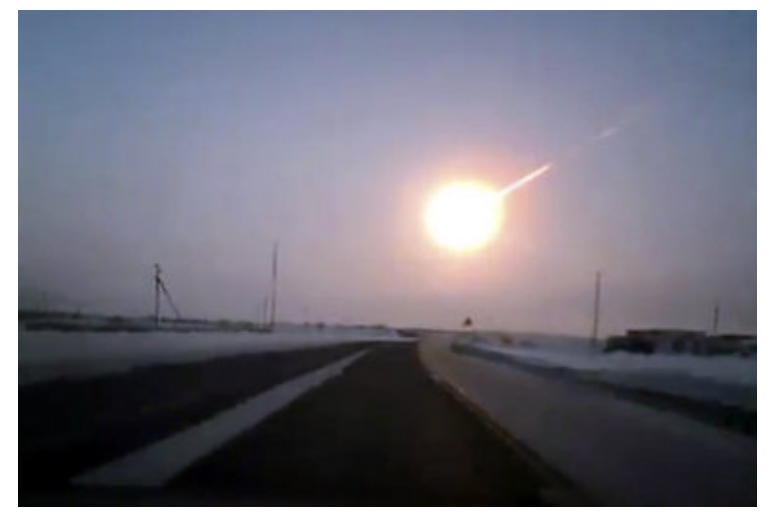

Figure 1.3: An asteroid burning up near Chelyabinsk 2013 [29]

The resulting shock wave blew out windows through Chelyabinsk, blew the roof off a Zink factory, damaged 3,000 buildings, and caused widespread injuries. The majority of injuries were minor and associated with broken glass. While events like those at Chelyabinsk are expected to occur once every 100 years [9], as the world becomes more populated, there is an increasing chance that serious damages to property and people will occur.

NASA has so far succeeded in documenting $90 \%$ of asteroids larger than $1 \mathrm{~km}$ diameter and is working towards the goal of $90 \%$ of all asteroids $140 \mathrm{~m}$ or larger by 2020. Although, most experts agree that without additional funding, this target will not be met. NASA spends only around $\$ 6$ million annually on ground-based NEO surveys and research [18]. After the Chelyabinsk event, NASA reacted by increasing funding four fold. However, funding has yet to be allocated to a space-based search program. Ground based detectors cannot detect asteroids coming at us from the 
direction of the sun. The only method to spot these threads is to station a satellite equipped with infra-red detectors in a trailing Earth or Venus orbit. This will allow the satellite to look away from the Sun in the direction of Earth, and detect asteroids headed towards us. Current ground-based search programs may reveal Near Earth Asteroids that have a non-negligible probability of hitting Earth. Additionally, with the margin of error in current orbital determinations, there is a chance that asteroids that have already been discovered will impact with Earth in future decades.

The international astronautic community is divided as to how large the asteroid has to be before it requires mitigation. The critical size that requires mitigation depends primarily on atmosphere's capability to disintegrate the incoming asteroid. Disintegration is a function of the size of the asteroid and the internal composition. Stony chondrites made of loose-amalgamations of matter may be easily disintegrated. However, it should be noted that metallic materials are not so easily consumed upon atmospheric entry. Titanium fuel tanks from satellites have successfully survived re-entry and have been found in farms and fields [Figure 1.4]. Generally speaking, asteroids of 30 meters or larger with metallic cores may need to be deflected.

The side effects of asteroid disruption are not well characterized which presents unknown risks if a disruption is required. In order to completely understand the consequences to ground and space-based resources, systems to evaluate these unknown risks must be created. The work presented here focuses on characterizing the potential change in MMOD fluence in critical orbit regimes due to nuclear surface and subsurface asteroid disruption. To accomplish this, multidisciplinary framework is required. This framework will calculate the transient MMOD fluence from a variety 


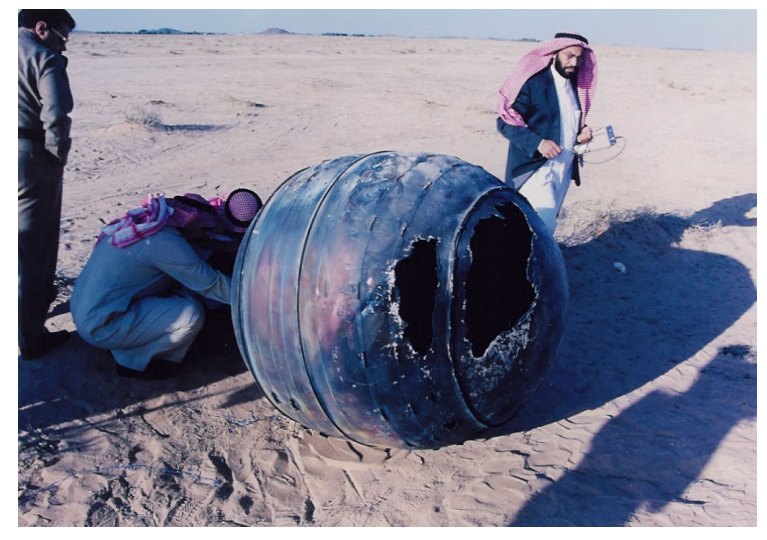

Figure 1.4: January 2001, PAM-D titanium motor casing survives re-entry and lands in Saudi Arabia. (Photo courtesy of NASA)

of scenarios. Several example disruption scenarios will be presented and evaluated. This will allow for the characterization of the side effects of destroying a asteroids and the risks to satellites in critical orbit regimes. 


\section{Chapter 2}

\section{Previous Work}

Asteroid disruption can be considered a nascent field where much of the work to date has been disruption modeling and trade studies of mitigation methods. The focus on disruption has often ignored the issue of side effects. These side effects are increasingly important when considerations for spacecraft shielding are taken into account. Shielding is designed only to withstand the typical MMOD flux. A review of flux models is provided. Finally, a review of the recent inter agency planning exercises is presented. These exercises have been conducted to understand the types of problems authorities will face in the case of a NEO impact event. 


\subsection{Fragmentation}

Test studies on fragmenting spherical alumina cement samples was conducted in 1998 by Giblin [16]. Their studies simulated asteroid to asteroid impact at $6 \mathrm{~km} / \mathrm{s}$ which could be an considered similar to a kinetic impactor. They produced fragment velocity curves and found that the ejection velocity field is fairly symmetrical about the impact direction. They also noted that there was a only a small correlation between fragment speed and size. For this analysis, the assumption is made that there is no correlation between fragment speed and $\delta \mathrm{V}$ imparted to the fragment by disruption. There is much other work in fragmentation. However, for this study, the concepts outlined by Giblin will suffice [16].

\subsection{Disruption Modeling}

Modeling the nuclear disruption of asteroids was embraced by researchers at Lawrence Livermore (LLNL) and Los Alamos National Labs (LANL). These laboratories have been tasked with ensuring that the nation's nuclear weapons are still fully operational despite the nuclear Test Ban treaty of 1963. This treaty prevents the US from conducting test detonations of nuclear weapons. Dr. David Dearborn began applying hydrodynamics simulations to the problems of asteroid disruption in 2007 [15]. Over subsequent iterations of this work, he partnered with Dr. Bong Wie and Brian Kaplinger at Iowa State University. The trio produced additional hydrodynamics simulations which concentrated on examining the dispersal patter of the 
asteroid Apophis [20][21]. Research by Wie and Dearborn has focused on plotting large particles tens of meters in diameter and determining if they impacted or missed earth. They did not account for smaller $1 \mathrm{~cm}$ to $10 \mathrm{~cm}$ sized objects. Objects in this range are still hazardous to satellites because orbital debris shielding is unable to withstand impact from these sized projectiles. Additionally, Dr. Robert Weaver at LANL has been modeling asteroid disruption using the Cielo supercomputer and the RAGE hydrodynamic modeling program [Figure 2.1]. The author partnered with Dr. Weaver to integrate the data from his studies as an input parameter of this study.

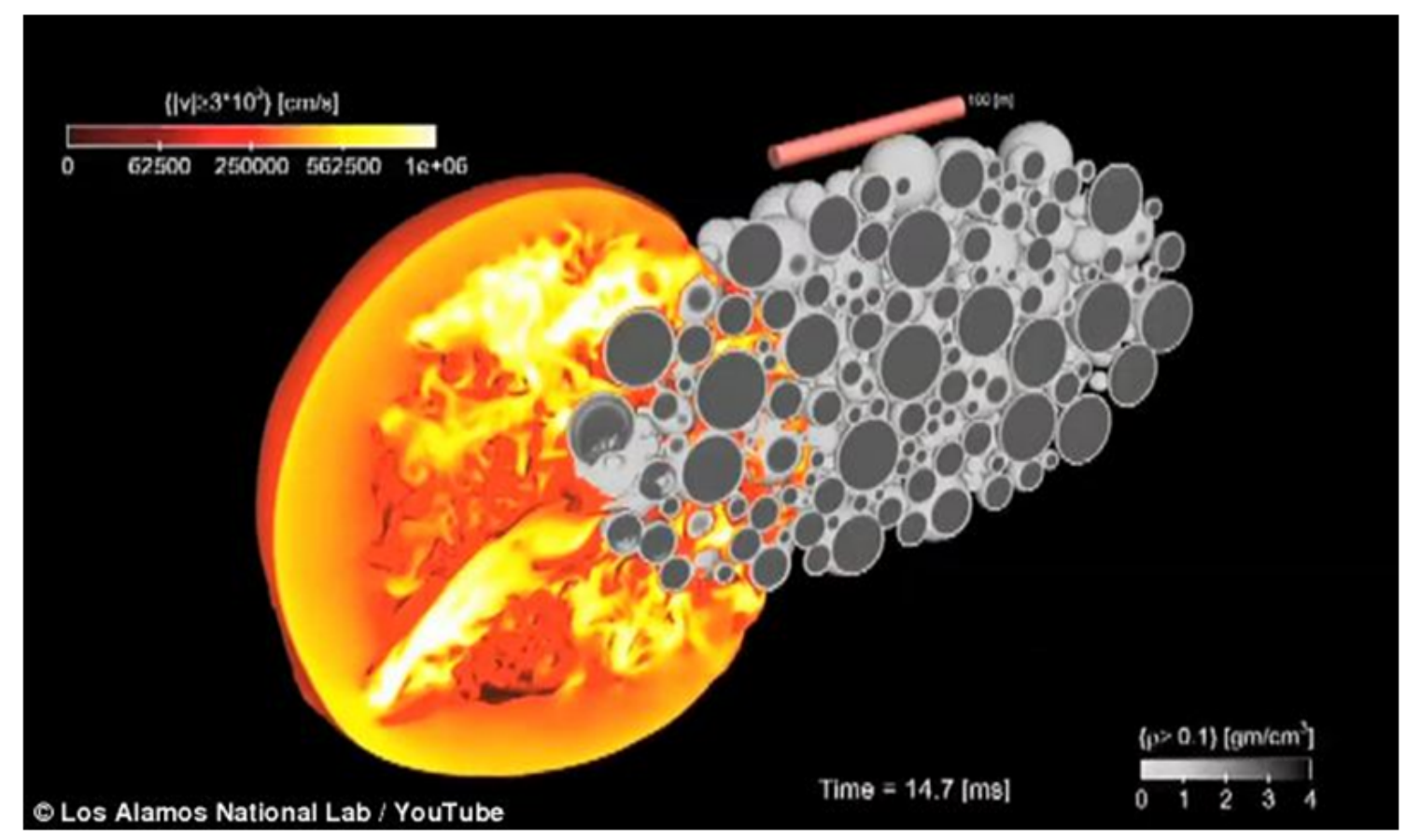

Figure 2.1: Asteroid Disruption Modeling at Los Alamos National Labs[35]. An Itokawa type asteroid deflected with a 1 megaton nuclear explosion. 


\subsection{Trade Studies}

Trade studies on asteroid mitigation are most clearly outlined by NASA's Report to Congress from 2007 [24] [Figure 2.2]. Bong Wie also presents a comprehensive outline of different mitigation techniques [36]. Methods of disrupting an asteroid include: Conventional Explosive (TNT) surface \& subsurface explosion, Kinetic surface \& subsurface impactor, Nuclear standoff explosion, and Nuclear surface \& subsurface explosion. Methods of deflecting an asteroid include: Focused solar energy, Pulsed laser, Mass driver, Gravity tractor, Tug, and Albedo Modification. Of all these methods, a nuclear explosion was found to be the most effective. This is because it could delivery the largest amount of energy to the asteroid without having to know anything about the surface or subsurface properties of the asteroid [24]. Addition-

ally, nuclear explosions are efficient in terms of the amount of energy delivered to the object compared to the cost of the mission and the probability of success [20].

Past studies on side effects of disruption have focused on Earth-impact events of ejected fragments [27]. Studies by Kaplinger, Wie, and Dearborn have shown that in some cases, Earth-impacting mass can be reduced by up to $90 \%$ through a combination of nuclear disruption and atmospheric burnup [21]. However, there are few mentions in literature on the expected effects that disruption could have on spacebased equipment. These include Gong, Ye and BaoLin 'The nuclear explosion may produce space nuclear pollution and may also generate some small fragmentations that threaten the Earth in another way and creates orbital debris on a solar system scale'[17]. While it is unclear what nuclear pollution may be, the orbital debris is a 


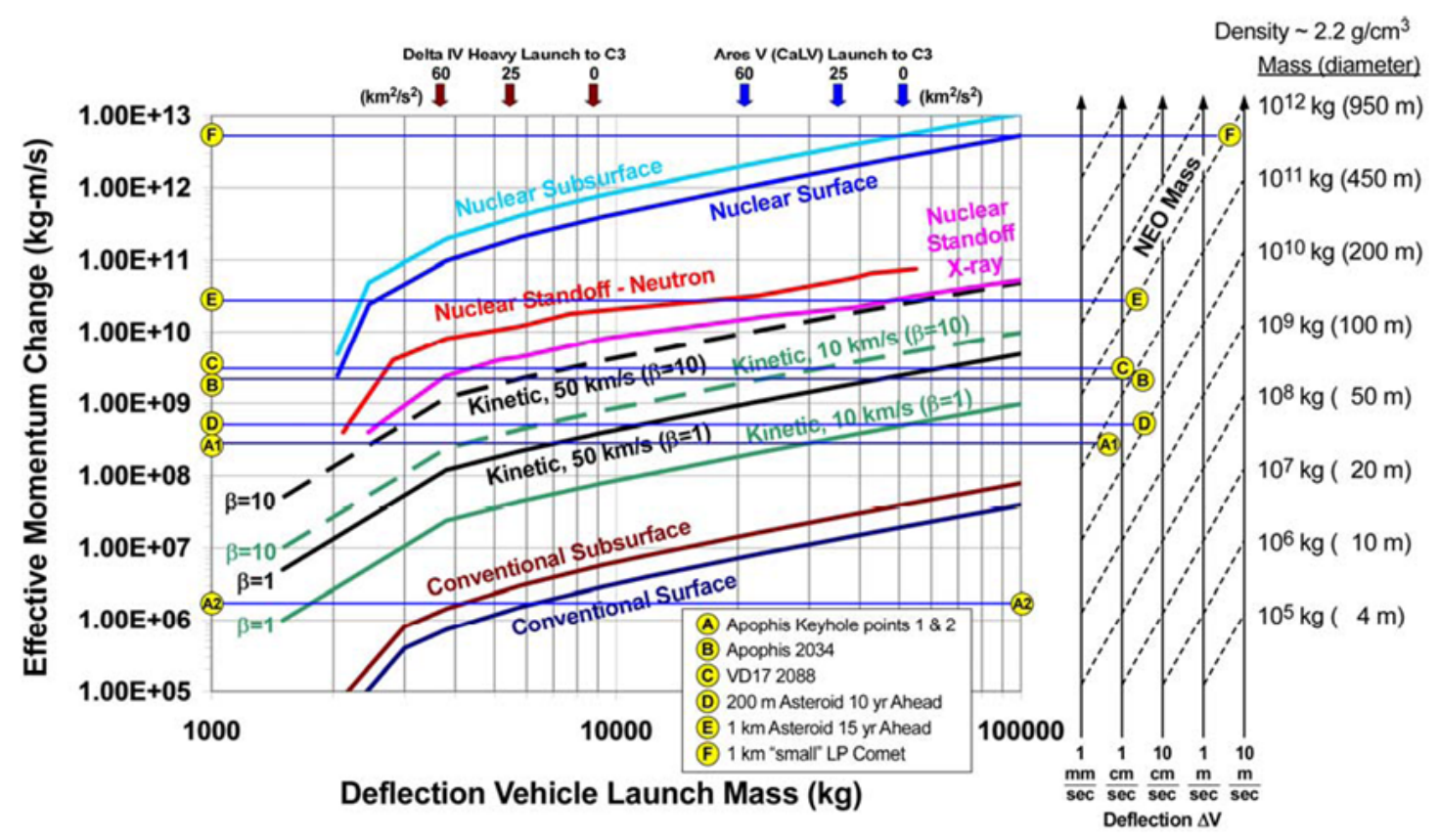

Figure 2.2: Asteroid Disruption Methods [24]

concern of theirs. Ahrens and Harris describe the debris coverage area in multiples of Earth-radii after a theoretical nuclear explosion. They concluded:

Thus fragmentation is likely to be a safe choice only for long lead-time response (decades) or for relatively small bodies where the fragments may still hit Earth. [4]

\subsection{Shielding}

Spacecraft shielding is essential to enable LEO spacecraft to survive the MMOD environment. At orbital velocities, particles the size of a pea travel through solid 
aluminum like butter [Figure 2.3]. Monolithic shields are particularly expensive and less effective than layered shielding. The Whipple and stuffed Whipple shield are typical types of shielding deployed on the international space station to protect ramfacing surfaces [26]. Research using the NASA Johnson Space Center Light Gas Gun has characterized the performance of these types of shields. The ram-facing surface of the spacecraft is the surface that receives the majority of MMOD strikes and is aligned with the velocity vector of the spacecraft. Typically, shielding is not deployed on other faces of the spacecraft. These types of shields are typically designed to mitigate $1 \mathrm{~cm}$ diameter or smaller particles. Each strike on the shield decreases the shield's subsequent effectiveness as the shield is partially destroyed around the impact location. While it is always possible to add more shielding, and thus survive larger particle impacts, the weight required quickly becomes prohibitive.

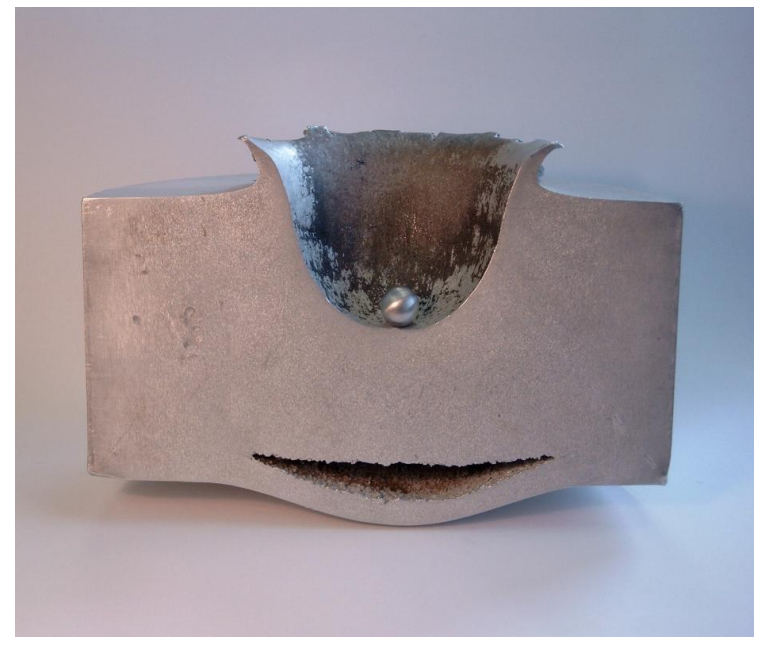

Figure 2.3: An Aluminum monolithic shield and test particle shows the penetration depth through similar materials at orbital velocities. (Image courtesy of NASA) 
Elements of the spacecraft that are not shielded typically include the solar arrays. On large GEO communications satellites, the surface area that the solar arrays take up can be in the hundreds of meters square. Additionally, the deployment of these arrays is typically omni-directional, once deployed, they cannot be retracted. Deployment devices have been designed to be on-way to save on weight but this does not lend to safety. If there is a larger MMOD fluence increase, even if the main body of the spacecraft is not struck, the solar arrays will most likely be impacted. Solar power is the life-blood of GEO communication satellites. The amount of power available dictates the number of signals and signal strength that can be transmitted back to Earth. A decrease in available power will decrease the satellites profitability and net worth.

\subsection{Flux Models}

Numerous codes exist for the assessment of Orbital Debris (OD). NASA Johnson Space Center has produced several Orbital Debris Engineering Models published in different years. To date, ORDEM96 and ORDEM2000 have been released. The newest version, ORDEM2010, also called ORDEM3.0 is still in the beta testing phase. ORDEM2000, which is available for download for the NASA orbital debris program office, charts the orbital debris flux up to $2000 \mathrm{~km}$ altitude. ORDEM3.0 is designed to be capable of handling debris up to $50,000 \mathrm{~km}$ altitude. This would be most useful for this study because it will allow the determination of expected orbital debris flux levels in GEO. However, these models do have limitations: 
one cannot evaluate the short-term collision risk, due to fragments from recent breakup events, relative to an orbiting satellite using an engineering model. [23]

The ORDEM models do not contain micrometeoroid components [25][12]. Details on NASA's development efforts and program capabilities are provided [11].

Micrometeoroid flux (MM) can be characterized in closed form by the Grun 1985 which is included in Tribble's recent publication [33]. The flux is considered omnidirectional and gravitational lensing and Earth shielding effects are included. For design considerations, a conservative estimate of the MM flux takes double the Grun results [25]. Assessment of the combined MMOD flux level can achieved by combining results for MM and OD analysis.

\subsection{Mitigation Planning}

The Natural Impact Hazard Inter Agency Deliberate Planning Exercise was conducted in 2008 by a wide conglomerate of elements from the US government and scientific advisory bodies [2]. Participants conducted a mock impending asteroid strike scenario. Organizations represented included: National Security Space Office, Department of Homeland Security (DHS), Department of State, National Aeronautics and Space Administration (NASA), Department of Energy (DOE), OSD Homeland Defense, National Military Command Center, Air Force Operations Group, Missile

Defense Agency, Defense Threat Reduction Agency, Air Force Checkmate \& Coast Guard, US Navy Strategic Concepts, Defense Threat Reduction Agency Weapons 
Effects, Lawrence Livermore National Lab (LLNL), Sandia National Lab, Air Force Research Laboratory Munitions (AFRL), Air Force Air Armaments Center, OSD Policy Planning, and Air Force Future Concepts. Highlights from their findings follow:

1) There is a deficit in software tools to support mitigation

None of our command centers have the necessary tools to make quick assessments. Players expressed a need for a National Decision Support System for natural impact scenarios and events. Such a system would tighten up the federated nature of impact prediction and impact effects prediction, integrating models for impact location \& uncertainty prediction, kinetic effects prediction, plume, and tsunami effects, and feed evacuation planning models... There is a corresponding need for an integrated suite of planning tools to allow end-to-end mission planning and decision support for deflection. Such a model should integrate astronautical navigation models, deflection modelling, launch windows, spacecraft \& launch vehicle production capabilities and schedule to clearly present to national leaders what options exist for deflection. [2]

2) Existing models do not capture side effects

Players highlighted the fact that current models inadequately address several effects likely to significantly affect accurate damage / effect estimates. These include the effect of blast plumes on Low Earth Orbit (LEO) satellites, electromagnetic effects that could affect electrical power infrastructure, seismic effects, effect of terrain on blast dissipation and focusing, coupling of air blast to tsunami response, and atmospheric distribution/dispersion of hazardous materials. [2]

3) The preferred approach for short-notice mitigation was stand-off nuclear

In this scenario, given the short lead time (less than a decade), players chose to go with a solution they felt was low mass, provided high energy 
density for deflection, leveraged existing national capabilities, and had comparatively high technological readiness level (TRL). ... A very different solution might be selected if there were multiple decades of warning, but then there would also be more time available to react after detection of the threat. The use of nuclear devices for this purpose would require significant international preparation or participation by other nuclear or space faring powers. [2]

These three conclusions frame the research done in this paper which seeks to create tools that otherwise do not exist. This will aid decision makers if faced with real an asteroid threat.

\subsection{Summary}

No asteroid mitigation missions have been conducted. And lacking the urgent need to deflect an asteroid, researchers have been unable to garner the support required to conduct basic research in this area. However, the threat posed by asteroid impacts and mitigation must be anticipated to avoid a knowledge gap should a threat arise. The technologies that enable asteroid mitigation take decades to implement. The analysis conducted in this thesis is geared towards making a turn-key solution to characterize one of the side effects of disruption an asteroid with a nuclear weapon. In this manner, this research supports basic disruption techniques. Additionally, this work supports expedient analysis and decision making to mitigate threats that disruption poses to space-based assets. This framework should be included in future decision-making software to aid in the overall mitigation effort planning. 


\section{Chapter 3}

\section{The Framework}

To assess the hazard posed to space assets but nuclear disruption of asteroids a framework was created o simulate the MMOD effluvia from such an event. The goal of the program was to be robust and modular to allow for the input of new information from future studies. The framework consists of six modules that act in concert to build a picture of the transient MMOD fluence increase. These six modules are: Input Parameters Module, Orbital Propagator Module, Orbit Crossing Counter Module, Statistical Analyzer Module, MMOD Fluence Analyzer Module, and Output Parameters Module [Figure 3.1].

The analysis start with information on the dispersion characteristics of asteroid hit with nuclear munitions (Input Parameters). The resulting debris field will be propagated forward in time using a Monte-Carlo method and an orbital simulator (Orbit Propagator). The number of particles that pass through critical orbit regimes 
Figure 3.1: The Six Modules of the Complete Framework to Evaluate MMOD Fluence from an Asteroid Disruption

will be assessed (Orbit Crossing Counter). Statistical methods are then applied to determine a sample size which will accurately reflect the characteristics of the fragment population (Statistical Analyzer). An estimate of the transient increase in MMOD fluence is made. The increase in fluence is compared to the typical MMOD levels to determine if there is a significant change (MMOD Fluence Analyzer). After processing all the data, a final assessment of the threat to space-based assets is given as well as other useful outputs which can help to further categorize the threat level. (Output Parameters). In this way, the program acts to efficiently simulate an asteroid dispersal.

The initial implementation was written in MATLAB R2011a. The files for the program are available for download as a zip file. Please contact Dr. Kira Abercromby in the Aerospace Engineering department at Cal Poly, SLO for details.

One important distinction that must be made is that this framework does not simulate what happens to an asteroid when it is hit with a nuclear explosion. That is a field of research being conducted by others at Iowa State, Lawrence Livermore National Labs, and Los Alamos National Labs (see Section 2). These types of sim- 
ulations usually involve hydrodynamics simulations that require supercomputers to complete. Instead, the final results from those simulations will be used as input into this framework. Additionally, this program only simulates one fragment of the asteroid at a time, after it has been hit with a nuclear weapon. This distinction will become obvious upon review of the input parameters 3.1.1.

\subsection{Overview of Modules}

What follows is a review of each individual module and its functionality and capabilities.

\subsubsection{Input Parameters}

The input parameters of the program are mean to be flexible. Some of the input is summary data from other studies. The option exists to use past studies, or to include analysis from outside as long as a few basic guidelines are followed.

Input parameters include: Asteroid Diameter, Largest Remaining Chunk, Disruption Date, Position of the Asteroid, Velocity of the Asteroid, The Position of Earth, The Velocity of Earth, and The Nuclear Explosion Profile.

Optional Inputs include: The observation date of the Position and Velocity of the asteroid and Earth, the number of fragments to create, as well as the complexity of the calculations which affects the number of additional planets included in the propagator. 
The program will prompt for the input parameters and if any additional parameters are desired.

\section{Asteroid Diameter}

The diameter of the asteroid in the units of meters is a required input parameter. At this time the program assumes a spherical asteroid for all volume calculations.

The actual diameter of asteroids is difficult to accurately determine unless it makes a close approach to earth or it is observed with spacecraft. Until January 9th, 2013, the asteroid Apophis was assumed to have a diameter of around $270+/-60 \mathrm{~m}$. However, during its close approach to earth in 2013, the Herschel Space Observatory got a good look at it and refined its diameter to $325+/-15 \mathrm{~m}$. This corresponds to a volume increase of $75 \%[8]$. This leads to a significant change in the total fragments expected from a nuclear disruption if one should occur.

For other less well known asteroids it is possible to get a rough estimate of the size by using the absolute magnitude (H) listed in the JPL small-body database browser which can be accessed at: http://ssd.jpl.nasa.gov/sbdb.cgi. One can then convert from a known absolute magnitude to diameter using Tables 8.4 and 8.5. Please note that this is based on an assumed 0.25 to 0.05 geometric albedo. Alternatively, if the albedo of the object is known, one can get a better estimate of the diameter of the asteroid by using Equation 3.1:

$$
\text { Diameter }=\frac{\left.1329 * 10^{(}-H / 5\right)}{\sqrt{\text { Albedo }}} * 1000
$$


where the resulting Diameter is in meters. $H$ is the absolute magnitude of the asteroid as given from the JPL database. Albedo is typically estimated at 0.154 unless a better estimate is known from direct observations.

\section{Largest Remaining Chunk}

An estimate of the Largest Remaining Chunk (LRC) or fragment of the asteroid after disruption in units of meters is a required input parameter. This is similar to saying: After the asteroid has been hit with a nuclear explosion, the largest piece of rock that is leftover has a diameter of $X$ meters. Where $X$ is the chunk size input into the program.

The primary purpose of this parameter is to allow for an estimate of the number of resulting particles from the explosion and the size of those particles. This type of characterization of the fragment size distribution is presented by Sanchez, Vasile and Radice [27]. They present an accumulative power law distribution to model

fragment size distribution. A one segment power law distribution is used in this study, however, two and three segment power laws have also been found to fit well to experimental data.

For the purposes of getting an initial estimate on the cumulative number of particles created by a disruption, Equations $3.2 \& 3.3$ will be used:

$$
N=m_{\max }^{b} * m^{-b}
$$




$$
b=\left(1+\frac{m_{\max }}{M_{a}}\right)^{-1}
$$

Where $N$ is the cumulative number of particles above a given mass size $m$. The mass of the largest remaining chunk is given by $m_{\max }$. The initial mass of the asteroid is $M_{a}$, with $b$ being an intermediate variable.

Using the asteroid Apophis as an example, there is a noticeable difference in cumulative number of particles, $N$, based on different assessments of $m_{\max }$.

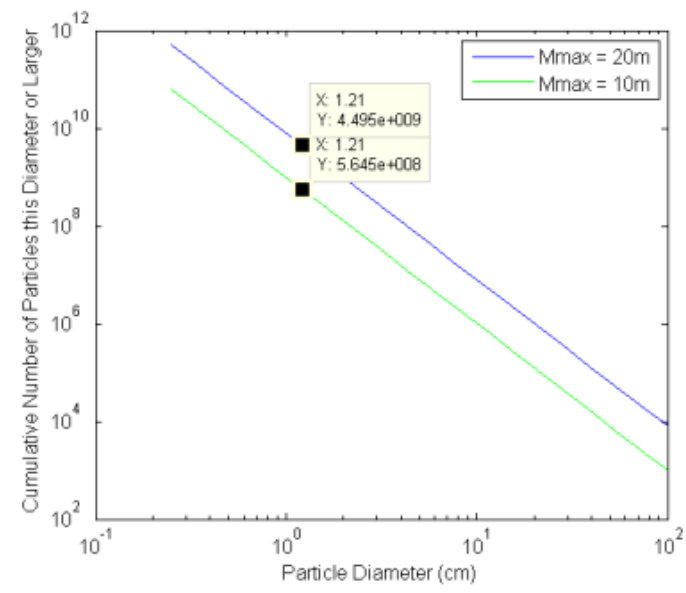

Figure 3.2: Fragment Power Distribution

Figure 3.2 shows that for an equivalent particle size, a disruption resulting with the largest remaining chunk of $20 \mathrm{~m}$ has almost ten times the number of particles with a diameter of $1.2 \mathrm{~cm}$ and larger. Shielding for spacecraft for particles above $1 \mathrm{~cm}$ in diameter is impractical as the amount of shielding mass required would consume all the mass required by the payload. Particles below $1 \mathrm{~cm}$ in diameter can be shielded for. Thus, to have the best chance of minimizing effects on space-based systems, 
it is critical to minimize the largest remaining chunk size and thus the number of particles of $1 \mathrm{~cm}$ diameter or larger resulting from the disruption.

Values of $m_{\max }$ are expected to be in the $20 \mathrm{~m}$ to $10 \mathrm{~m}$ range and are ultimately a function of the internal makeup of the asteroid in conjunction with the megaton yield of the nuclear explosion. If the largest fragment is around $10 \mathrm{~m}$ to $20 \mathrm{~m}$, atmospheric entry could destroy the particle. This would minimize the threat to ground-based resources. Thus it is assumed that for any disruption effort, the explosion will be sized to provide a largest remaining chunk in this diameter range.

\section{Disruption Date}

The date and time of the disruption is a required input parameter. This tells the program when the asteroid is hit by the nuclear explosion and will be the starting point for the individual fragment orbit propagations. The date should be in the J2000 reference frame and should be given as a vector with elements: [yyyy,month,day,hour,minute,second]. While it may be impossible to accurately know beforehand the exact minute and second of a disruption, estimating the day accurately is vitally important and can have a significant effect on MMOD fluence creation.

\section{Asteroid Position and Velocity}

Asteroid position and velocity are required input parameters. Both terms should be described in the Sun Centered Inertial (Heliocentric) reference frame. The helio- 
centric reference frame is aligned with the vernal equinox. This information is used by the propagator to move the asteroid forward or backward in time from some initial starting date to the point in its orbit at which it will be disrupted. The required format is a vector with elements: $[\mathrm{x}, \mathrm{y}, \mathrm{z}, \mathrm{Vx}, \mathrm{Vy}, \mathrm{Vz}]$. The position $(\mathrm{x}, \mathrm{y}, \mathrm{z})$ are in units of Astronomical Units (AU) and the velocity $(\mathrm{Vx}, \mathrm{Vy}, \mathrm{Vz})$ is in units of $\mathrm{AU} /$ day. This corresponds to typical units output by the JPL Ephemeris Database [31].

\section{Earth Position and Velocity}

Similarly, to the Asteroid Position and Velocity, the initial Earth Position (AU) and Velocity (AU/day) in the Heliocentric reference frame is required. Estimates of this can be obtained from the JPL Ephemeris Database [31]. The propagator uses this information to estimate the position of the Earth for each time step. The required format is a vector with elements: $[\mathrm{x}, \mathrm{y}, \mathrm{z}, \mathrm{Vx}, \mathrm{Vy}, \mathrm{Vz}]$.

\section{Explosion Profile}

The profile of the nuclear explosion used to deflect the asteroid is a required input. This profile describes the change in velocity of particles seconds after the explosion. This profile is included as an external MATLAB file. The file should have no inputs and only one output parameter which corresponds to the $\delta \mathrm{V}$ experienced by a particle in units of meters per second $(\mathrm{m} / \mathrm{s})$.

Several basic explosion profiles have been provided with the framework: Wie1, Weaver1, Weaver2, Weaver3, Weaver4, Weaver5. Each of these corresponds to a 
particular hydrodynamic simulation. For descriptions of each distribution see Section 8.4 .

One of the goals of the program is to make it simple to swap out the explosion profile. This will allow for many different scenarios to be tested other than the few provided in this paper. The explosion profile will naturally vary with the strength of the nuclear weapon used as well as the surface and subsurface characteristics of the asteroid in question.

To create the explosion profile, one must first simulate the nuclear weapon hitting the asteroid. This typically done with supercomputers and hydrodynamics code, and is well outside the scope of this paper. However, final velocity data supplied by these studies can be used as a starting point. Figure 3.3 shows the results of a hydrodynamics simulations from a disruption of the asteroid Apophis conducted by Wie [37]. Precise details on the fragmentation strength and model construction are available.

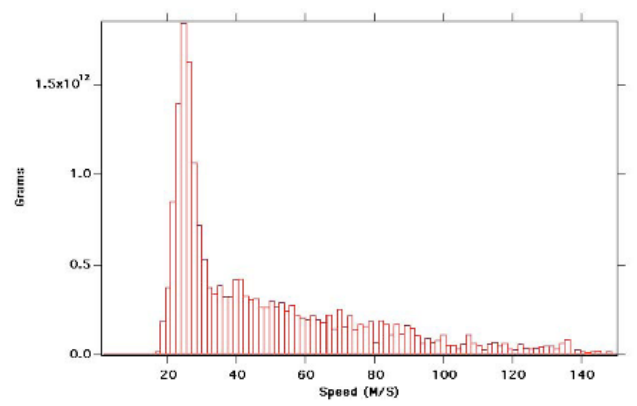

Figure 3.3: Speed Distribution, Apophis Fragmentation[37]

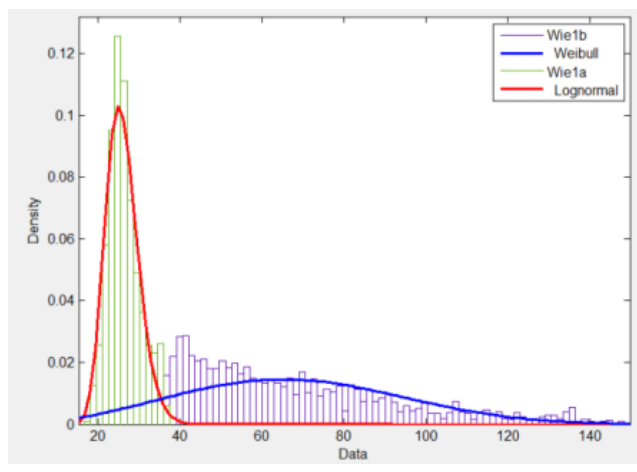

Figure 3.4: Wie1 Distribution in Two Parts 
The resulting distribution describes the change in velocity of the particles across the $\mathrm{x}$-axis and. the density of particles on the y-axis. Fitting this distribution to a single probability distribution is problematic. The data is split into two parts and fit separately [Figure 3.4]. The left side of the graph including the peak at approximately $30 \mathrm{~m} / \mathrm{s}$ was fit to a lognormal distribution Wiela [Figure 3.5]. With a p-value of 0.8, there is a possibility this data originated from a lognormal distribution. The other half of the distribution, Wie1b, can be fit to a Wiebull distribution [Figure 3.6]. However, the 0.098 p-value for this fit is much lower. Thus only $10 \%$ of the time would a Weibull distribution manifest in this distribution. However, since this is the best fit available, it will suffice. The fits below were created in Minitab 16.

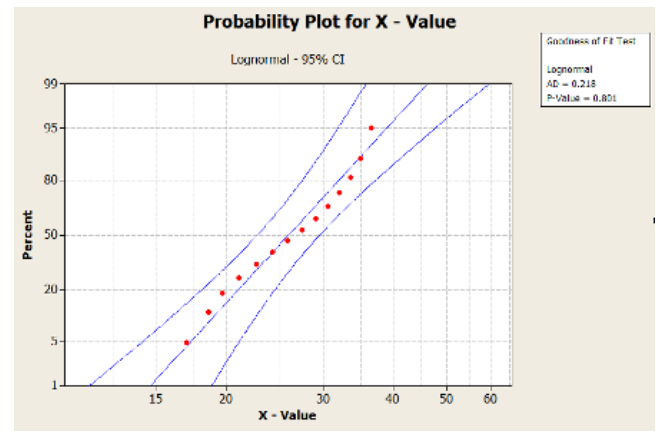

Figure 3.5: A hypothesis test for Lognormal Distribution

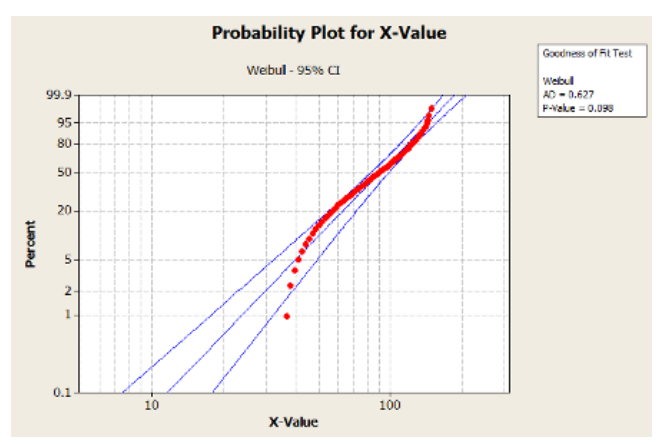

Figure 3.6: A hypothesis test for Weibull Distribution

A plot of the cumulative distribution function (CDF) of each of the distributions using MATLAB and built-in dfittool command is provided [Figure 3.7]. To find the inverse CDF, the individual CDFs are summed and the total is divided by two. This creates a graph where the total area under the curve is one. The inverse CDF does exist because there is only one $\mathrm{y}$ value to each $\mathrm{x}$ value. The CDF is inverted by 
placing 1000 points along the $\mathrm{x}$ axis and determining the corresponding $\mathrm{y}$ value. The data is mirrored about the $\mathrm{x}-\mathrm{y}$ plane to create the inverse CDF [Figure 3.8].

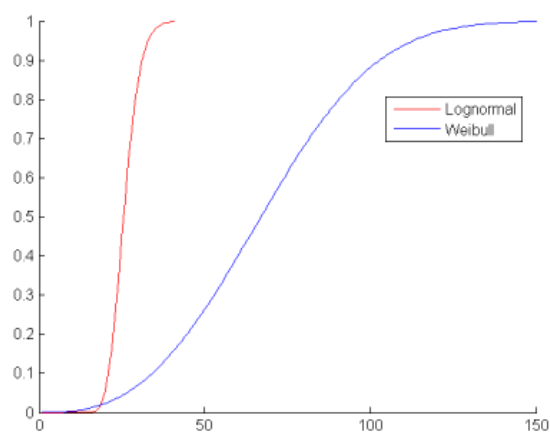

Figure 3.7: The Cumulative Distribution for Wie1 in Two Parts

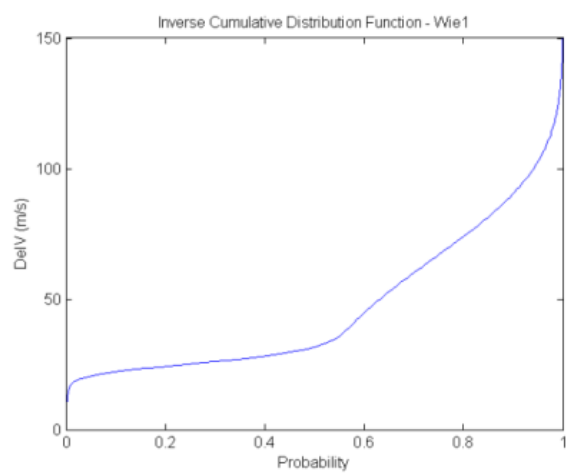

Figure 3.8: The Inverse of the Cumulative Distribution, Wie1

Using the matrix of corresponding $\mathrm{x}$ and $\mathrm{y}$ values, a random $\mathrm{x}$ value from $0-1$ is applied. Using the MATLAB spline command, a result for $\mathrm{y}$ is interpolated without having to use a closed form solution. The cumulative inverse CDF is useful because it allows points to be generated with the same velocity profile as the original $\delta \mathrm{V}$ curve. This is accomplished by applying a uniform distribution on the $\mathrm{x}$ axis. This will generate random points along the $\mathrm{x}$ axis. The resulting value y reflect the $\delta \mathrm{V}$ from the original distribution created by Wie. After running the Wie1.m file, 1000 times, this requests 1000 points in the distribution. A plot of the resulting explosion profile is shown in Figure 3.9. This profile is remarkably similar to the original Wie1 distribution. This is expected as one is derived from the other. 


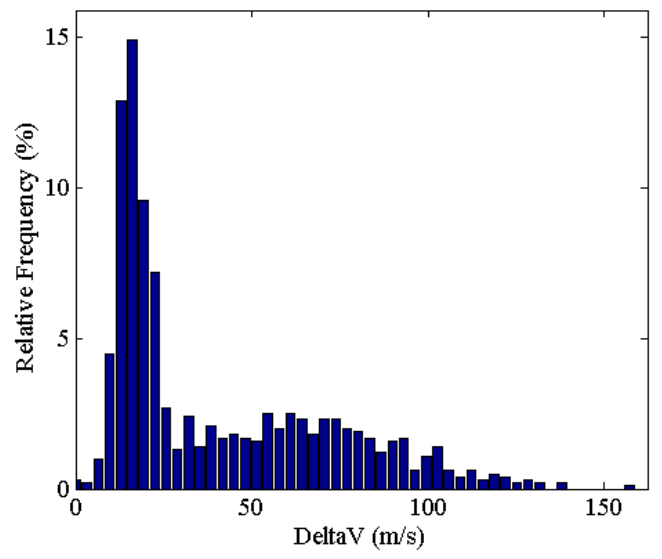

Figure 3.9: Similar to the Original Wie1 Distribution, Results from Running Wie1.m x1000

Distributions can be created by fitting a single function to the resulting velocity distribution. The Weaver1 profile is an example of this. It can be fitted using a single logistic distribution [Figure 3.10]. A logistic fit goes nicely with the Weaver1 distribution [Figure 3.11]. This allows us to directly transform the single CDF for this distribution into the inverse CDF. This inverse CDF function contained in the Weaver1.m file corresponds to Figure 3.12. 

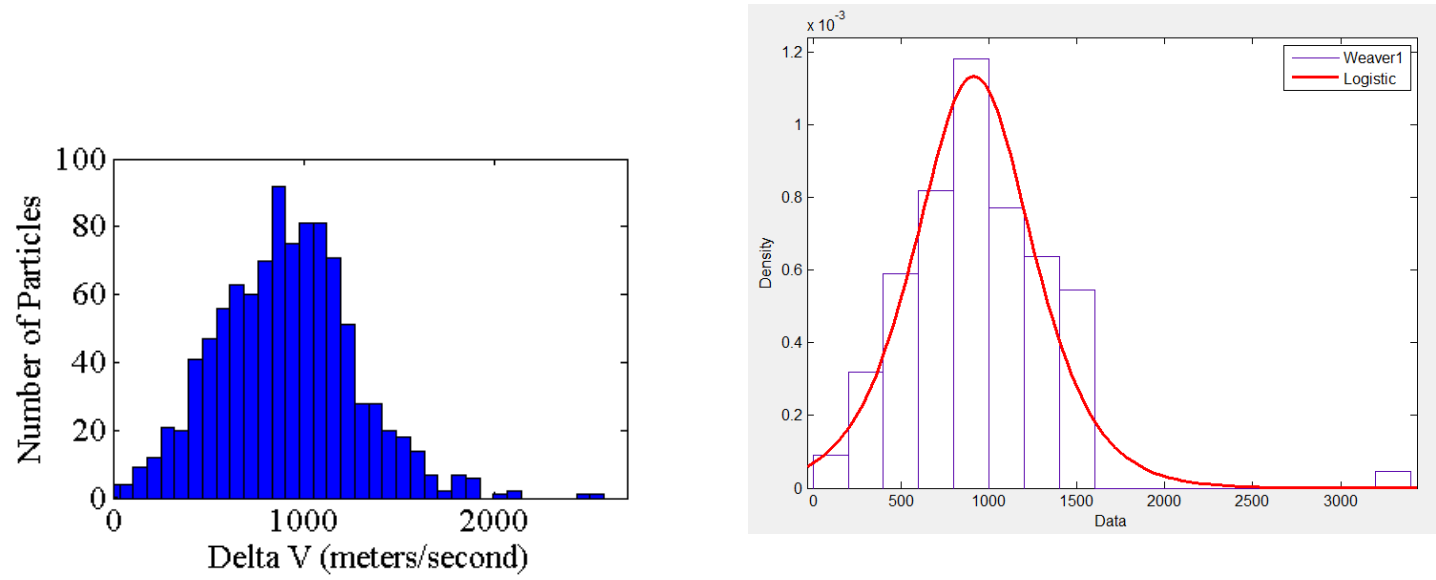

Figure 3.11: Logistic Fit,

Figure 3.10: Weaver1 Original Distribution

Weaver1 Distribution

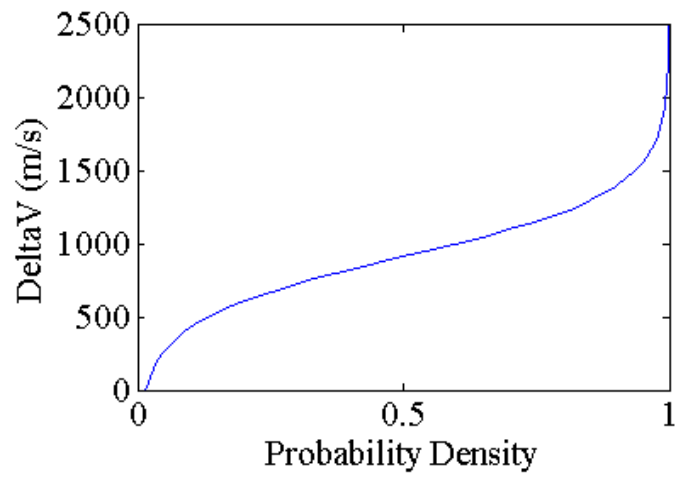

Figure 3.12: Weaver1 Inverse Cumulative Distribution Function (CDF)

\section{Optional Inputs}

Optional Inputs include the J2000 date at which the observations of the Earth's and Asteroid's position and velocity are made. This allows one to easily input starting 
information from the JPL ephemeris database at a given observation time, and then to study a range of later disruption times. If no parameter is provided, disruption date will be set as the observation date.

The number of fragments to run for each diameter is an optional input. The computation resources required grow multiplicatively. The default number of different particle diameters run by the program is 10 . To run a study with only 1 fragment in each size category requires $1^{*} 10$ propagations, whereas to run 100 fragments per size regime requires $100^{*} 10$ propagations. By default, the number of fragments is set at 250. However, values from 50 to 1000 will work fine with limited computing power. Increasing the number for fragments will tighten the tolerance of the output fluence and lead to a narrower difference between the minimum and maximum expected flux values. It will also better assess the distribution of particles in the true anomaly graph.

The complexity of the calculations will allow the user to include or remove effects from additional planets at the expense of additional computing time. This parameter is the only input to the sys_con.m file. By default, the complexity parameter is set to 'Simple'. On this setting, Effects from the Moon, Atmospheric Drag, J2J6, Solar Radiation Pressure (SRP), Earth, and the Sun are included. For details on the other settings read the sys_con.m file. 


\section{Implementation}

The main program that accepts inputs is AsterMDO28.m. This file will prompt for all the parameters listed above. Once the inputs are accepted in the proper format, the program will call the Orbital Propagation Module.

\subsubsection{Orbital Propagation}

Many off the shelf orbital simulators are available. However, due to the need to propagate tens of thousands of fragments, a propagator was created in MATLAB. The propagator uses Cowell's method [7][34]. For higher-fidelity models, the use of an advanced orbital propagator like STK is suggested.

Main effects of the MATLAB propagator account for the gravitational attraction from the Sun, Earth, and Moon. The propagation is Heliocentric and uses the ODE45 command native to MATLAB. The position of the Earth is found by direct integration. The position of the moon is estimated using closed form solution supplied by Vallado [34]. Effects from the other planets can be included and are also computed by closed form solution from Curtis [14]. Additional effects include the oblateness of Earth do to J2 through J6 perturbations. Solar Radiation Effects assuming a spherical fragment geometry. Atmospheric drag effects are also assessed for fragments

passing below $1200 \mathrm{~km}$ altitude based on the exponential atmospheric model [34]. No drag effects are calculated for particles travelling above $1200 \mathrm{~km}$ altitude. 


\section{Implementation}

A description of the processes and procedures related to implementing the Orbital Propagator Module in MMATLAB follows.

The MATLAB files associated with Orbit propagation are: Success_Tracker11.m, calculations36.m, ShowMe2.m, P_prop_C.m, perturbed_R.m, ODE45.m, GravitySimulator23.m, Moon.m, Planetary_Ephemerides.m, planet_elements_and_sv.m, Dragmedown9.m, ZonalAcceleration2.m, and ZonalHarmonic.m.

Relevant input parameters are forwarded to Success_Tracker. Success_Tracker monitors the Orbit Propagation and Orbit Crossing Counter Modules and lets the user know that they are functioning. To propagate orbits, initial conditions for a single diameter fragment are forwarded to Calculations36. Calculations36 immediately calls $\mathrm{P}_{\text {_prop_}} \mathrm{C}$ which propagates the asteroid forward in time to its explosion date. P_prop_C could also propagate the asteroid backwards in time to an explosion date in the past, for theoretical analysis. P_prop_C calls the MATLAB native ODE45 function using the options: ('RelTol',1e-9,'AbsTol',10-12). The ODE45 function is a variable step Runge-Kutta solver which given a vector xdot, and A, (a set of equations describing how xdot relates to $\mathrm{x}$ ) it will solve for $\mathrm{x}$. In this way, the position and velocity of the asteroid can be determined at any time given an initial position, velocity, and an equation describing how acceleration and velocity changes over time. The ODE45 function acts on the function perturbed_R.

The function perturbed_R contains the equations of motion that describe how

the Earth and the asteroid particles move in space. Of primary concern is the 
accelerations experienced by the asteroid and the Earth. The position of both objects is found by direct integration. The position of other planets was assessed to determine N-body accelerations.

The positions of the Moon, Jupiter, Saturn, Mercury, Venus, Mars, Uranus, and Neptune are found by closed form solution which only requires a time input. The formulations were created by Vallado and are contained in the programs Moon, and Planetary_Ephemerides [34]. The position of Pluto is also given by closed form solution by Curtis which was implemented in the program planet_elements_and_sv. Curtis' formulation was used in this case because it had smaller errors when compared with the JPL Ephemeris estimates of Pluto's position [14].

Main accelerations can then be calculated for the asteroid and the Earth from the sun by using Equation 3.4:

$$
A=-\frac{m u}{|R|^{3}} * R
$$

Where $A$ is the acceleration, $m u$ is the gravitational constant of the sun, and $R$ is the distance from the sun to the object. N-body accelerations are calculated in a similar manner but using different $m u$ and $R$ values. This representation is valid as there is no acceleration of the XYZ (Heliocentric) coordinate system. Thus no additional terms to compensate for the coordinate system acceleration need to be included in the acceleration equations.

Accelerations on the asteroid due to atmospheric drag are added by Dragmedown9. Given an altitude, velocity relative to Earth, diameter and density, the model calculates the resulting $\delta \mathrm{V}$ due to drag. The model is exponential and is 
explained in detail by Vallado [34].

Similarly, accelerations due to non-spherical Earth, J2-J6, are included. They are derived from Vallado's work as well. The location of the asteroid is translated from XYZ (heliocentric) into IJK (Earth-Centered Inertial) and then into ECEF (Earth-Centered Earth-Fixed, also ITRF) (see Vallado). The translation to ECEF requires the current Julian date. The effects of procession and nutation are not included in this formulation. ZonalAcceleration2 performs this transformation into ECEF and then calls ZonalHarmonic with the input of the current location of the asteroid in ECEF. ZonalHarmonic calculates the resulting acceleration in the ECEF frame. ZonalAcceleration2 then translates this acceleration back into the XYZ frame by multiplying the vector with the transformation matrix in Equation 3.5:

$$
T=\left|\begin{array}{ccc}
\cos (\text { theta }) & -\sin (\text { theta }) & 0 \\
\sin (\text { theta }) & \cos (\text { theta }) & 0 \\
0 & 0 & 1
\end{array}\right|
$$

The final change in acceleration due to J2-J6 is given by Equation 3.6:

$$
\text { Del_A_ijk }=T * D e l_{-} A_{-} e c e f
$$

Where Del_A_ecef is solved for in the ZonalHarmonic.m function. Specifics on the derivation of this process are included in the appendix.

Solar Radiation Pressure (SRP) is an additional included effect on the asteroid. The calculations are based on Vallado's derivation [34]. Because asteroids are typically coming in on hyperbolic trajectories with a large velocity relative to earth, SPR 
effects are subsequently small. The calculations were further simplified by assuming no shadow effects from the Earth, the moon and other planets. An estimate of the F10.7 flux presented by Appelbaum and Flood is used [6]. These types of closed form solutions for SFU are not always the most accurate. The alternative is to use a constant flux at $1 \mathrm{AU}$ of $1366 \mathrm{~W} / \mathrm{m}^{2}$. The asteroid is assumed to be a black body with a 1.1 coefficient of reflectivity. Since the surface properties of asteroids are largely unexplored and the surface geometry is complex, diffuse, specular and absorption coefficients were not used for the SRP formulation.

All of these accelerations are summed and new positions and velocities for the asteroid and the Earth are calculated as a result within the ODE45 function. The final result, a matrix of position of Earth and the asteroid at a given time is passed up to Calculations36.m.

Orbits are propagated for 15 days by default. Longer or shorter times may be set in the AsteroMDO30.m file by manipulating the simtime parameter.

This same propagator is used to propagate asteroid fragments as well as the whole asteroid. When propagating fragments, all time steps and positions are reported to Calculations36.m. When propagating the asteroid, only the final position and velocities are reported.

\subsubsection{Critical Orbit Crossing Counter}

After propagating the fragments through their expected orbits, it needs to be determined if those fragments passed through critical orbit regimes. Critical Orbit 
Regimes are defined as common orbits for satellites and other high-value space-based structures like the International Space Station. Critical Orbits that this program checks include: $425 \mathrm{~km}$ altitude (where the ISS commonly travels), $800 \mathrm{~km}$ altitude (which contains many NRO and military satellites), $1200 \mathrm{~km}$ altitude (which is the edge of LEO), and 35,768 km altitude (where geostationary communication satellites are positioned). Medium Earth Orbit, where GPS satellites are located, was not studied in this paper because the two methods provided below are too simple to present an accurate risk assessment for a migrating constellation like GPS.

To determine if an asteroid fragment crosses a critical orbit, the IJK position information is assessed. For both LEO and GEO orbits, the fragment position is analyzed sequentially, comparing each time step to the one after it. If a time step has a radius above the critical orbit and at the next time step the radius of the fragment is below the critical radius (or vice versa), there is a possible critical orbit crossing in GEO or a guaranteed critical orbit crossing in LEO. GEO is an orbit band, so particles could cross from inside the GEO radius to outside.

If an orbit crossing is detected, the fragment position readings are translated from IJK to ECI (Earth-Centered Inertial). This coordinate system has the X axis aligned w/ the vernal equinox but the XY plane is tilted from the orbital plane to be aligned with the equator. A 1-D golden section search algorithm is then applied to determine the location of the critical orbit crossing [Figure 3.13]. For GEO, the 1-D golden section search seeks to minimize the function geox by moving from one of the points in the direction of the other point. The geox function is minimized when the altitude of the fragment matches the altitude of GEO. Other functions are 
minimized for Leo at different orbit heights.

Once the location of the critical orbit crossing is found, extra steps are required only if determining a GEO crossing. A check is conducted to determine if the particle passes within 1, 4, or 15 degrees latitude. If it does, its crossing location is saved and this particle is counted as having penetrated a critical orbit. For LEO, all crossing locations are saved regardless of latitude because the LEO orbit is a sphere not a band like GEO. The number of critical orbit crossings for each orbit is summed and the final results reported to the Statistical Analysis Module.

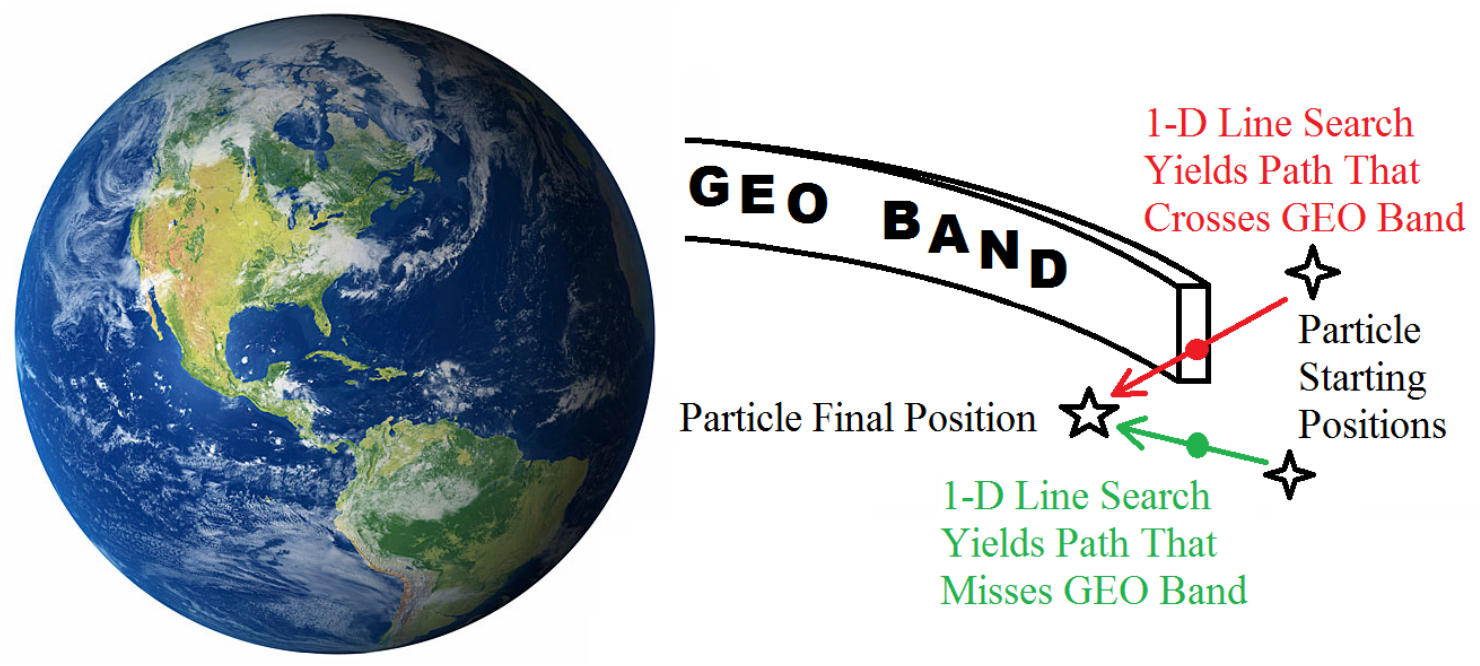

Figure 3.13: Golden Section 1-D Search Finds Where the Fragment Crosses the GEO Band 


\section{Implementation}

The files associated with critical orbit crossing analysis are: Crossing_Guard15.m, onedsrchgldsect.m, binbracket.m, geox.m, leo425x.m, leo800x.m, leo1200x.m. and Greenwich.m. The Crossing_Guard15 receives an array of fragment positions in IJK and times from Calculations36. Crossing_Guard15 identifies when a fragment crosses a critical orbit sphere. For GEO crossings only, Crossing_Guard15 then transforms the IJK fragment position to ECI (Earth-Centered Inertial) which has the X-axis aligned $\mathrm{w} /$ the vernal equinox and the X-Y plane aligned w/ the equator.

To estimate the location of the critical orbit crossing in ECI, the onedsrchgldsect program is called. This program uses a simple optimization system that searches for the lowest point of a function. This works well when there is a single global minimum and multiple local minimas do not exist. Input into this program is the location at time $\mathrm{n}$ and the location at time $\mathrm{n}+1$ of the fragment. A unit vector $S$ is created from the time $\mathrm{n}$ position in the direction of the time $\mathrm{n}+1$ position. This unit vector, $S$, is the search direction. An initial step size, $d x$, of $1 / 4$ th the distance between the two points is selected. The function to minimize is specified based on the critical orbit regime. To determine GEO crossings, the geox function is called. For LEO at 425 $\mathrm{km}$, the leo425 function is called. Similarly, leo800 and leo1200 functions are called for LEO $800 \mathrm{~km}$ and LEO $1200 \mathrm{~km}$ orbit crossings. The function is matched to the critical orbit regimes and is minimized when the radius of the fragment matches the radius of the critical orbit regime. The tolerance for the determination of the location of the critical orbit crossing is set to $1 \mathrm{~km}$ by default. Within the onedsrchgldsect, 
binbracket is also called. binbracket brackets the location of the minimum so that the 1-D golden section search can begin. The onedsrchgldsect returns the ECI location of the critical orbit crossing.

This method is a linear interpolation between the two locations of the fragment. This interpolation is most accurate when the distance between the locations is small which requires a small step size output from the Propagation Module. As the distances between the two points become larger, gravitational effects bend the line between the two locations causing a linear interpolation to become increasingly inaccurate.

One the ECI location of the critical orbit crossing is known, this point is stored in a matrix along with the linear interpolation of the time at this point. This ECI position is then converted into longitude and latitude.

The lat/longe matrix, as well as the counts of particles that crossed a critical orbit are forwarded back up to Calculations36, through Success_Tracker11, and back to AsteroMDO30. They are then forwarded to the statistical analyzer module.

\subsubsection{Statistical Analyzer}

The Statistical Analyzer Module takes the counts of critical orbit crossings (success counts) and determines a confidence interval of the number of particles expected during a disruption. The confidence interval is created separately for each fragment diameter size. In later examples, ten different fragment diameters are run. This would correspond to the creation of 10 different confidence intervals. The interval 
does not have a Gaussian distribution because there cannot be a negative number of critical orbit crossings. Therefore, a Poisson distribution is applied. Poisson distributions are applicable in situations where there is a need to determine the number of successes in a given time. It is applicable as long as each success happens independently of other successes. A single success or occurrence is defined as a fragment crossing a critical orbit once. A fragment can cross through multiple critical orbits multiple times on its trip by Earth.

\section{Implementation}

Files that relate to the Statistical Analysis Module are: StatisticsAnalyzer6.m.

Each loop of the orbit propagator creates a success count for an individual diameter. Thus if the orbital propagator is run 8 times, for particles of diameter 0.1 cm, 8 readings will be created: $[4,3,0,7,1,2,0,1]$. In this example, 500 fragments of diameter $0.1 \mathrm{~cm}$ were created, and 4 of them passed through LEO at $425 \mathrm{~km}$. Then, the propagator again and created 500 fragments of diameter $0.1 \mathrm{~cm}$, and 3 of them passed through LEO425. These values can be assessed by the MATLAB function poissfit which determines the upper, lower, and mean success value. The mean value is equal to the $\lambda$ parameter for a Poisson distribution. For the example above, a $95 \%$ Confidence Interval (CI) is found to be $(3.35,1.33)$ with a mean of 2.25. This signifies that it is expected that 3.35 to 1.33 out of every 500 particles to pass through a LEO425. This indicates that $(0.71 \%, 0.27 \%)$ of particles of this size will pass through the critical orbit. Graphs of the different critical orbit regimes 
and the CI's associated with them can be supplied at every loop of the propagation by removing the comments in front of the line that calls StatisticsAnalyzer6.m (see MATLAB Code).

If there is a large percentage of zero successes, StatisticsAnalyzer6 suggests to the user that increasing the number of fragments per propagation may be required. This is to increase the number of critical orbit crossings to a detectable level. Increasing the number of particles per propagation will not alter the underlying physics that determine if a particle passes through a critical orbit.

The resulting CI's for each diameter (see Figure 3.14) are forwarded back to AsteroMDO30 which then passes them on to the MMOD Fluence Analyzer. 


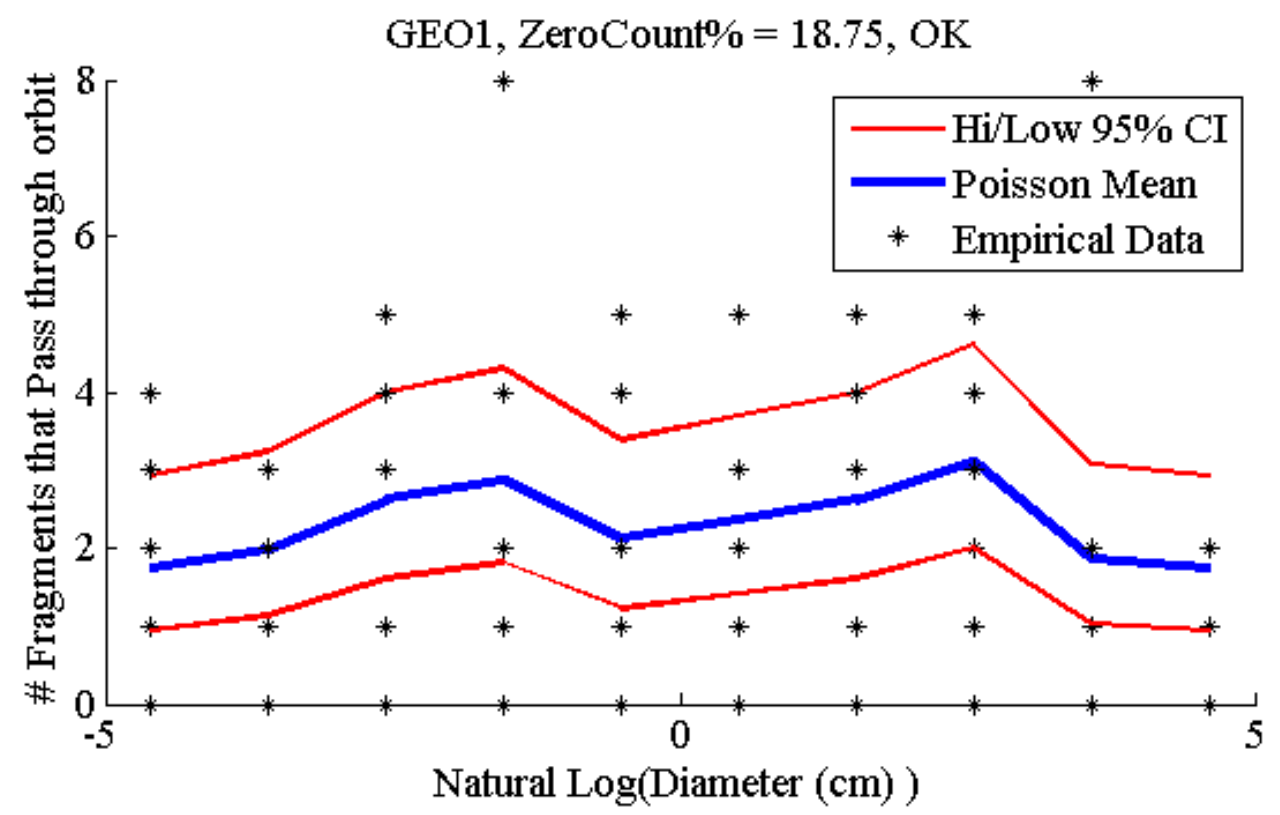

Figure 3.14: Example of Individual Poisson Distribution Fits for Each Fragment Size Bin

\subsubsection{MMOD Fluence Analyzer}

The MMOD Fluence Analyzer Module assesses the transient fluence due to asteroid disruption. Fluence is the number of particles that pass through a given area of space $\left(\# / m^{2}\right)$. Flux is the number of particles that pass through an area over a given amount of time $\left(\# /\left(m^{2} *\right.\right.$ time $\left.)\right)$. Additionally, the module assesses the typical daily MMOD Fluence levels. It then compares the transient fluence against the typical fluence. This analysis is then summarized and passed through to the Output Parameters Module for visual display. 


\section{Implementation}

The files associated with the MMOD Fluence Analyzer are: Flux_Analyzer4.m, PowerCurve3.m, MM_Tribble.m, and OD_Kessler.m.

Flux_Analyzer3 accepts the CI created by the Statistical Analyzer Module. This data contains the $95 \%$ confidence interval for each fragment diameter bin. With the logspace bin size setup in the program, it is typical that diameter bins would be setup in the following sizes: $[100.00,35.94,12.92,4.64,1.67,0.60,0.22,0.08,0.03$, 0.01]. All values are given in centimeters. These sizes are initially created in the AsteroMDO30 program using MATLAB's logspace command.

Bins of particles are crated based on the above particle sizes in the MMOD Fluence Analyzer Module. Size bins are allocated as follows: The number of particles in the $100 \mathrm{~cm}$ diameter bin is the cumulative number of particles $100 \mathrm{~cm}$ diameter or larger. The number of particles in the $35.94 \mathrm{~cm}$ bin is the cumulative number of particles of sizes $35.94 \mathrm{~cm}$ to $100 \mathrm{~cm}$. This trend continues down to the smallest bin, $0.01 \mathrm{~cm}$, which contains the total number of particles of size $0.01 \mathrm{~cm}$ to $0.03 \mathrm{~cm}$. So for the $100 \mathrm{~cm}$ bin, this assumes that particles larger than $100 \mathrm{~cm}$ in diameter experience the same orbital effects as spherical particles $100 \mathrm{~cm}$ in diameter. This simplification creates small inaccuracies in drag and SRP calculations. However, the total number of particles from a disruption expected to be in this bin is typically on the order $1 \%$ or less of the total number of particles created. Thus, even if particles of $2 \mathrm{~m}$ in diameter experience radically different trends than those of $100 \mathrm{~cm}$ in diameter, there are too few of them to cause any significant changes in the overall 
CI's produced by this program. This is because there are orders of magnitude more smaller particles in the $0.01 \mathrm{~cm}$ to $1 \mathrm{~cm}$ size bins that contribute disproportionately to the overall CI fluence estimate given in the Output Parameters.

The total number of particles in each bin is calculated using PowerCurve3. This produces a count of the cumulative number of particles that would be created above a specified diameter based on initial asteroid mass, density, and largest remaining chunk of the asteroid. The formulation is based on a power law presented by Sanchez, Vasile and Radice [27]. The cumulative distribution is changed to non-cumulative which yields the total number of particles in each size bin.

The CI percentages for each bin are multiplied by the total number of particles in that bin. This value is then dividing by the surface area of the respective critical orbit. The result is multiplied by the time duration of the transient fluence (typically assumed at 1 day). This yields an upper and lower 95\% CI for the fluence in each particle size bin in each critical orbit. The cumulative fluence for $0.1 \mathrm{~cm}$ diameter particles (in GEO) and $1 \mathrm{~cm}$ diameter particles (in LEO) is then calculated.

The Micrometeoroid fluence is calculated using the Grun model. This model is contained in the MM_Tribble program and provides a closed form solution to MM fluence in LEO and GEO orbits. This model has an upper and lower interval. The upper half of the interval is created by doubling the previous result. This is consistent with USAF guidelines for conservative estimates of MM fluence [25]

The orbital debris fluence is calculated using the Kessler model. This model is contained in the OD_Kessler program. It provides a closed form solution to the 
estimated orbital debris in LEO orbits. OD in GEO orbits is not included in this model. This program accounts for debris-debris interaction and magnification of already existing debris counts. It does not take into account any future debris miti-

gation efforts. A smoothed 13 month solar flux formulation by Suggs is used [28] to determine Solar Flux Units (SFU). By default, this program determines the fluence experienced on a tumbling satellite surface orbiting at 28.5 degrees inclination (an equatorial orbit). Typically ram-facing surface have a higher flux that wake-facing or tumbling surfaces.

Several summary matrices with the final fluence values for each size bin are forwarded to the Output Parameters Module where they will be graphed.

\subsubsection{Output Parameters}

The Output Parameters Module summarizes the information provided from other sources and displays them for easy ingestion by the user. This information is displayed for each critical orbit regime. The information displayed includes: Transient fluence vs. daily fluence text, Transient fluence vs. daily fluence graph, Heatmap with latitude longitude penetration locations.

\section{Implementation}

The files associated with the Output Parameters Module are: Output_Parameters6.m, and heatmap2.m. 
Initially, Output_Parameters6 pulls information out of the matrices and cells prepared my the MMOD Fluence Analyzer Module. This data is stitched together with text to make the transient fluence vs. daily fluence text output that is sent directly to the MATLAB command screen (for examples see Figure 5.1 and Figure 8.1). Graphs of this same data are output to the screen using the MATLAB plot command.

A graph of the percentage of particles that pierce through different longitude bins is assessed by pulling data from the Intercept_ld matrix. Longitude bins are separated into five degree bins. A histogram is then created based on the critical orbit piercings in each bin. This information is displayed along with text describing the duration of the event. This is calculated by taking the difference between the first particle that pierces a critical orbit and the last particle that pierces a critical orbit.

A map of all critical orbit piercings is then created by calling the heatmap file. This plot is referred to as the heatmap in later sections. For GEO orbit bands, this file breaks up the latitude and longitude of the GEO orbit into $72 \times 5$ boxes. Tallies are taken of the number of times each box is pierced by a fragment. The sums are then matched to a meshgrid of points for 0:360 degrees in increments of five degrees for longitude, and -12.5 to +12.5 degrees for longitude. Each point on the grid is given a value corresponding to the bin closest to it. The final output is displayed using the surface command built into MATLAB.

Similarly, a heatmap for all critical LEO orbits is created. However, since the 
LEO orbit creates a sphere, not a band, the number of bins decreases as the absolute value of latitude increases. Thus at the poles there are only four bins, whereas at the equator, there are 72 . This allows the map to flatten so that a spherical surface can be projected on a flat plane. The resulting bin counts are found in similar style to the GEO process highlighted in the previous paragraph. Final display is sent to the user via the surface command in MATLAB.

\subsection{Assumptions}

The following is a list of assumptions that were made for this analysis:

- Transient fluence levels due to disruption for every critical orbit regime will vary based on deflection scenario

- The orbital parameters and physical properties of the scenarios presented in Fic2029-400, Fic2029-500, and Fic2029-600 are reasonable approximations of asteroids on a collision course with Earth. However no asteroids with these parameters actually exist.

- By 2029, nuclear surface and subsurface disruptions have been tested on nonEarth-crossing asteroids

- Russia, the EU, China, and the US will work together to deflect a potentially hazardous asteroid 
- The total number and size of particles coming from an asteroid disruption can be modeled with a one segment power law. (To refine this, details on internal asteroid structure and composition are required.)

- Any disruption effort will create a Largest Remaining Chunk (LRC) of $5 \mathrm{~m}$ to $20 \mathrm{~m}$ in diameter

- The asteroid mass is known

- Asteroids and asteroid particles are black bodies with a 1.1 coefficient of reflectivity

- Asteroids and asteroid particles are assumed to be spherical

- Asteroids and asteroid particles have uniform densities

- The orbital propagator is a perfect model and contains no errors. Thus the fluence CI does not account for variations due to orbital uncertainty.

- Closed form solutions for the moon and other planets are accurate representations of their true locations

- The JPL ephemeris database has better accuracy than the MATLAB propagator used for this study

- Particles that pass below $100 \mathrm{~km}$ altitude burn up in the atmosphere or hit the surface of Earth and do not travel back into space

- The disruption date will be accurately determined to the second 
- There is no uncertainty in the asteroid's position and velocity

- Gravitational effects between asteroid particles are insignificant because the asteroid is catastrophically disrupted

- The velocity of particles coming from the explosion does not depend on the mass of the particle

- The location of the nuclear explosion on the asteroid has no effect on velocity unit vector of particles issuing from that explosion

- The unit vector of the change in velocity of asteroid particles is randomized about 3 axis (eight quadrants)

- The center of the Sun is at the solar system barycenter and does not move

- No particles pass below the radius of the moon or other planets. (The program does not currently destroy these particles.)

- The effects of the Earth's nutation and procession are negligible

- The time step used in this analysis is small enough that $100 \%$ of critical orbit crossings are identified

- Over small enough time steps, particle motion is assumed to be linear

- A Poisson distribution is a reasonable representation of the fluence in each size bin 
- Particles in each size bin behave like the typical sized particle for that bin. i.e. All particles in the size bin of 0.1 to $0.2 \mathrm{~cm}$ diameter behave the same as 0.1 $\mathrm{cm}$ particles. Only $0.1 \mathrm{~cm}$ particles (typical size for this bin) are propagated in the orbital propagator.

- Current fluence levels are accurately approximated with ORDEM2000 and Grun models

- Typical orbital debris fluence in LEO is based on a tumbling spacecraft in a 28.5 degree inclination 


\section{Chapter 4}

\section{Program V and V}

\subsection{Verification}

Verification is the process of ensuring that a product is constructed correctly for that type of product.

\subsubsection{Propagator Module}

Three verifications of the orbit propagator module were conducted by measuring the residual of the propagator against a reference, the JPL Ephemeris database. The elements that were checked include the propagation of Earth, the Moon, and the asteroid Apophis.

The residual of the Moon's position from the closed form solution shortened 
Earnest Brown method presented by Vallado is analyzed to assess its accuracy [34]. The resulting comparison against JPLs data, which is assumed to be of much higher accuracy, is contained in Figure 4.1. Notice that there is a cyclical behavior of the error which oscillates about $2.2+\mathrm{E} 4 \mathrm{~km}$. This indicates that this method can be used to estimate the moon's position. However, for the accuracy of future studies, more accurate estimators should be used. The shortened Earnest Brown method will suffice to validate the framework's function as the error is bounded and has predictable characteristics.

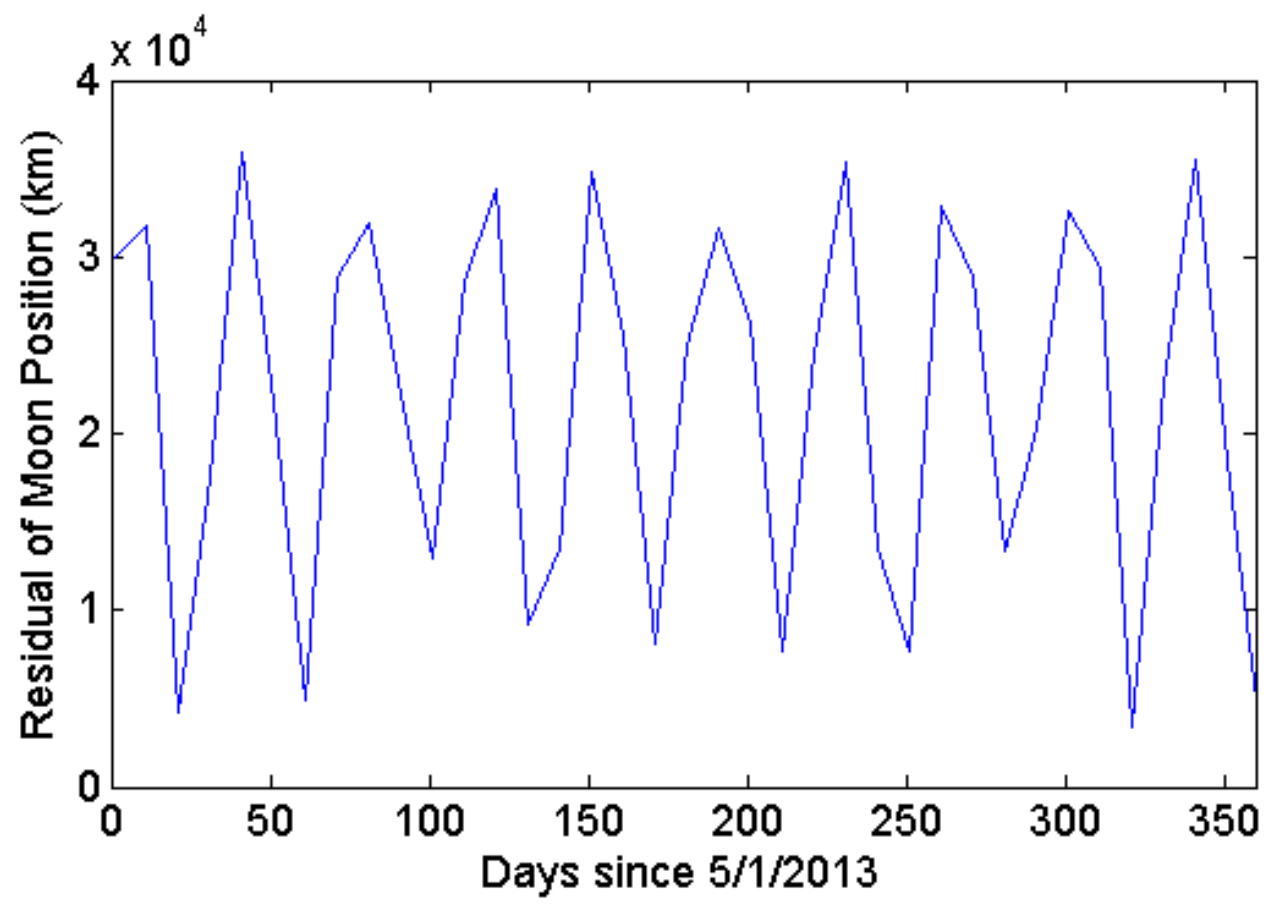

Figure 4.1: The Residual of the Moon over a Year's Time

The residual of the Earth's position when comparing Cowell's method solution vs. 
the JPL ephemeris is presented in Figure $4.2 \&$ 4.3. It is noted that the residual for the years of $2029 / 2030$ is bounded at roughly a $2 \%$ error relative to one AU. However, since the propagation happens over a fifteen day stretch starting at $4 / 1 / 2029$, the residual is actually much smaller. The residual for this short time period is around $0.04 \%$.

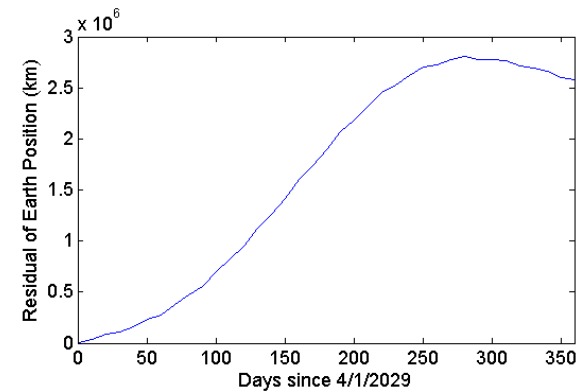

Figure 4.2: The Residual of the Earth over a Year's Time

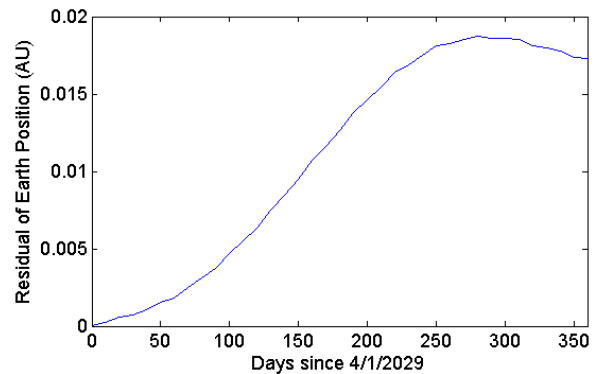

Figure 4.3: The Residual of the Earth over a Year's Time

To assess the overall performance of this propagator when it comes to complex asteroid bodies a test case is introduced. The asteroid 99942 Apophis, also known as 2004 MN4 has been closely monitored and orbital elements have been narrowed down significantly compared to other Potentially Hazardous Objects (PHO). A comparison was made against the estimate of the location of Apophis against the JPL Ephemeris Database to determine a residual and thus the accuracy of this propagator [31]. The data contained in Figure 4.4 show that over a year's time, the residual climbs in an uneven pattern. Examining this over a longer timescale of two years (Figure 4.5) and increasing the complexity of the propagator to medium, it is noted that it rises and falls. This suggests that there are significant effects on this asteroid's trajectory that 
are not contained in this propagator. However, for time scales on the order of fifteen days, the residual for simple \& complex propagation is on the order of $59.3 \mathrm{~km}$ to $202.6 \mathrm{~km}$. For short-term fluence estimates, this error is acceptable as it represents less than 1/32nd of Earth's diameter.

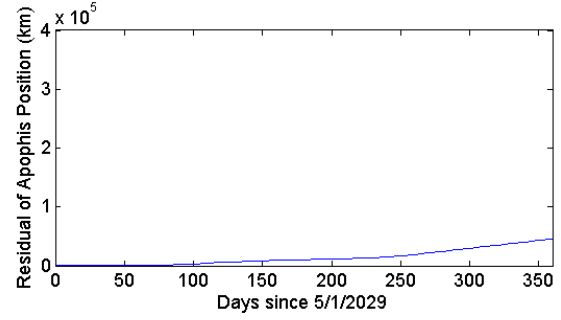

Figure 4.4: The Residual of the Apophis over a Year's Time

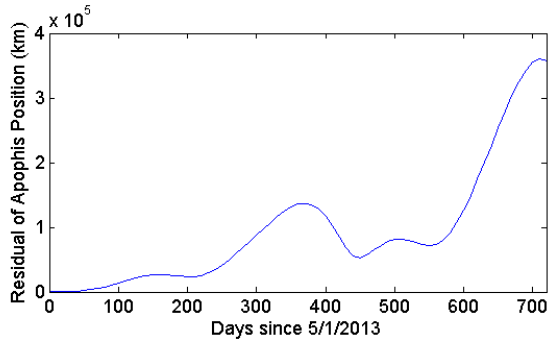

Figure 4.5: Residual of the Apophis over Two Years

However, the above test were conducted for a dates starting at 5/1/2013 and onwards, when Apophis and Earth had 0.324 AU of separation. The encounter of Earth and Apophis in April of 2029 will be much closer so this case will also be examined. Figure 4.6 contains the results from propagating Aopohis through its close encounter with Earth in 4/15/2029. What is noticed is that the error increases sharply just prior and post the encounter. This may be a combination of effects from the position of the Moon as well as the fact that this propagator model does not include a 70x70 gravity matrix. The J2-J6 effects were the only ones included for speed in the MATLAB environment. The initial error is very small because the initial location of the asteroid in the propagator is set to the location in the JPL database. 


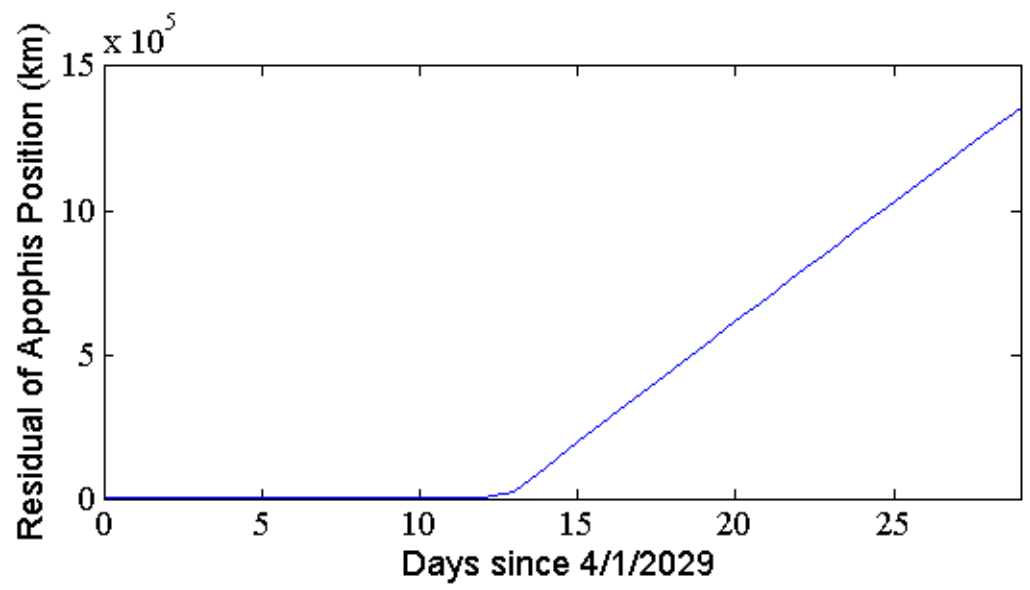

Figure 4.6: Residual of the Apophis over 30 days

While there may be subtle issues with the complexity of the propagator, this will not prevent the validation of the framework as whole and showing that the methods of analysis conducted here are capable of defining the change in the space environment.

\subsubsection{Critical Orbit Crossing}

Verification of the critical orbit crosser was done by creating test fragments and propagating those fragments through their encounter with Earth. Using the MATLAB plot3 function, the location of the particles was plotted in 3D along with a spherical Earth. If a critical orbit crossing in any of the LEO regimes happened, an additional sphere was plotted which represented that critical orbit. The fragment trajectory was then visually examined to verify that it crossed through the critical orbit. This check, performed on over fifty LEO critical orbit crossings yielded by 
inspection that the critical orbit crossing software was successfully detecting critical orbit crossings.

A similar inspection process was implemented to examine particles that no particles were propagated below $100 \mathrm{~km}$ altitude. At this point, we assume they have burned up in the atmosphere. The trajectory of fragments was plotted using the plot3 command in MATLAB. A sphere at $100 \mathrm{~km}$ altitude about the Earth was plotted as well. Any fragment that passed within this sphere was observed to cease propagation and cause the burn up counter to increase by one. The burn up itself was achieved using MATLAB events. The effect on the critical orbit crossing counter was that critical orbit crossings were detected as the fragment plunged towards 100 $\mathrm{km}$ altitude. After the fragment burned up, no additional orbit crossings were detected. Thus, the critical orbit crossing counter module was found by inspection to be functioning correctly.

\subsubsection{Velocity Distributions}

To verify that the velocity distribution functions being constructed where representative of the original velocity distributions, the original distributions is plotted side by side with a representative sample of points created from the distribution function. In the graphs below, the original Weaver2.m distribution is compared against the approximation of the distribution contained in the Weaver2.m. Figure 4.7 shows the original distribution supplied from Weaver. Figure 4.8 shows the file after creating 1,000 points using this file. The two figures appear to have similar distributions. 
This shows that indeed this method for creating velocity distributions from original velocity curves works correctly.

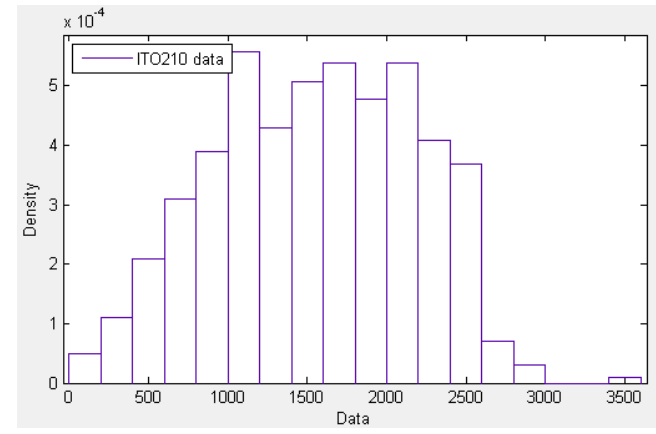

Figure 4.7: Original Weaver2 Distribution

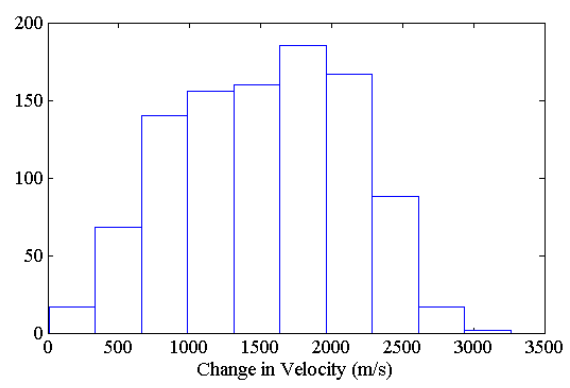

Figure 4.8: Simulated Weaver2 Distribution

\subsection{Validation}

Validation is the process of ensuring that a product meets the customers need.

To ensure that the program produces repeatable results, the Fic2029 example was run in its entirety three times. The expectation is that the output of the second and third runs would complement the analysis from the first run and not produce wildly different results. The input parameters for the first Fic2029 run are located in Table 5.1. The input parameters for the second and third study are located in Table 8.2. The critical parameter that can be used to quickly compare these three studies is the CI created for fluence at each critical orbit. The comparisons are contained in Table 4.1. GEO1 represents the GEO +/- 1 degree band. GEO4 represents the GEO +/- 4 degrees band, and GEO15 represents the GEO +/- 15 degrees band. 


\section{Table 4.1: Results Comparison, Three Fic2029-500 Disruption Studies}

$\begin{array}{ccccc}\text { Critical Orbit } & \text { Study 1 } & \text { Study 2 } & \text { Study 3 } & \text { Units } \\ \text { LEO425 } & (1.3,3.8) & (0.4,1.2) & (0.5,1.4) & \text { days } \\ \text { LEO800 } & (0.7,2.0) & (0.2,0.7) & (0.3,0.8) & \text { days } \\ \text { LEO1200 } & (0.6,1.6) & (0.3,0.7) & (0.3,0.8) & \text { days } \\ \text { GEO1 } & (1.5,7.7) & (1.7,6.9) & (0.9,4.7) & \text { years } \\ \text { GEO4 } & (0.3,1.2) & (0.4,1.1) & (0.3,0.9) & \text { years } \\ \text { GEO15 } & (29.8,80.3) & (27.1,69.1) & (24.7,64.2) & \text { days }\end{array}$

The results for study two and three are included in the MATLAB zip file which also contains the program code. Table 4.1 shows that for all the GEO fluence values, the CIs overlap. This is exactly what was expected for high-fluence effects which are easy to detect with just 500 particles per run. When the transient fluence is on the order of years, this can have significant effects on spacecraft survivability. The fact that the CIs for the LEO orbit regimes do not all overlap is not concerning. The differences in statistics between model runs are consistent with the sample variability that might be expected under the model scenarios. With expected fluence in the regime of 30 days or less, the change in the space environment is so small as to be inconsequential when calculating spacecraft survivability. Additionally, it is not surprising that all the LEO regimes do not overlap simultaneously as the same particles that are passing through LEO $1200 \mathrm{~km}$ are those that could pass through LEO 425 km. Thus lower particles passing through LEO1200 signify that the LEO425 regime would also typically experience decreased fluence. 


\section{Chapter 5}

\section{Example Disruptions}

In this chapter a few examples of asteroid disruptions will be presented. The framework outlined so far will be used to study the MMOD effects of deflecting the asteroid. Input and output parameters will be shown as well as intermediate steps. The first example of the asteroid 'Fic2029' is roughly based on the close approach of Apophis at this same time but initial velocity conditions have been modified to take the asteroid closer towards earth.

The fluence resulting from asteroid disruptions is highly scenario dependent. The results contained herein do not represent the expected fluence levels for every deflection scenario. They represent only the expected results for this specific fictitious scenario. There is no known asteroid on a collision course with Earth. And any the results of any future deflection would be expected to be different than the example results provided below. The fictive examples are provided only as a means of 
demonstrating the capabilities of the novel framework presented in this paper.

\section{$5.1 \quad$ Fic2029-500}

In this fictive example, an Itokawa-type asteroid 'Fic2029' is detected on April 1, 2029. The asteroid is expected to make a close pass to Earth on April 14, 2029. With only a few observations available of the asteroid, the expected closest approach distance is around 6,000 $\mathrm{km}$ altitude. This is less than three Earth Radii and with additional uncertainty in the orbit, it appears that there is a 1 in 1000 chance of an Earth-impact. The asteroid itself is estimated to be a rubble-pile some 400 meters in diameter with an assumed average density of $1.9 \mathrm{~g} / \mathrm{cm} \hat{3}$ and a total mass of $3.51^{*} 10 \hat{1} 0$ $\mathrm{kg}$.

It is assumed that by 2029, nuclear surface and subsurface disruptions have been tested on non-Earth-crossing asteroids and thus are available for short-mitigation times. Additionally, only $85 \%$ of the interception tests have been successful, with $15 \%$ of test missing their mark. The UN has passed a resolution that supports disruption with nuclear weapons against asteroids that pose a 1 in 5,000 risk of hitting the Earth and that cannot be deflected by other means. Even with only a 1 in 1000 chance of the Asteroid impacting Earth, this risk is deemed too high and so an international disruption effort is readied which involves two interceptors. This will allow only a $2.25 \%$ chance that both interceptors miss.

Both interceptors are equipped with a $500 \mathrm{kt}$ warhead which is set to detonate 
on surface impact. One interceptor is launched by Russia and is expected to hit the asteroid on April 9th. The other is launched by the US and is expected to hit the asteroid on April 11th. The effects of either the Russian or the US interceptor hitting the asteroid and the resulting effects on orbiting satellites is summarized in the Fic2029 example below.

\subsubsection{Input Parameters}

Assuming that the first disruption attempt on April 9th is successful, the following parameters in Table 5.1 are inputs to the program: 
Table 5.1: Input Parameters, April 9th Disruption

\begin{tabular}{|c|c|c|}
\hline Explosion Date & {$[2029,4,9,0,0,0]$} & {$[$ year,mm,dd,hh,mm,ss] } \\
Observation Date & {$[2029,4,1,0,0,0]$} & [year,mm,dd,hh,mm,ss] \\
\hline Position Earth (X) & $-9.803428546825277 \mathrm{E}-01$ & AU, Heliocentric \\
Position Earth (Y) & $-1.930901065107917 \mathrm{E}-01$ & AU, Heliocentric \\
Position Earth (Z) & $2.078772183457271 \mathrm{E}-05$ & AU, Heliocentric \\
\hline Velocity Earth (X) & $3.040684386769381 \mathrm{E}-03$ & AU/day, Heliocentric \\
Velocity Earth (Y) & $-1.693819813500764 \mathrm{E}-02$ & AU/day, Heliocentric \\
Velocity Earth (Z) & $8.062826223939135 \mathrm{E}-07$ & AU/day, Heliocentric \\
\hline Position Asteroid (X) & $-1.012505408225337 \mathrm{E}+00$ & AU, Heliocentric \\
Position Asteroid (Y) & $-2.212009168899217 \mathrm{E}-01$ & AU, Heliocentric \\
Position Asteroid (Z) & $-1.209751944049115 \mathrm{E}-02$ & AU, Heliocentric \\
\hline Velocity Asteroid (X) & $5.596108154750269 \mathrm{E}-03$ & AU/day, Heliocentric \\
Velocity Asteroid (Y) & $-1.481348469905512 \mathrm{E}-02$ & AU/day, Heliocentric \\
Velocity Asteroid (Z) & $9.217139513703808 \mathrm{E}-04$ & AU/day, Heliocentric \\
\hline Asteroid Mass & $3.51 \mathrm{E}+10$ & $\mathrm{~kg}$ \\
Asteroid Volume & $3.28736 \mathrm{E}+7$ & $\mathrm{~m}^{3}$ \\
Asteroid Density & 1.05 & $\mathrm{~g} / \mathrm{cm}^{3}$ \\
\hline Largest Remaining Chunk & 20 & meters \\
Explosion Profile & Weaver1.m & string \\
Fragments per Bin & 500 & number \\
\hline Propagation Loops & 8 & - \\
\# Fragment Bins & 10 & - \\
Total \# Fragments & 40,000 & - \\
\hline
\end{tabular}

Assuming that the second disruption attempt on April 11th is successful, almost the same parameters are input in the program. The only change is that the explosion date is altered from 4/9/2029 to $4 / 11 / 2029$ (see Table 8.1). 


\subsubsection{Results - April 9 Disruption}

The first results that MATLAB gives us as the program processes is the counts of how many particles passed through critical orbits. This is displayed through the ShowMe2 program and it will keep us updated on the programs process through all the calculations (see Table 5.1.2). In the upper left corner, the current propagator run number is displayed. The time remaining estimate is coded into the program and is based on the time to run a 15 day propagation on an Intel Core i3 laptop (1.2 seconds). It is possible to edit this value in the ShowMe2.m file.

$\begin{array}{rlrrrlr}\text { Frag\# } & \text { LEO } & \text { GEO1 } & \text { GEO4 } & \text { GEO15 } & \text { Fdiameter } & \text { Fdensity } \\ 500 & 4 & 2 & 14 & 30 & 100 & 1.04744 \\ 500 & 3 & 2 & 6 & 23 & 35.9381 & \\ 500 & 2 & 1 & 5 & 21 & 12.9155 \\ 500 & 8 & 2 & 13 & 35 & 4.64159 \\ 500 & 4 & 4 & 8 & 26 & 1.6681 \\ 500 & 2 & 1 & 5 & 16 & 0.599484 \\ 500 & 1 & 1 & 5 & 17 & 0.215443 \\ 500 & 5 & 3 & 7 & 26 & 0.0774264 \\ 500 & 8 & 2 & 6 & 21 & 0.0278256 \\ 500 & 3 & 1 & 2 & 16 & 0.01\end{array}$

Table 5.2: ShowMe2.m Progress Report Displayed in MATLAB

While interpreting this figure, it should be noted that the program has estimated the density of particles based on a uniform mass distribution and a spherical fragment 
shape. 500 particles were run for each size from $100 \mathrm{~m}$ diameter through $0.01 \mathrm{~m}$ diameter. For particles that were $0.01 \mathrm{~m}$ diameter, of the 500 particles that were propagated, there were 3 crossings through the LEO1200 orbit regime or lower, one crossing through the GEO1 regime, two crossings through the GEO4 degrees orbit regime, and 16 crossings through GEO15 degrees.

Comparing the summarized CI from both the April 9th and April 11th disruption (Table 5.3) it can be seen that there is an increase in the fluence due to disruption on the 11th. The April 11th results are included in the Appendix (see Section 8.1.2). Source data for Table 5.3 is taken from Figures $5.7 \& 8.43$.

Table 5.3: Results Comparison, April 9th vs. April 11th, 2029, Disruption Studies

$\begin{array}{cccc}\text { Critical Orbit } & \text { CI, April 9th } & \text { CI, April 11th } & \text { Units } \\ \text { LEO425 } & (1.3,3.8) & (5.9,8.0) & \text { days } \\ \text { LEO800 } & (0.7,2.0) & (3.2,4.2) & \text { days } \\ \text { LEO1200 } & (0.6,1.6) & (3.0,3.9) & \text { days } \\ \text { GEO1 } & (1.5,7.7) & (5.0,13.3) & \text { years } \\ \text { GEO4 } & (0.3,1.2) & (1.3,3.0) & \text { years } \\ \text { GEO15 } & (29.8,80.3) & (103.8,225.2) & \text { days }\end{array}$

The GEO1 critical orbit regime is where the majority of GEO satellites reside. An April 9th disruption would cause between 1.5 and 7.7 years worth of fluence to travel the regime per day for 1.9 days. Thus the satellite would be significantly weathered. Delaying the disruption until April 11th appears to shift the CI upwards significantly to 5.0 to 13.3 years worth of fluence per day for 1.3 days. However, just because the mean of the April 11th study is larger than the April 9th study does not 
indicate that the results are statistically different. Since the CI's overlap, additional studies would be needed to narrow down the CI and show conclusively if an increase in fluence due to later disruption is expected.

\section{Side Effects - LEO $425 \mathrm{~km}$}

Presented here are the results of the April 9th, 2029 successful disruption and its effects on the LEO $425 \mathrm{~km}$ critical Orbit. Similar graphs for LEO $800 \mathrm{~km}$ and LEO $1200 \mathrm{~km}$ are presented in the Appendix (see Section 8.1).

The first results from each critical orbit, Figures $5.1 \& 5.7$, are given in the MATLAB command window and contain the upper and lower Confidence Interval of expected fluence. This CI is a critical parameter that describes how the space environment is expected to change based on the disruption.

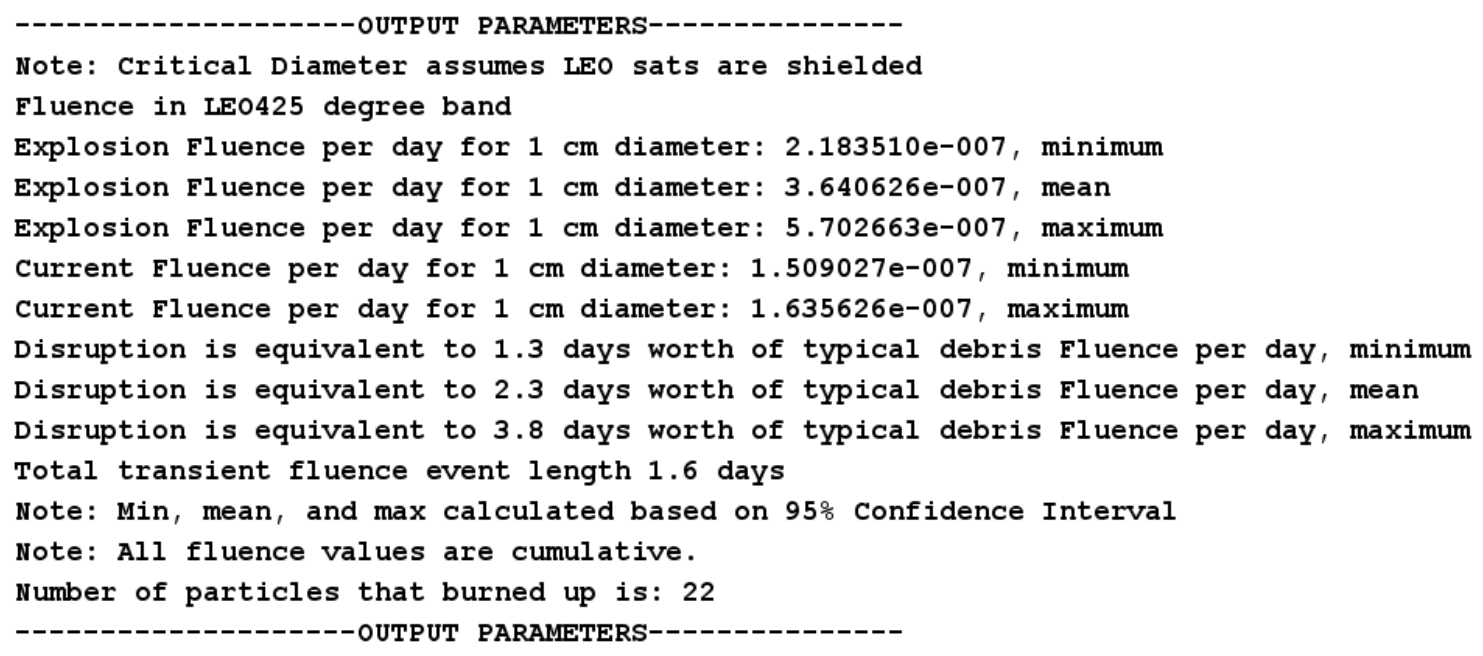

Figure 5.1: MATLAB Command Line Output 
The graphs titled Mean MMOD Comparison, Figures $5.2 \& 5.8$, show the current estimate of the MMOD Fluence in the critical orbit regime compared with the transient MMOD created by the disruption. During both disruption dates, the LEO typical MMOD and disruption MMOD match closely at the critical diameter for this orbit $(1 \mathrm{~cm})$. From the GEO graphs for both disruption dates, the transient fluence is orders of magnitude larger than the typical for the critical diameter at this orbit $(0.1 \mathrm{~cm})$. Both these observations are expected due to the characterization from the MATLAB text output.

The graphs titled MMOD CI Comparison, Figures $5.3 \& 5.9$, show that the CI for each of the disruption days and orbit regimes. The blue lines about the red transient MMOD fluence line represent the CI of expected fluence values. It is noticeable that these graphs look quite similar to the Mean MMOD Comparison graphs and that the CIs represented in these graphs are quite narrow. Thus, it is easy to determine if there will be a large or small difference in transient fluence because the CIs for the transient and typical fluence rarely overlap.

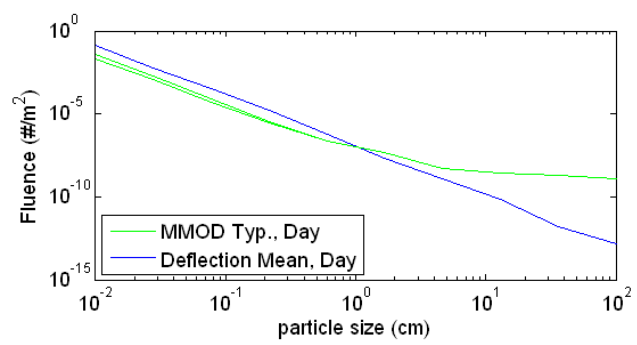

Figure 5.2: Mean MMOD Comparison

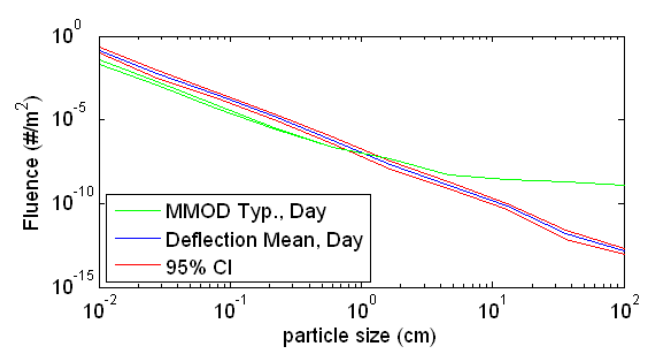

Figure 5.3: MMOD CI Comparison

The Original Asteroid Orbit Check Plot, Figure $5.4 \&$ 5.10, is a sanity check plot. 
The red line representing the original path of the asteroid should always cross within the green circle which represents Earth. If this is not the case, then it is possible that the asteroid was not initially on a collision course with Earth and thus a disruption was not required. This graph is meant as a cursory check and does not guarantee that the asteroid would have hit Earth. To perform this analysis, a 3D plot would need to be created of the orbital path which is difficult to shown in this paper format.

The Longitude Bin Penetration Percentage, Figure $5.5 \& 5.11$, shows the percentage of particles that penetrate each longitude bin. The percentage is based on the total number of particles that cross the specified critical orbit regime. These graphs allows us to immediately see trends of high-penetration areas. A large spike in fragment penetrations in a single longitude bin would indicate that spacecraft in this longitude bin would be disproportionately affected by the disruption. However, the graphs show that the distribution is generally flat and thus there is not a large difference between the longitude bins. There is a slight dip in the penetrations at 320 degrees East longitude which may indicate that spacecraft in these bins will receive less fluence than the rest of the regime. In other disruption examples we see other trends such as a peak in Figure 5.4 and a dip in Figure 8.29.

The Heatmap graphs, Figure $5.6 \& 5.12$, give further details the location of critical orbit penetrations. The color of the box represents the percentage of particles that travelled through that critical orbit box. The percentage is calculated against the total number of particles that have been propagated from the asteroid explosion. No confidence interval is calculated for this percentage. 


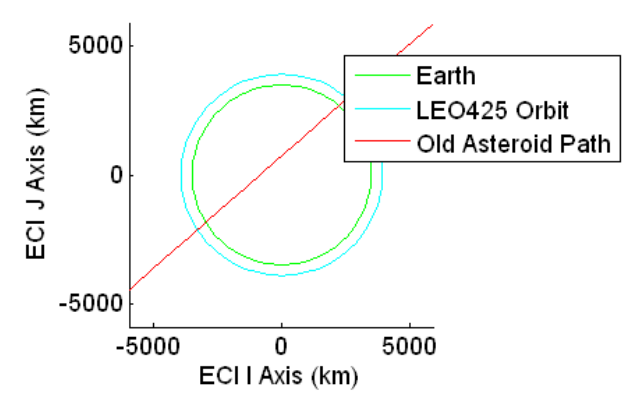

Figure 5.4: Original Asteroid Orbital Path Check

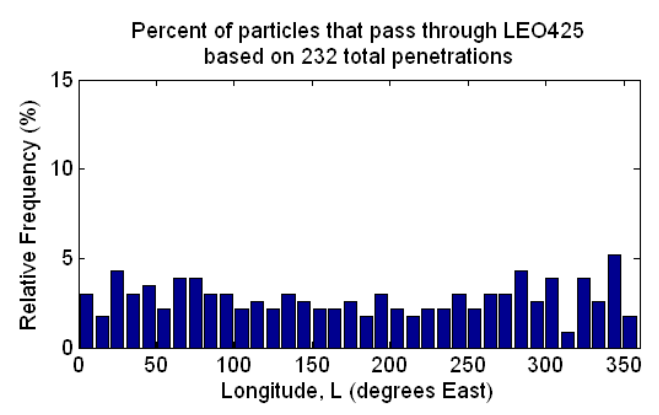

Figure 5.5: Longitude Bin Penetration Percentage

For the LEO graphs, the scattered nature of each penetration indicates that no single area will receive a higher fluence due to disruption. For the GEO graphs, there appears to be a disproportionate distribution of particles penetrating the orbit regimes of 150 to 200 degrees East longitude. This difference is exacerbated by the April 11th disruption and extends through to 75 to 225 degrees East longitude (see Figure 8.48). A larger percentage of particles is expected to travel through these GEO bins. This is an important fact for GEO spacecraft operators with spacecraft in these bins as it may be prudent for them to relocate their equipment for the duration of the transient fluence.

While both of these example scenarios have high fluence levels in the GEO1 150 to 200 degree East longitude bins, this is an effect of initial orbital trajectory of the asteroid relative to Earth. Different asteroid will have different trajectories and will therefore cause increased fluence in varying longitude bins. That is to say, the three spacecraft listed in Figure 5.12 will not always feel the brunt of the effect of an asteroid disruption. 


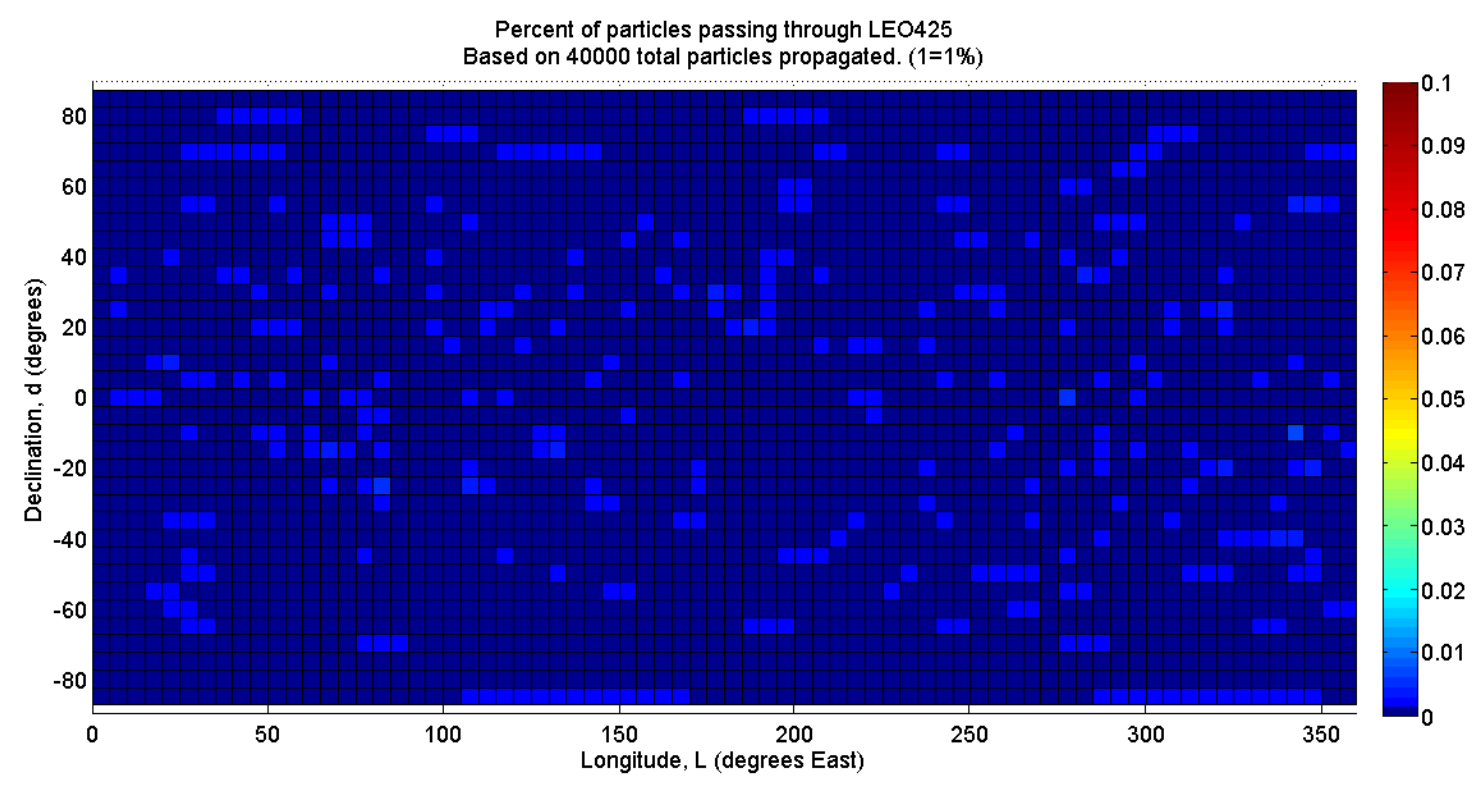

Figure 5.6: Heatmap of Penetrations of Critical Orbit

There is a critical difference in the Heatmap from LEO425 and GEO1 (see Figures $5.6 \& 5.12$ ). The LEO heatmap has penetrations spread about the sphere with no one area of high-concentration. The GEO Heatmap shows a larger percentage of penetrations in the region of 150 to 200 degrees East longitude. Additionally, in other studies we run for different explosion strengths and different days, this trend of high-fluence persists. GEO satellites stay in a single longitude bin. Between 150 and 200 degrees West longitude there are 23 operational GEO Satellites. If this disruption were to occur, these satellites would be disproportionately affected. There are satellites in these GEO longitude bins that provide critical services to many countries. Cosmos 2479 is one of four satellites that comprise the Russian ICBM 
early-warning system. USA 195 supplies satellite communicates for the Pacific fleets of the US and Australia. TDRS-10 is a NASA satellite that is a communication hub for other satellites. Figure 5.12 shows the position of these satellites relative to the high-fluence areas. A loss of one or several of the satellites in these GEO bins could be crippling to the communications network.

\section{Side Effects - GEO1}

The text and graphical output for GEO at 1 degree is displayed in the following graphs. For detailed graph descriptions on each graph see Section 5.1.

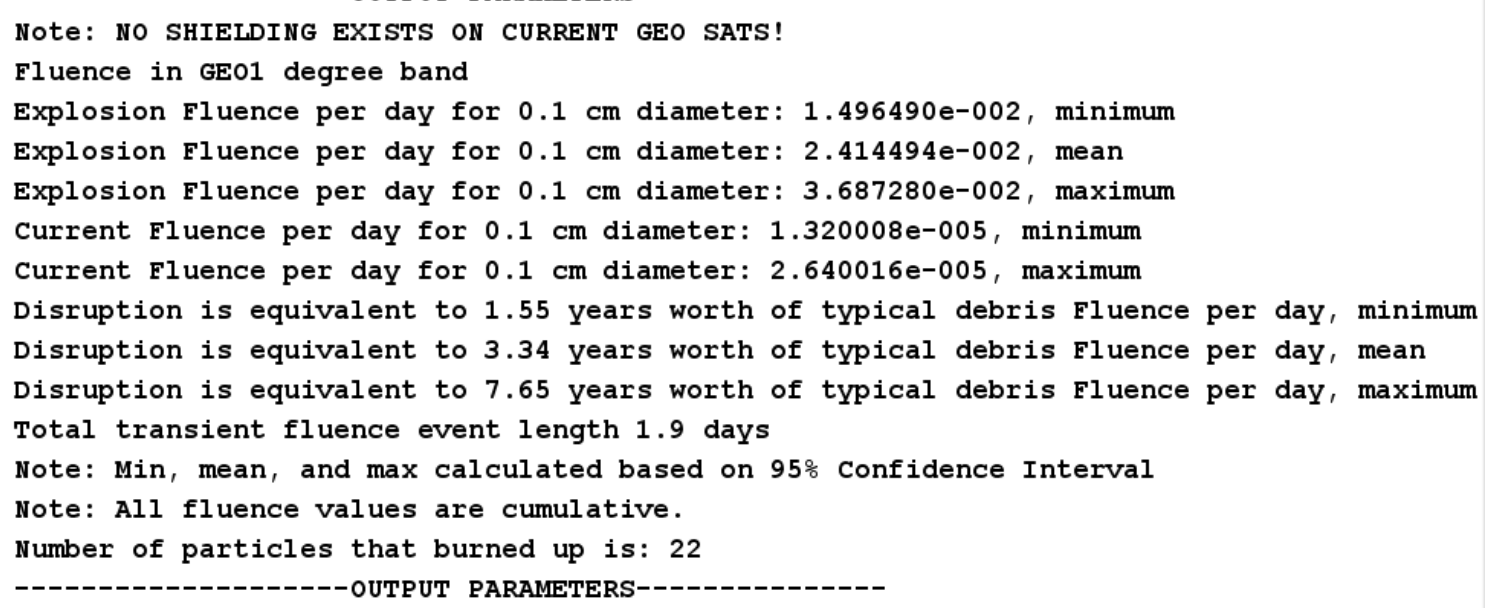

Figure 5.7: MATLAB Command Line Output 


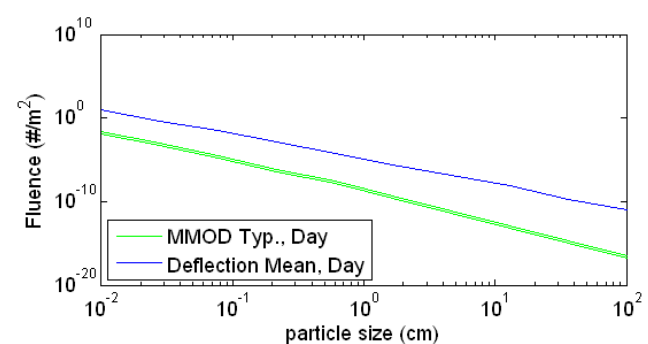

Figure 5.8: Mean MMOD Comparison

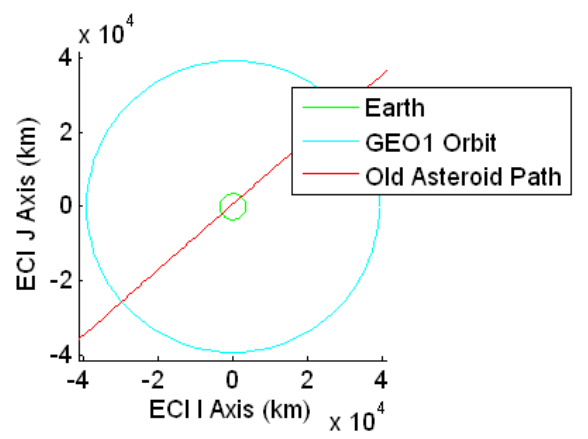

Figure 5.10: Original Asteroid Orbital Path Check

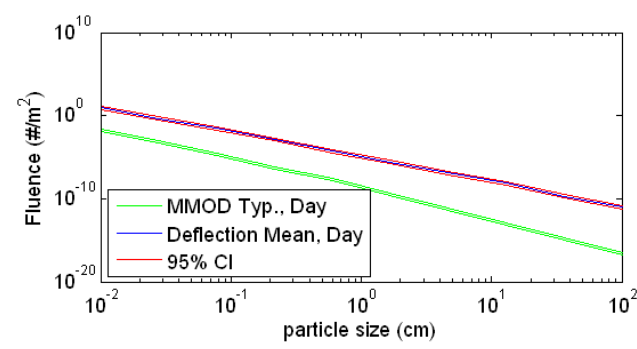

Figure 5.9: MMOD CI Comparison

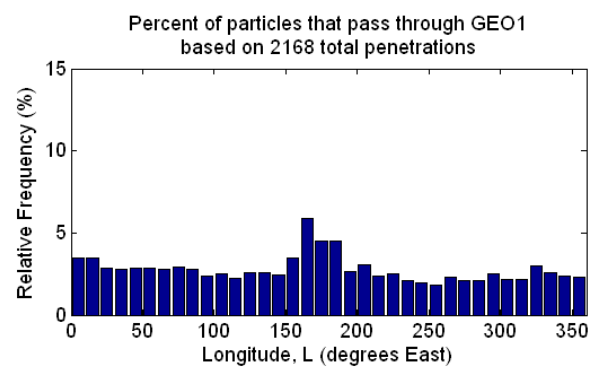

Figure 5.11: Longitude Bin Penetration Percentage

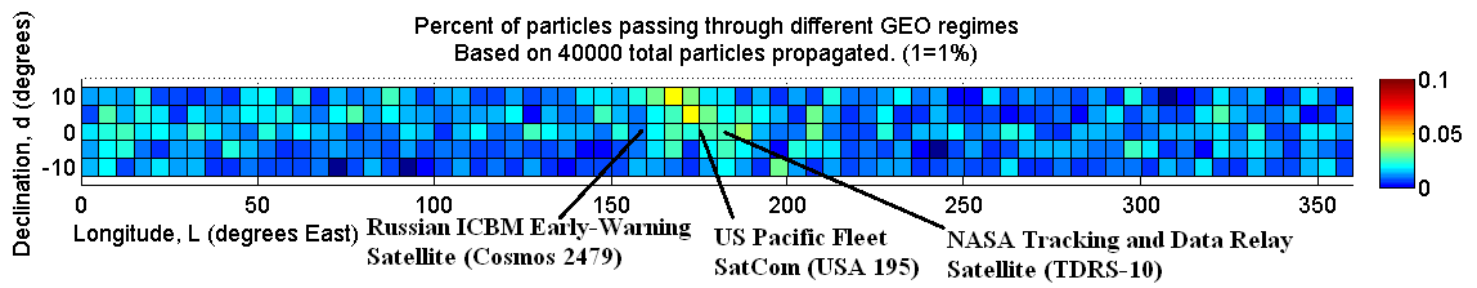

Figure 5.12: Heatmap of Penetrations of Critical Orbit With Satellite Locations 


\section{$5.2 \quad$ Fic2029-400}

The Fic2029-400 example is similar to the Fic2029-500 disruption. In this new example, the disruption of the same Fic2029 asteroid with a different magnitude payload of a $400 \mathrm{kt}$ nuclear warhead will be examined. The disruption date will be April 9th, 2029 and the input conditions to the program are listed in Table 5.4. To represent the difference in energy delivered to the asteroid, the change in Explosion Profile is changed to Weaver5. This profile represents a hydrodynamic study conducted by Dr. Robert Weaver at Los Alamos National Laboratories using the RAGE hydrodynamic code on the Cielo supercomputer. In this study, the largest remaining chunk size is not modified. This is an imperfect assumption because with a smaller explosion, it is expected that the largest remaining chunk will increase in size. However, since it is hard to qualify how much larger, the LRC size will be kept at $20 \mathrm{~m}$ diameter. 


\subsubsection{Input Parameters}

Table 5.4: Input Parameters, April 9th Disruption

\begin{tabular}{|c|c|c|}
\hline Explosion Date & {$[2029,4,9,0,0,0]$} & {$[$ year,mm,dd,hh,mm,ss] } \\
Observation Date & {$[2029,4,1,0,0,0]$} & Aear,mm,dd,hh,mm,ss] \\
\hline Position Earth (X) & $-9.803428546825277 \mathrm{E}-01$ & AU, Heliocentric \\
Position Earth (Y) & $-1.930901065107917 \mathrm{E}-01$ & AU, Heliocentric \\
Position Earth (Z) & $2.078772183457271 \mathrm{E}-05$ & AU, Heliocentric \\
\hline Velocity Earth (X) & $3.040684386769381 \mathrm{E}-03$ & AU/day, Heliocentric \\
Velocity Earth (Y) & $-1.693819813500764 \mathrm{E}-02$ & AU/day, Heliocentric \\
Velocity Earth (Z) & $8.062826223939135 \mathrm{E}-07$ & AU/day, Heliocentric \\
\hline Position Asteroid (X) & $-1.012505408225337 \mathrm{E}+00$ & AU, Heliocentric \\
Position Asteroid (Y) & $-2.212009168899217 \mathrm{E}-01$ & AU, Heliocentric \\
Position Asteroid (Z) & $-1.209751944049115 \mathrm{E}-02$ & AU, Heliocentric \\
\hline Velocity Asteroid (X) & $5.596108154750269 \mathrm{E}-03$ & AU/day, Heliocentric \\
Velocity Asteroid (Y) & $-1.481348469905512 \mathrm{E}-02$ & AU/day, Heliocentric \\
Velocity Asteroid (Z) & $9.217139513703808 \mathrm{E}-04$ & AU/day, Heliocentric \\
\hline Asteroid Mass & $3.51 \mathrm{E}+10$ & $\mathrm{~kg}$ \\
Asteroid Volume & $3.28736 \mathrm{E}+7$ & $\mathrm{~m}^{3}$ \\
Asteroid Density & 1.05 & $\mathrm{~g} / \mathrm{cm}^{3}$ \\
\hline Largest Remaining Chunk & 20 & meters \\
Explosion Profile & Weaver5.m & string \\
Fragments per Bin & 500 & number \\
\hline Propagation Loops & 8 & - \\
\# Fragment Bins & 10 & - \\
Total \# Fragments & 40,000 & - \\
\hline
\end{tabular}

\subsubsection{Results - April 9 Disruption}

Only the GEO1 fluence and the LEO425 fluence will be examined in these results. These critical orbits will be good indicators of the other critical orbit regimes. Other critical orbits will not be presented here.

Comparing the results of the Fic2029-400 and Fic2029-500 April 9th disruptions 
in Table 5.5 both the LEO425 and GEO1 fluence values are seen to overlap significantly, indicating that there is not much difference in overall fluence due to disruption at these two critical orbits. Furthermore, the heatmap graphs $(5.6 \& 8.66$ for LEO425 \& 5.12 \& 8.72 for GEO1) look very similar which supports this analysis. Thus, a cursory analysis after propagating 80,000 particles using two different veloc-

ity distributions (Weaver1 \& Weaver4) suggests that little difference in fluence will be experienced if a smaller nuclear explosion is used. However, this is assuming a constant Largest Chunk Size of 20 m diameter.

$\begin{array}{cccc}\text { Table 5.5: Results Comparison, } \mathbf{5 0 0} \mathbf{~ k t} \mathbf{~ v s} \mathbf{4 0 0} \mathbf{~ k t ,} \mathbf{2 0} \mathbf{~ m ~ L R C} \\ \text { Critical Orbit } & 500 \text { kt Disruption } & 400 \mathrm{kt} \text { Disruption } & \text { Units } \\ \text { LEO425 } & (1.3,3.8) & (1.1,2.4) & \text { days } \\ \text { GEO1 } & (1.5,7.7) & (3.1,12.5) & \text { years }\end{array}$

If the LRC increases by 10 meters in the $400 \mathrm{kt}$ detonation, a change in the resulting fluence is seen (see Table 5.6). With a $30 \mathrm{~m}$ diameter LRC with a 400 kt explosion, the resulting CI fluence does not at all overlap with the $500 \mathrm{kt}$ CI fluence. Thus it can be assumed that the means of the two explosions are different. Furthermore, it is expected that the 400kt explosion will cause 2.6 to 40.3 years of fluence more than the $500 \mathrm{kt}$ explosion based on the aforementioned assumptions. Because of this potentially large difference, it is essential that future studies analyze very carefully the LRC parameter. 
Table 5.6: Results Comparison, $500 \mathrm{kt} 20 \mathrm{~m} \mathrm{LRC} \mathrm{vs} 400 \mathrm{kt} 30 \mathrm{~m} \mathrm{LRC,}$ Critical Orbit $500 \mathrm{kt} \mathrm{w} / 20 \mathrm{~m} \mathrm{LRC} 400 \mathrm{kt} \mathrm{w} / 30 \mathrm{~m} \mathrm{LCR}$ Units

$\begin{array}{cccc}\text { LEO425 } & (1.3,3.8) & (11.3,24.1) & \text { days } \\ \text { GEO1 } & (1.5,7.7) & (10.3,41.8) & \text { years }\end{array}$

Tables $5.5 \& 5.6$ were constructed from results date found in the Appendix (Section 8.2).

Lastly, it is important to note that the difference in event duration between the two studies is very small (see Table 5.7). The event duration (event length) is orbit dependent and is the time difference between the first and last piercing of the critical orbit. The smaller explosion has a slightly shorter event duration. This is contrasted by later results using the Fic2029-600 example.

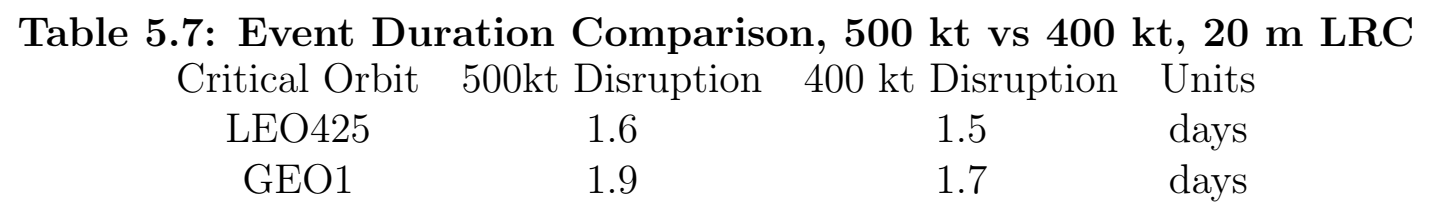

\section{$5.3 \quad$ Fic2029-600}

Similar to the previous two examples, the Fic2029-600 example will focus on disrupting the same Fic2029 asteroid on April 9th with a different strength of nuclear warhead: $600 \mathrm{kt}$. This involves changing the input parameters to use the Weaver4 explosion profile. The input parameters are contained in Table 5.8. In this study, the 
LRC size is not modified. This is an imperfect assumption as with a larger explosion, it is expected that the largest remaining chunk size will decrease in size. However, since it is hard to qualify how much smaller, the LRC size will remain unchanged at $20 \mathrm{~m}$ diameter.

\subsubsection{Input Parameters}

Table 5.8: Input Parameters, April 9th Disruption

\begin{tabular}{|c|c|c|}
\hline Explosion Date & {$[2029,4,9,0,0,0]$} & {$[$ year,mm,dd,hh,mm,ss] } \\
Observation Date & {$[2029,4,1,0,0,0]$} & [year,mm,dd,hh,mm,ss] \\
\hline Position Earth (X) & $-9.803428546825277 \mathrm{E}-01$ & AU, Heliocentric \\
Position Earth (Y) & $-1.930901065107917 \mathrm{E}-01$ & AU, Heliocentric \\
Position Earth (Z) & $2.078772183457271 \mathrm{E}-05$ & AU, Heliocentric \\
\hline Velocity Earth (X) & $3.040684386769381 \mathrm{E}-03$ & $\mathrm{AU} /$ day, Heliocentric \\
Velocity Earth (Y) & $-1.693819813500764 \mathrm{E}-02$ & $\mathrm{AU} /$ day, Heliocentric \\
Velocity Earth (Z) & $8.062826223939135 \mathrm{E}-07$ & $\mathrm{AU} /$ day, Heliocentric \\
\hline Position Asteroid (X) & $-1.012505408225337 \mathrm{E}+00$ & AU, Heliocentric \\
Position Asteroid (Y) & $-2.212009168899217 \mathrm{E}-01$ & AU, Heliocentric \\
Position Asteroid (Z) & $-1.209751944049115 \mathrm{E}-02$ & AU, Heliocentric \\
\hline Velocity Asteroid (X) & $5.596108154750269 \mathrm{E}-03$ & $\mathrm{AU} / \mathrm{day}$, Heliocentric \\
Velocity Asteroid (Y) & $-1.481348469905512 \mathrm{E}-02$ & $\mathrm{AU} /$ day, Heliocentric \\
Velocity Asteroid (Z) & $9.217139513703808 \mathrm{E}-04$ & AU/day, Heliocentric \\
\hline Asteroid Mass & $3.51 \mathrm{E}+10$ & $\mathrm{~kg}$ \\
Asteroid Volume & $3.28736 \mathrm{E}+7$ & $\mathrm{~m}^{3}$ \\
Asteroid Density & 1.05 & $\mathrm{~g} / \mathrm{cm}^{3}$ \\
\hline Largest Remaining Chunk & 20 & meters \\
Explosion Profile & Weaver4.m & string \\
Fragments per Bin & 500 & number \\
\hline Propagation Loops & 10 & - \\
\# Fragment Bins & 10 & - \\
Total \# Fragments & 50,000 & - \\
\hline
\end{tabular}




\subsubsection{Results - April 9 Disruption}

Only the GEO1 fluence and the LEO425 fluence will be examined in these results. These critical orbits will be good indicators of the other critical orbit regimes. The data which details on these orbit regimes is available for download as well but will not be presented here.

With similar largest remaining chunk size of $20 \mathrm{~m}$, a $500 \mathrm{kt}$ disruption shows significantly less fluence than a $600 \mathrm{kt}$ disruption (Table 5.9). The fluence at LEO425 levels is still close to levels that make little difference to satellites. The GEO1 fluence levels are on the orders of years. While the values are not statistically different, it is important to note that the event duration differs significantly (see Table 5.10). The event duration (event length) is orbit dependent and is the time difference between the first and last piercing of the critical orbit. This value is specified in the MATLAB output (see Figures 5.1, 5.7, 8.73, \& 8.79). For the 500kt explosion the event duration is much shorter than for the $600 \mathrm{kt}$ explosion. This indicates that while the daily fuence levels are not statistically different, the 600kt explosion fluence lasts longer. This indicates that a larger explosion may be worse than a smaller explosion. However, this result is highly dependent on the $20 \mathrm{~m} \mathrm{LRC} \mathrm{size.}$ When assigning a slightly smaller LCR size of $15 \mathrm{~m}$ to the $600 \mathrm{kt}$ explosion, the event duration stays the same but the fluence per day drops significantly (see Table 5.11). This suggests that oversizing a warhead does not create ill effects in this particular example. Tables 5.9, 5.10, \& 5.11 were constructed from results date found in the Appendix (Section 8.3). 
Table 5.9: CI Comparison, 500 kt vs 600 kt, 20 m LRC Critical Orbit 500kt Disruption 600 kt Disruption Units

$\begin{array}{cccc}\text { LEO425 } & (1.3,3.8) & (3.5,9.2) & \text { days } \\ \text { GEO1 } & (1.5,7.7) & (1.1,4.4) & \text { years }\end{array}$

Table 5.10: Event Duration Comparison, $500 \mathrm{kt}$ vs $600 \mathrm{kt}, 20 \mathrm{~m} \mathrm{LRC}$ Critical Orbit 500kt Disruption 600 kt Disruption Units

$\begin{array}{cccc}\text { LEO425 } & 1.6 & 2.2 & \text { days } \\ \text { GEO1 } & 1.9 & 3.7 & \text { days }\end{array}$

Table 5.11: Results Comparison, $500 \mathrm{kt} 20 \mathrm{~m} \mathrm{LRC} \mathrm{vs} 400 \mathrm{kt} 15 \mathrm{~m}$ LRC Critical Orbit $500 \mathrm{kt} \mathrm{w} / 20 \mathrm{~m} \mathrm{LRC} 600 \mathrm{kt} \mathrm{w} / 15 \mathrm{~m} \mathrm{LCR}$ Units
LEO425
$(1.3,3.8)$
$(0.7,1.6)$
days
GEO1
$(1.5,7.7)$
$(0.4,1.9)$
years 


\section{Chapter 6}

\section{Conclusions}

Satellites are critical for global communications. However, they are vulnerable to changes in the space environment. To analyze the changes in Micrometeoroid and Orbital Debris (MMOD) fluence due to asteroid disruption with nuclear explosions, a novel framework is presented. This paper shows how to successfully implement the framework to combine hydrodynamic simulation results, orbital propagation, multidimensional optimization, and statistics to analyze the MMOD side effects of asteroid disruption. Example scenarios showed side effects in some critical orbits were on the order of 3.3 of years of typical fluence per day for 1.9 days. In layman's terms, the average satellite in orbit would experience more than six years worth of weathering over two days. This indicates that substantial damage to satellites and degraded performance is highly likely in the scenarios presented. It was also shown that by changing disruption parameters such as the disruption date, explosion energy, and largest remaining chunk size, the MMOD fluence in critical orbit regimes can be 
modified.

Disruption of an asteroid can cause a significant increase in the MMOD fluence in the near-Earth space environment. However, this transient fluence is highly scenario dependent. The resulting confidence intervals for the transient fluence will vary depending on the specific geometry of the problem. This is governed by the location of Earth, the Moon, as well as the Asteroid, the time the disruption takes place as well as the strength of the explosion. While the scenario examples explored in this paper indicated a high fluence in the GEO1 at 150 to 200 degrees East longitude bin, and low fluence in LEO425, this is not expected to be the case for all disruption scenarios. Every asteroid disruption will produce different disruption results. The framework presented is a tool that can be used to study all of these disruption scenarios.

While no known asteroid is currently threatening to impact Earth, it is still essential to develop the tools to evaluate asteroid mitigation. This will allow decision makers to have sufficient analysis capabilities to completely understand the asteroid mitigation problem. Should a threatening asteroid appear, destructive mitigation is the most easily fielded technology. The framework demonstrated here shows that there could be significant consequences to this type of mitigation effort due to the large number of particles created by pulverizing the asteroid. Other non-destructive mitigation techniques, like gravity tractors, are capable of altering the trajectory of a threatening asteroid without the creation of a debris cloud. Non-destructive mitigation will ensure the least amount of MMOD fluence in critical orbits and will preserve critical space-based infrastructure. However, to develop these technologies, 
several decades of investment and research is required. The framework presented in this paper helps to quantify the high consequences of relying on destructive mitigation techniques.

Humankind is challenged to respect the threat that asteroids pose to our existence. As a reactionary species, it is difficult to respond to problems that do not cause current hardship. However, for our continual survival, we must begin to tackle problems that happen every 1,000 or 10,000 years. Upwards of $70 \%$ of all life on Earth was wiped out during the K-T event 65 million years ago. This event, which was caused by an asteroid the size of Mount Everest, wiped out the dinosaurs. If humankind avoids developing technologies to mitigate asteroids, nature may yet demonstrate that brain size has only a marginal effect on long-term species survival. 


\section{Chapter 7}

\section{Future Work}

This framework was implemented in MATLAB which is an effective language for demonstration. However, the large JAVA overhead of the program suggests that streamlined versions of the framework should be implemented in programs capable of handling calculations expediently. Faster calculations can be accomplished using the programs CUDA-C, C, and Fortran.

The propagator presented here uses many approximations and closed-form solutions in lieu of direct integration to allow the program to be implemented in MATLAB. For the accuracy of future studies, it is recommended to use more accurate methods of determining the location of the Moon, and other planets. Additionally, the propagation should be conducted about the solar system barycenter to avoid additional errors associated with assuming a stationary Sun. Additional accuracy can be gained by including procession and nutation in the formulation of the ECEF 
to ECI coordinate system transformation.

Current MMOD fluence levels are calculated using older Kessler and Grun models (see Section 3.1.5). More accurate models, such as ORDEM3.0, should be used when available.

This framework creates the ability for future studies to attempt to minimize the transient fluence. This involves tweaking the explosion parameters and disruption date until the lowest fluence is found. This will most likely be found to correspond to a high-yield explosion, minimal Largest Remaining Chunk Size, for a disruption far outside of Earth's SOI.

Minimizing the debris fluence created from a disruption is important to maximize spacecraft survivability. As can be seen in the Fic2029-500 example (see Section 5.1), the dates of disruption can cause varying amounts of fluence to transit critical orbit regimes. The disruption on April 9th or April 11th does not significantly change the amount of LEO fluence. However, this disruption date difference may cause a significant difference in the GEO fluence. This can be seen through the significant upwards shift of the CI on the April 11th disruption date. Further analysis could narrow this CI to see if the true mean fluence of each disruption date significantly differs. This would require simulating thousands of more fragments.

The explosion energy and the resulting explosion velocity and largest remaining chunk size imparted to the asteroid are critical factors which can increase or decrease fluence levels. Comparing the Fic2029-400, Fic2029-500, and Fic2029-600 GEO1 results, an increase in fluence levels can be seen with lower disruption energies and 
a larger LRC size. Careful estimation of the LRC is required to ensure it is properly sized as this factor has a significant effect on the resulting fluence levels.

The files for the program are available for download as a zip file and were implemented in MATLAB R2011a. Please contact Dr. Kira Abercromby in the Aerospace Engineering department at Cal Poly, SLO for details. 


\section{Chapter 8}

\section{Appendix}

\subsection{Fic2029-500 Additional Critical Orbits}

\subsubsection{Results - April 9 Disruption}

For detailed graph descriptions on each graph see Section 5.1.

Side Effects - LEO $800 \mathrm{~km}$

The text and graphical output for LEO at $800 \mathrm{~km}$ is displayed in the following graphs: 


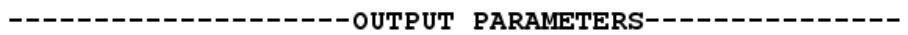

Note: Critical Diameter assumes LEO sats are shielded

Fluence in LEO800 degree band

Explosion Fluence per day for $1 \mathrm{~cm}$ diameter: 2.088078e-007, minimum

Explosion Fluence per day for $1 \mathrm{~cm}$ diameter: $3.378550 \mathrm{e}-007$, mean

Explosion Fluence per day for $1 \mathrm{~cm}$ diameter: 5.170918e-007, maximum

Current Fluence per day for $1 \mathrm{~cm}$ diameter: 2.681778e-007, minimum

Current Fluence per day for $1 \mathrm{~cm}$ diameter: 2.810591e-007, maximum

Disruption is equivalent to 0.7 days worth of typical debris Fluence per day, minimum

Disruption is equivalent to 1.2 days worth of typical debris Fluence per day, mean

Disruption is equivalent to 2 days worth of typical debris Fluence per day, maximum

Total transient fluence event length 1.6 days

Note: Min, mean, and max calculated based on 95\% Confidence Interval

Note: All fluence values are cumulative.

Number of particles that burned up is: 22

-----------------OOUTPUT PARAMETERS--------------

Figure 8.1: MATLAB Command Line Output

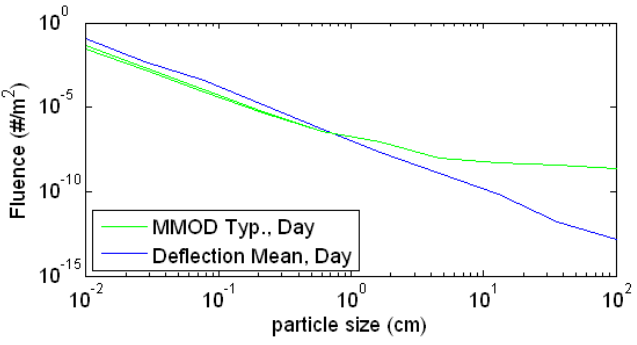

Figure 8.2: Mean MMOD Comparison

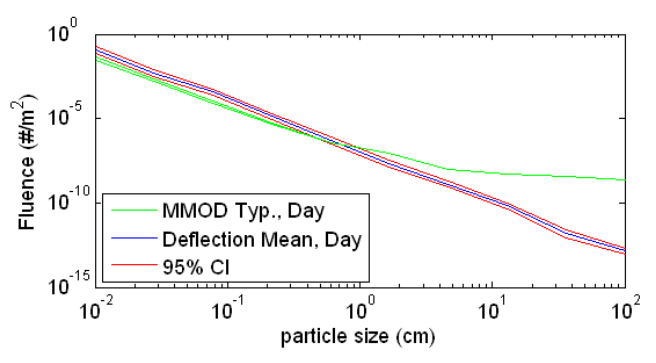

Figure 8.3: MMOD CI Comparison 


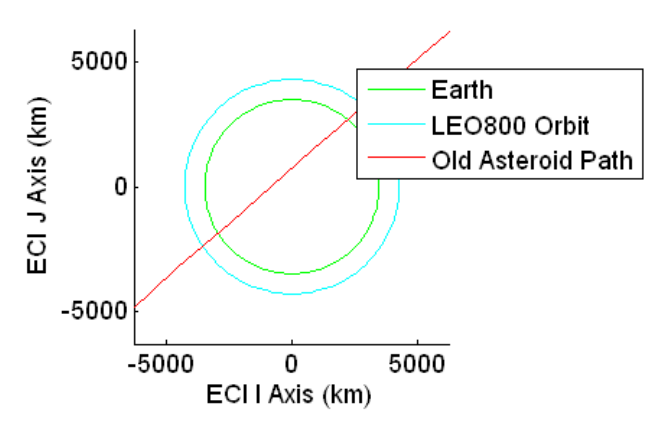

Figure 8.4: Original Asteroid Orbital Path Check

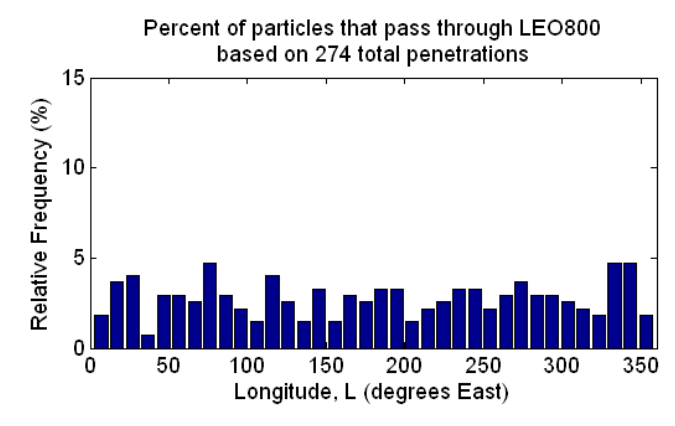

Figure 8.5: Longitude Bin Penetration Percentage

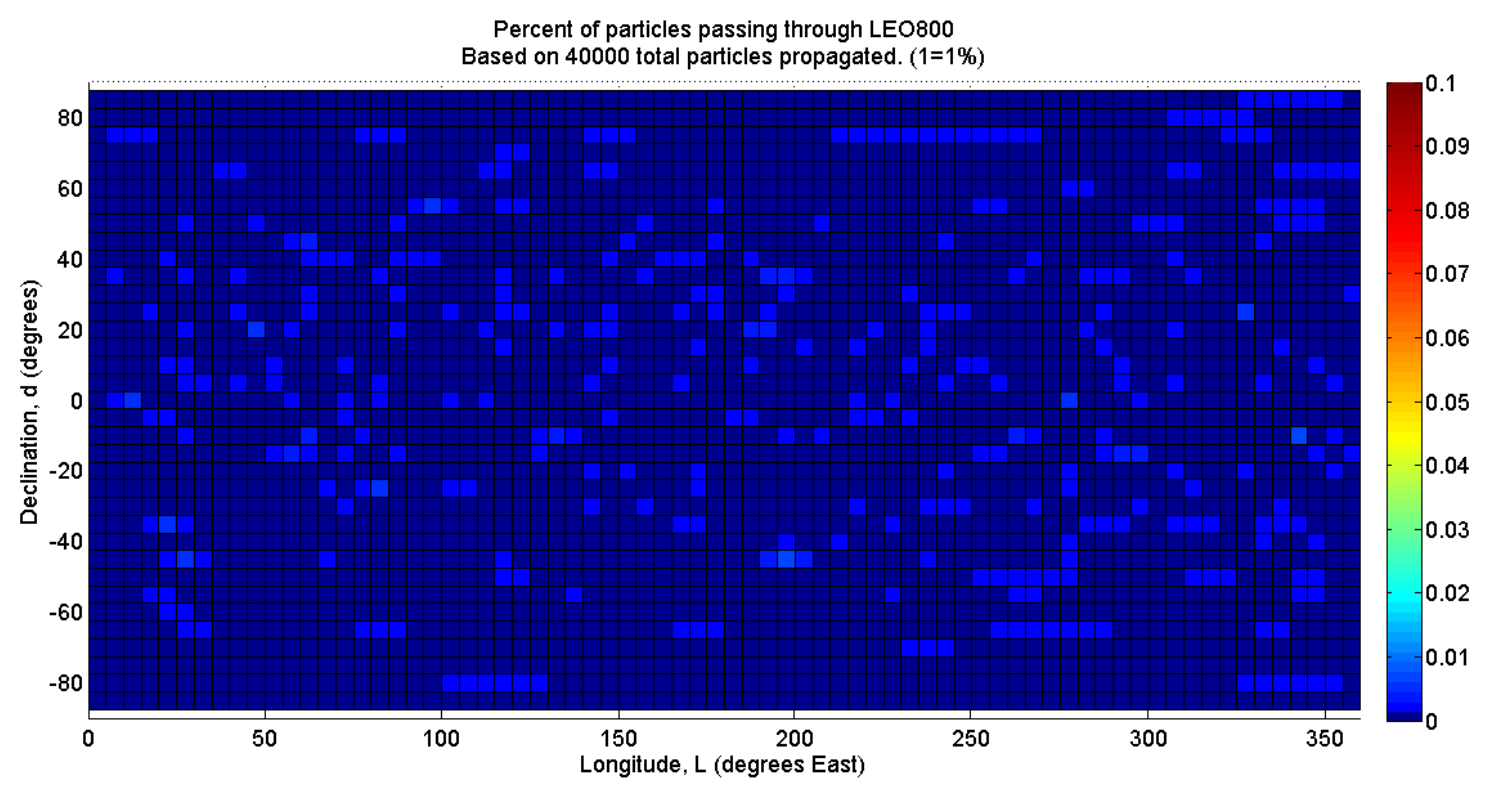

Figure 8.6: Heatmap of Penetrations of Critical Orbit 
Side Effects - LEO $1200 \mathrm{~km}$

The text and graphical output for LEO at $1200 \mathrm{~km}$ is displayed in the following graphs:

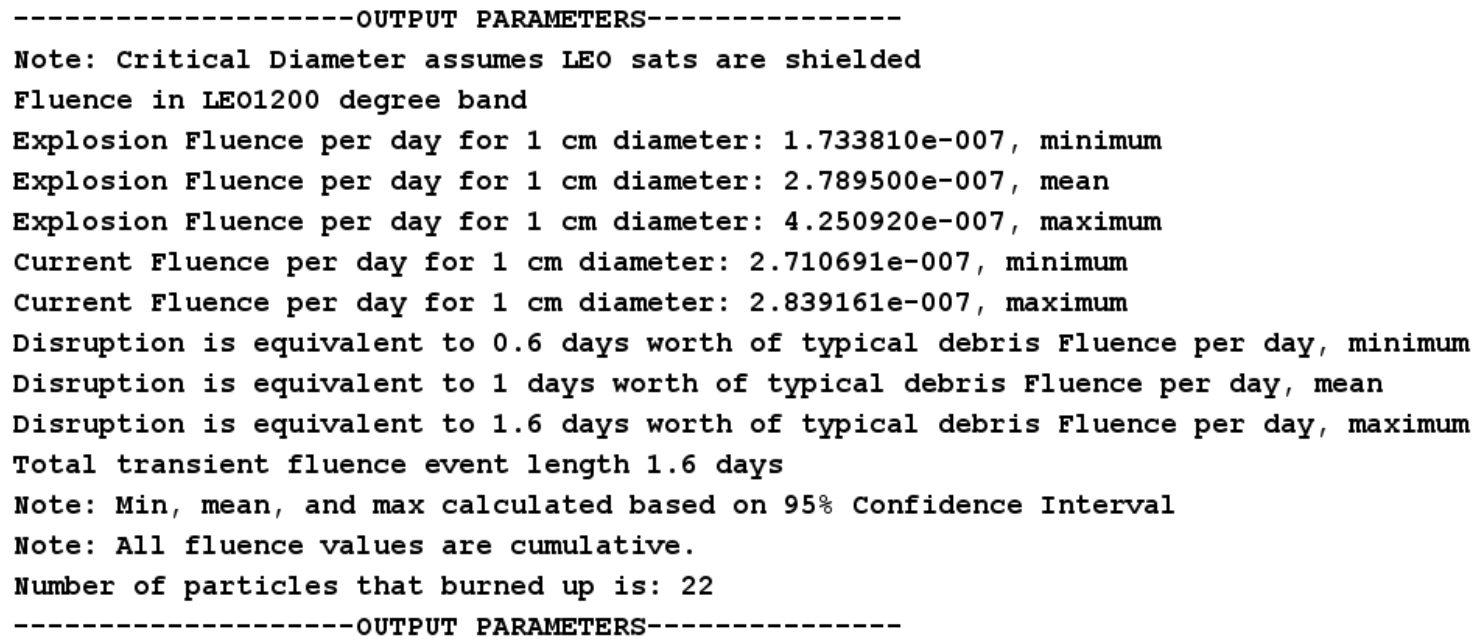

Figure 8.7: MATLAB Command Line Output

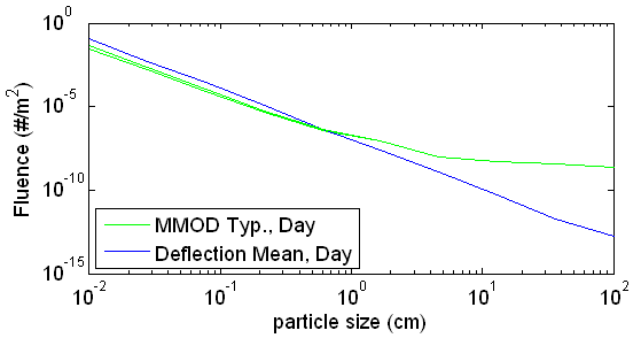

Figure 8.8: Mean MMOD Comparison

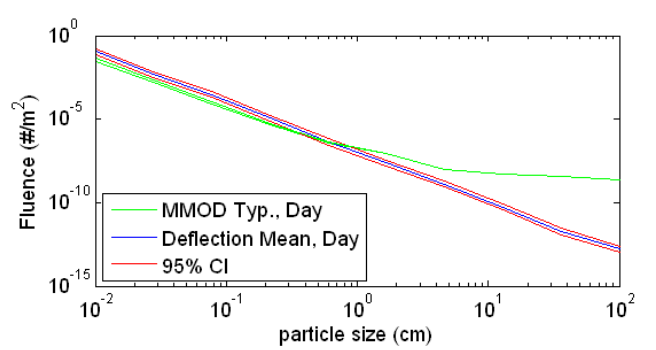

Figure 8.9: MMOD CI Comparison 


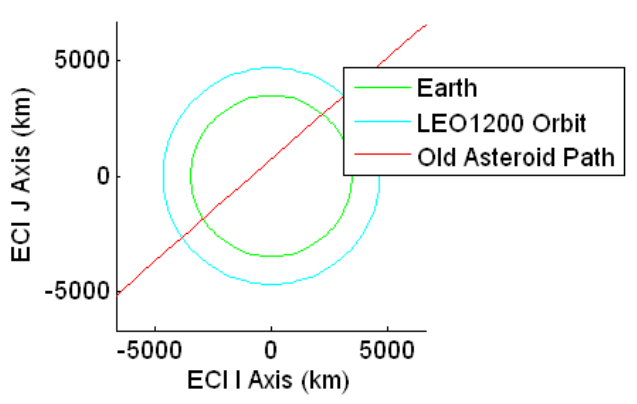

Figure 8.10: Original Asteroid Orbital Path Check

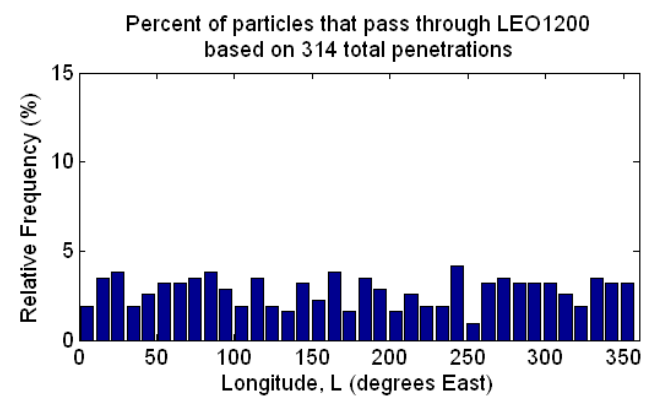

Figure 8.11: Longitude Bin Penetration Percentage

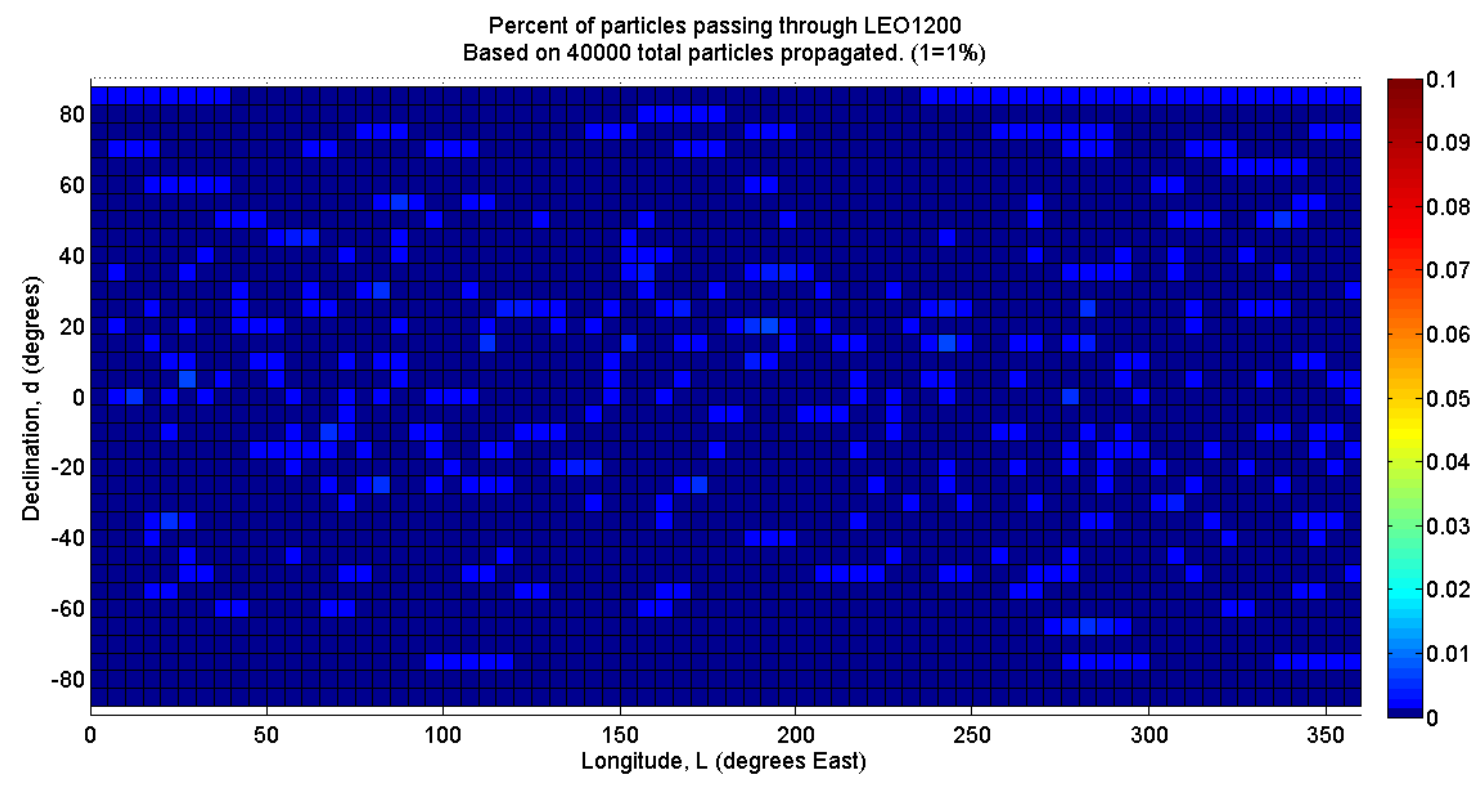

Figure 8.12: Heatmap of Penetrations of Critical Orbit 


\section{Side Effects - GEO4}

The text and graphical output for GEO at 4 degrees is displayed in the following graphs:

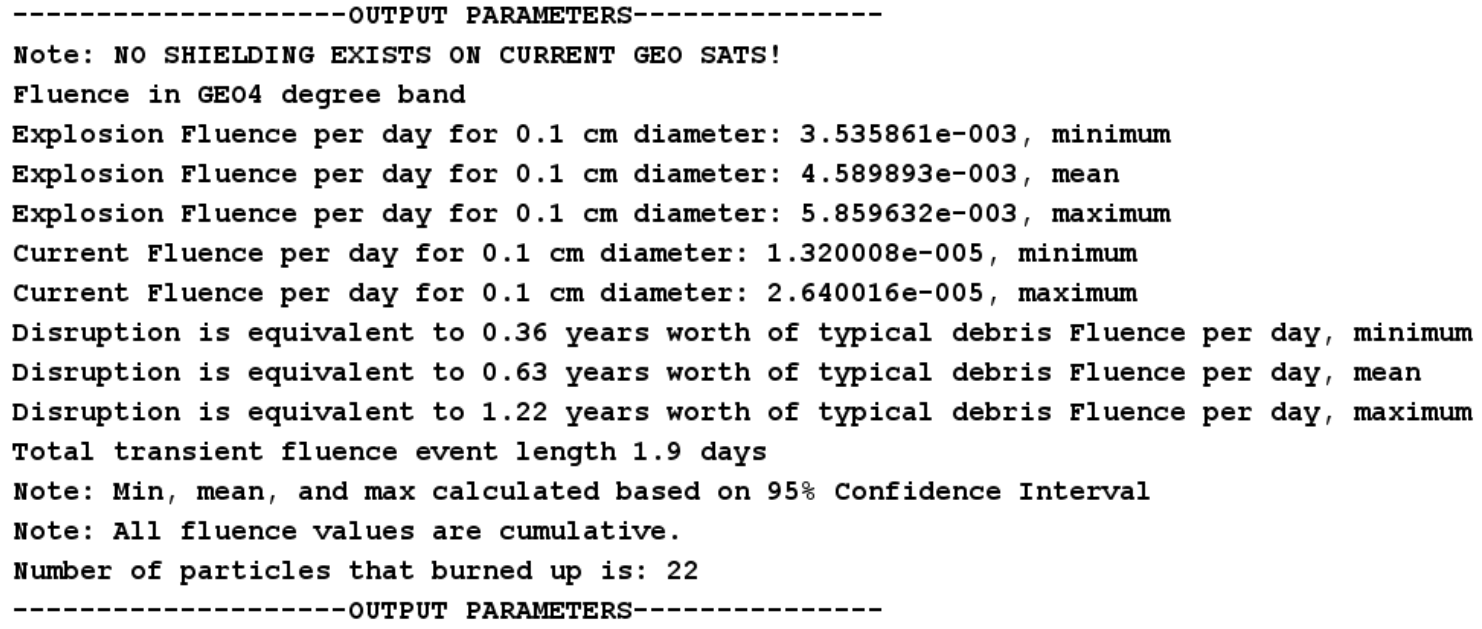

\section{Figure 8.13: MATLAB Command Line Output}

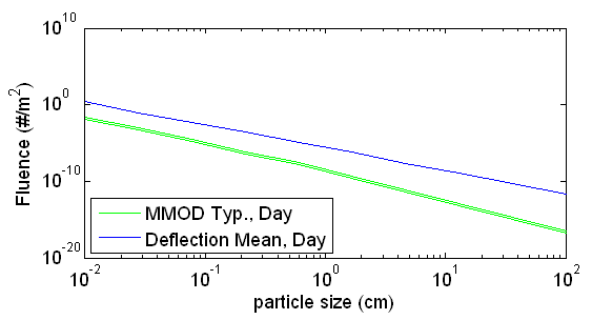

Figure 8.14: Mean MMOD Comparison

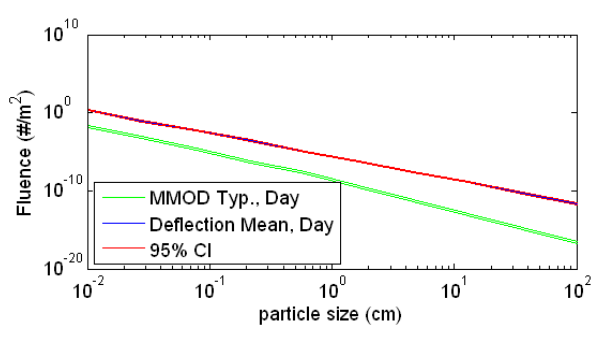

Figure 8.15: MMOD CI Comparison 


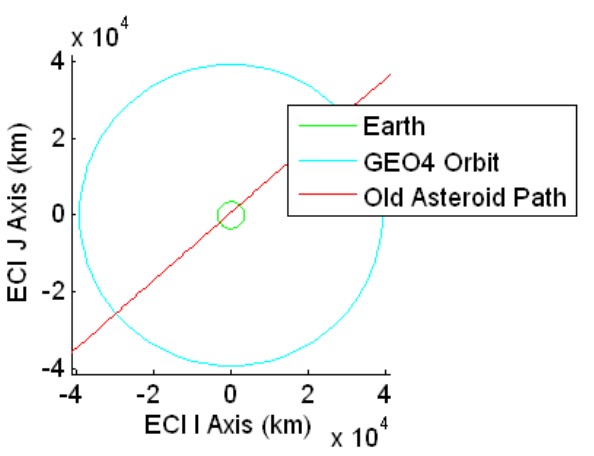

Figure 8.16: Original Asteroid Orbital Path Check

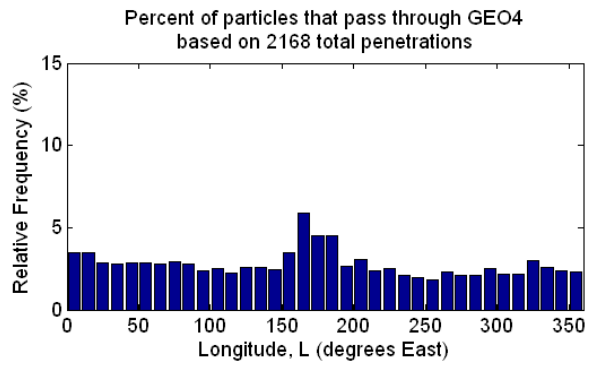

Figure 8.17: Longitude Bin Penetration Percentage

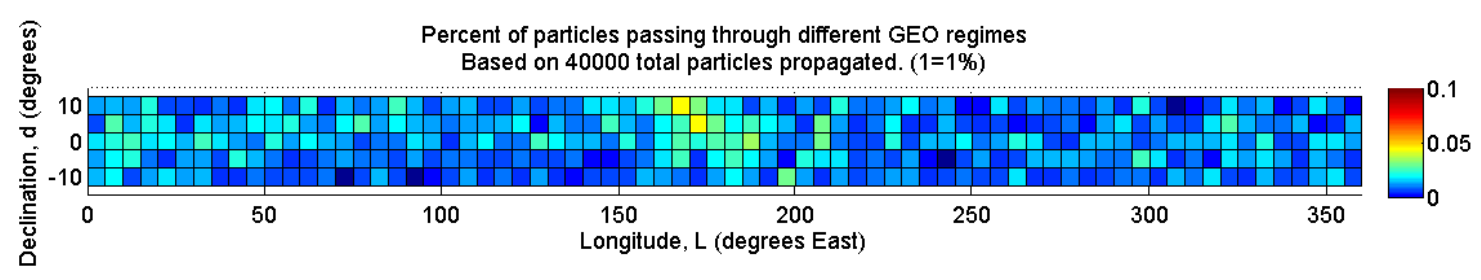

Figure 8.18: Heatmap of Penetrations of Critical Orbit 


\section{Side Effects - GEO15}

The text and graphical output for GEO at 15 degrees is displayed in the following graphs:

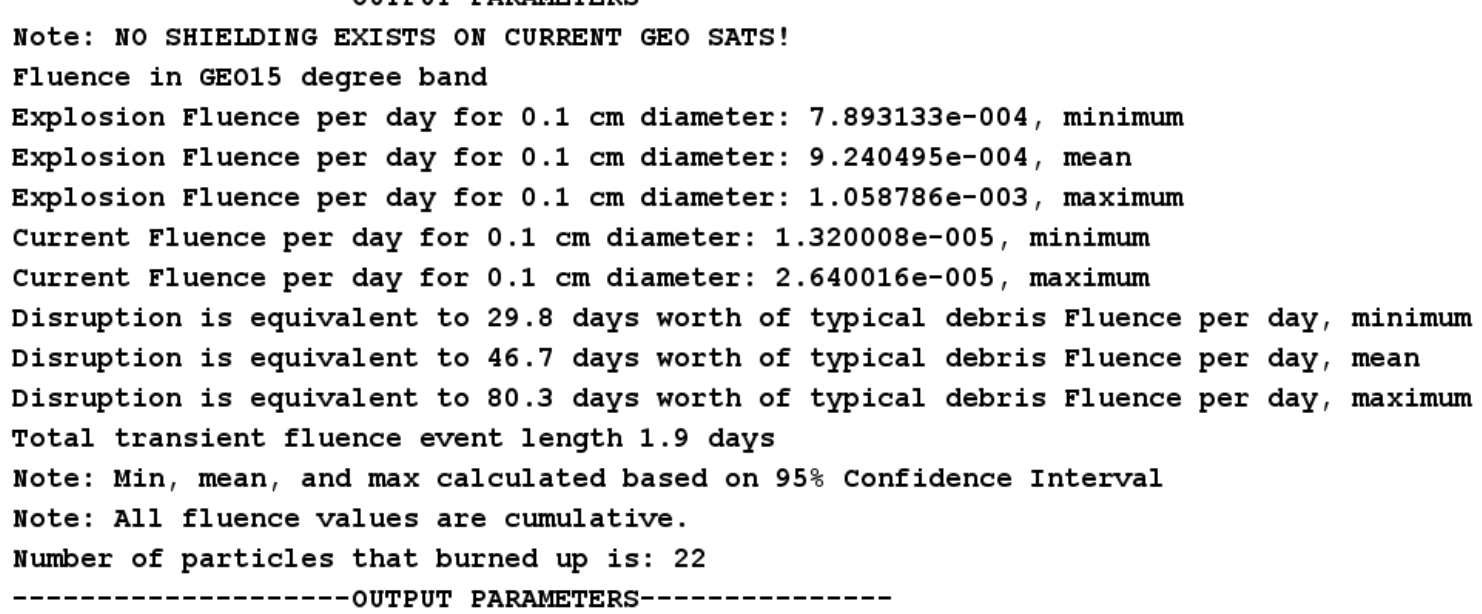

Figure 8.19: MATLAB Command Line Output

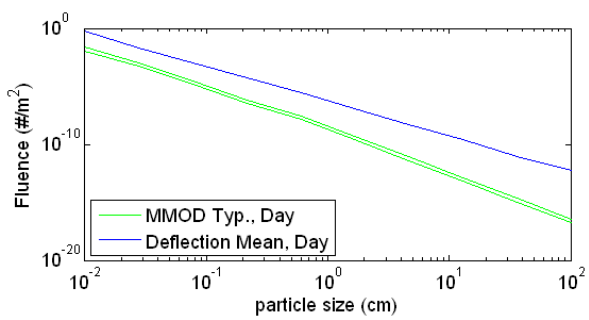

Figure 8.20: Mean MMOD Comparison

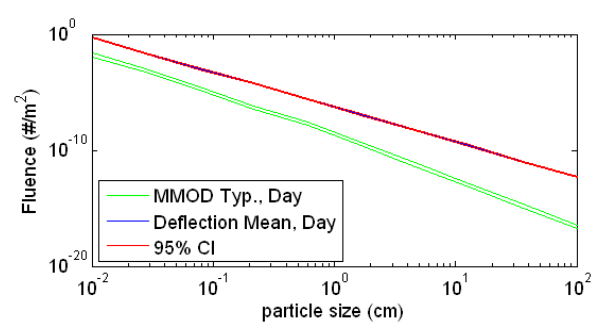

Figure 8.21: MMOD CI Comparison 


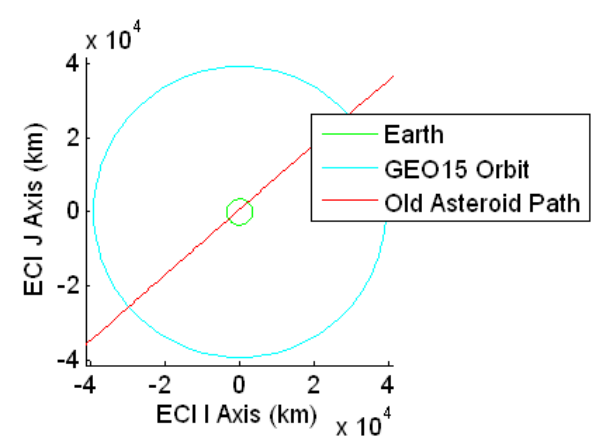

Figure 8.22: Original Asteroid Orbital Path Check

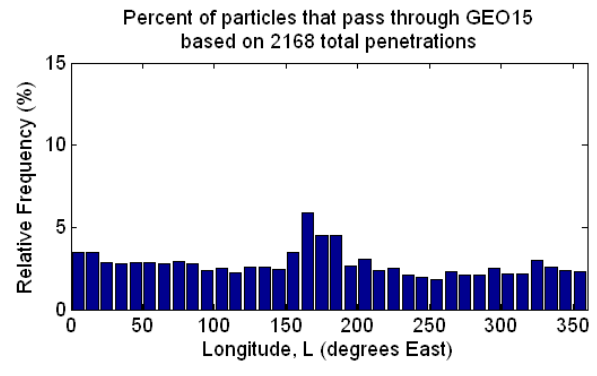

Figure 8.23: Longitude Bin Penetration Percentage

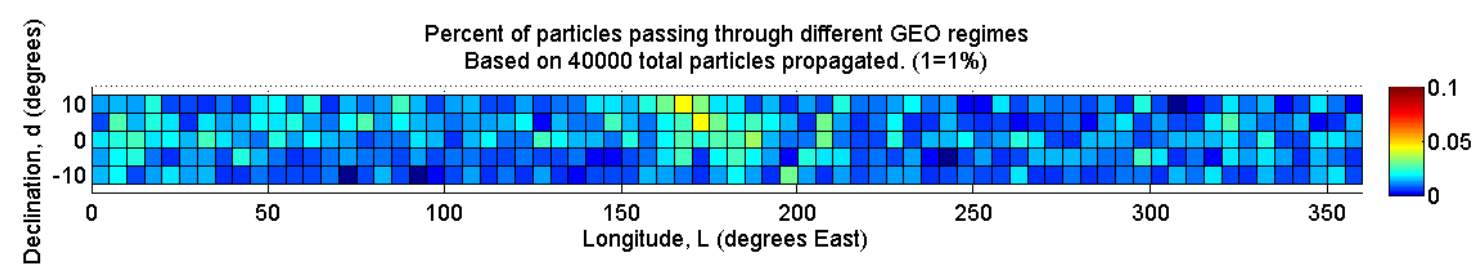

Figure 8.24: Heatmap of Penetrations of Critical Orbit

\subsubsection{Results - April 11 Disruption}

For detailed graph descriptions on each graph see Section 5.1.

Side Effects - LEO $425 \mathrm{~km}$

The text and graphical output for LEO at $425 \mathrm{~km}$ is displayed in the following graphs: 


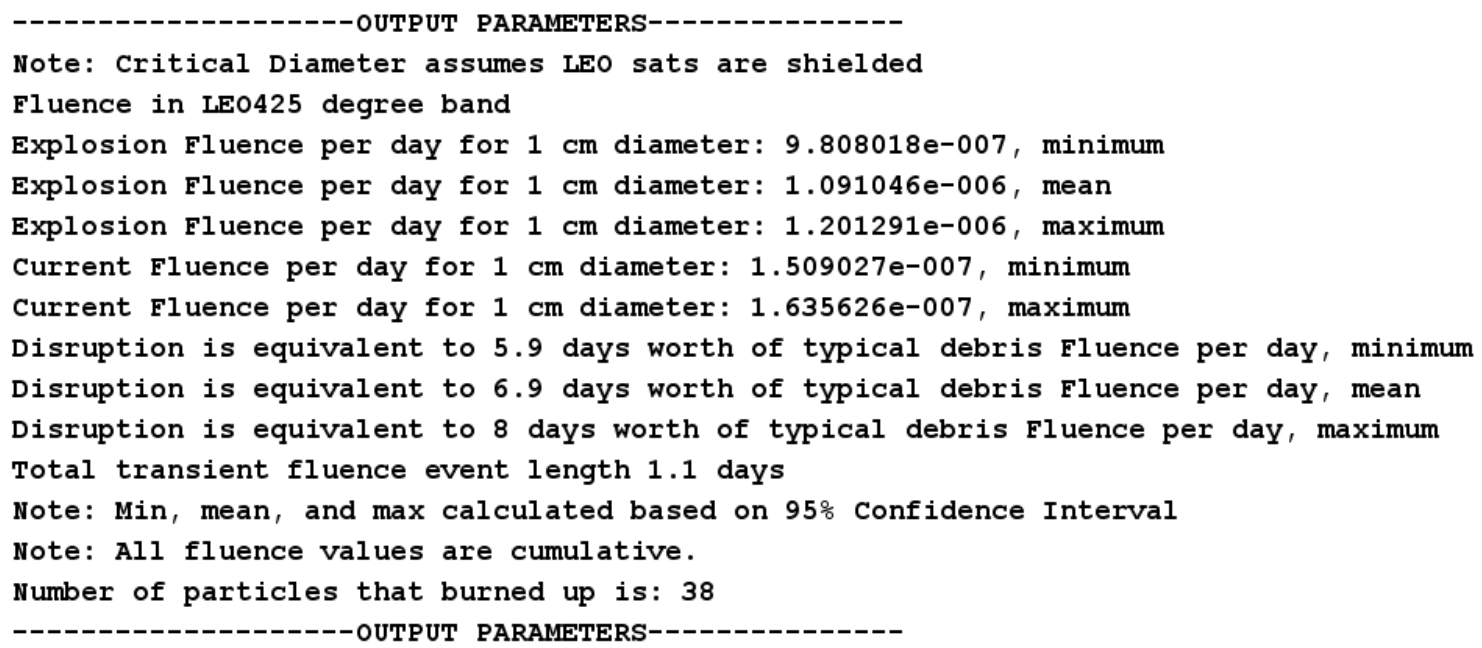

\section{Figure 8.25: MATLAB Command Line Output}

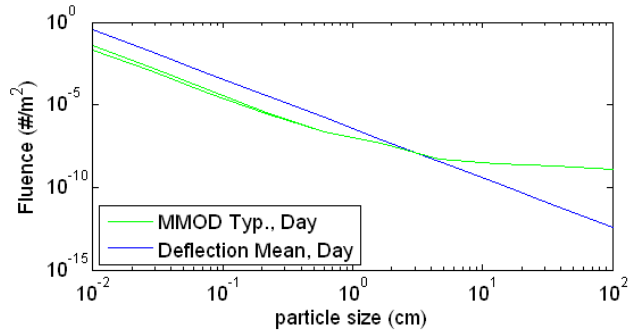

Figure 8.26: Mean MMOD Comparison

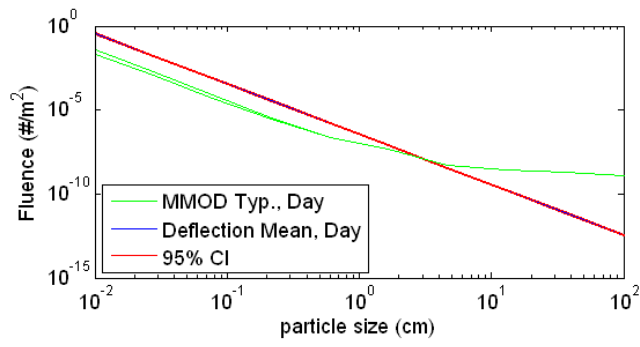

Figure 8.27: MMOD CI Comparison 


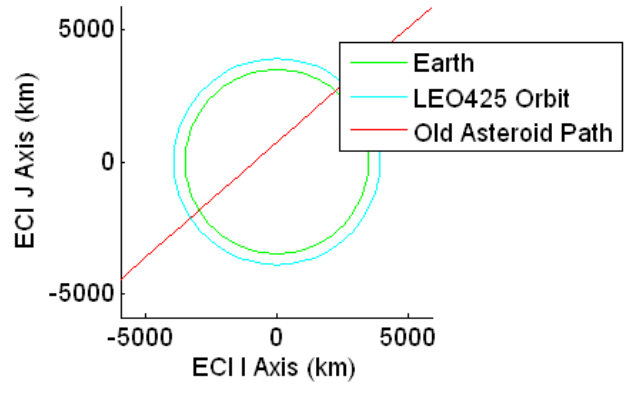

Figure 8.28: Original Asteroid Orbital Path Check

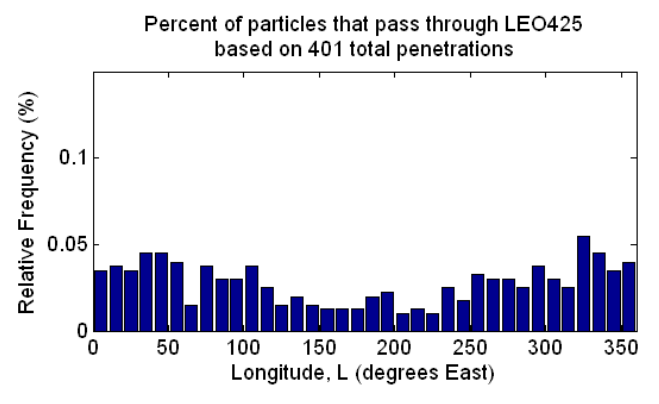

Figure 8.29: Longitude Bin Penetration Percentage

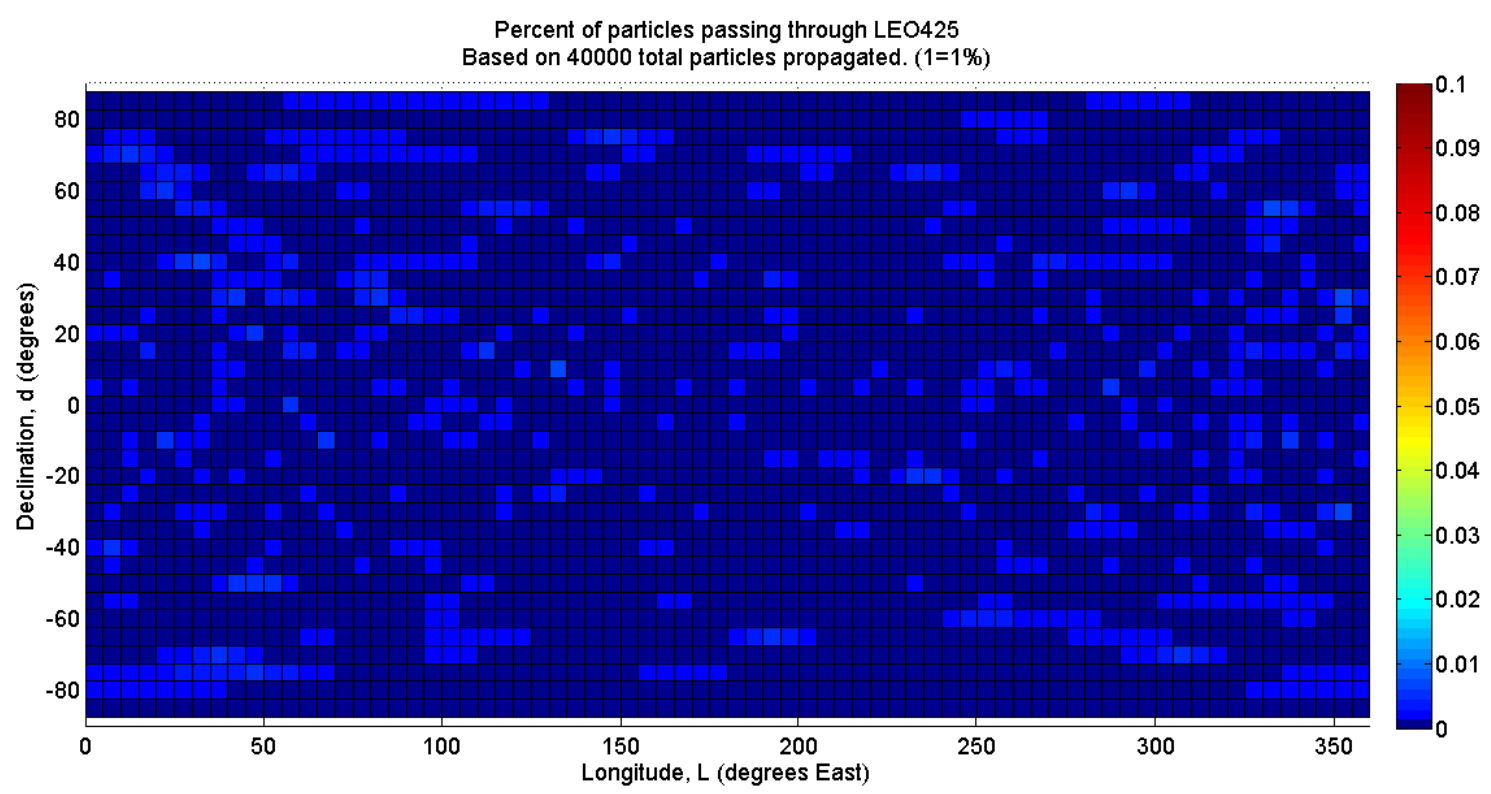

Figure 8.30: Heatmap of Penetrations of Critical Orbit 


\section{Side Effects - LEO 800 km}

The text and graphical output for LEO at $800 \mathrm{~km}$ is displayed in the following graphs:

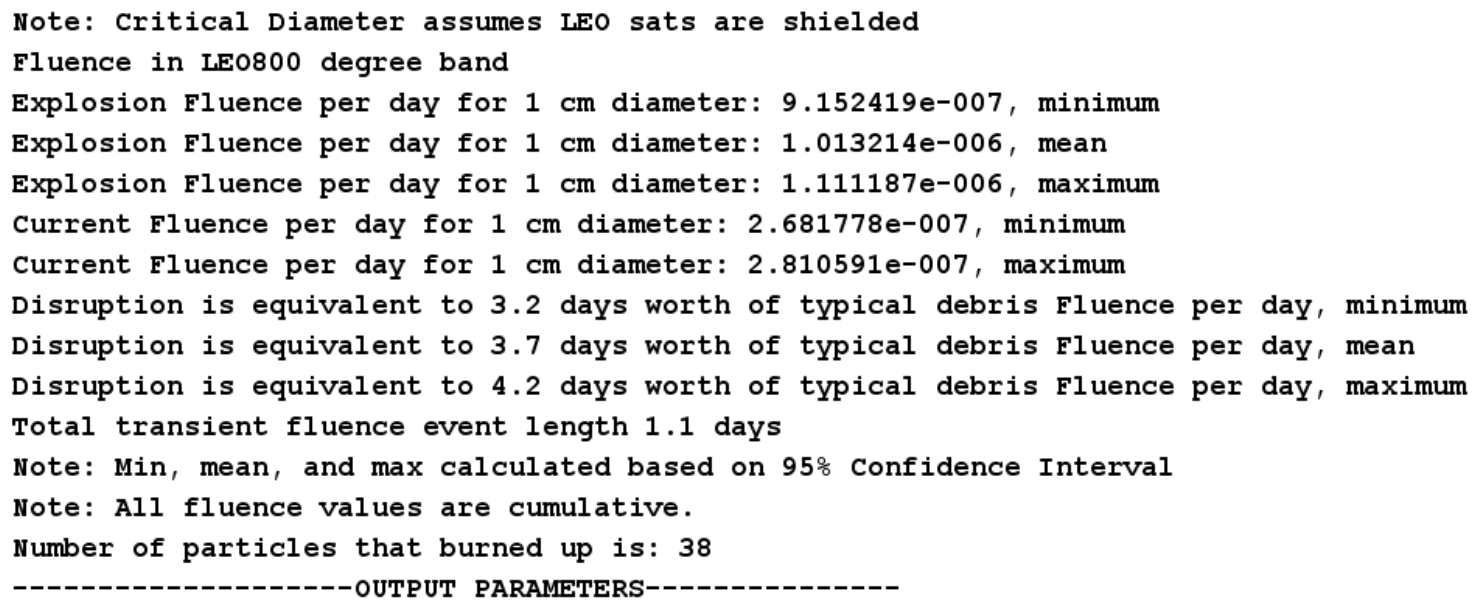

\section{Figure 8.31: MATLAB Command Line Output}

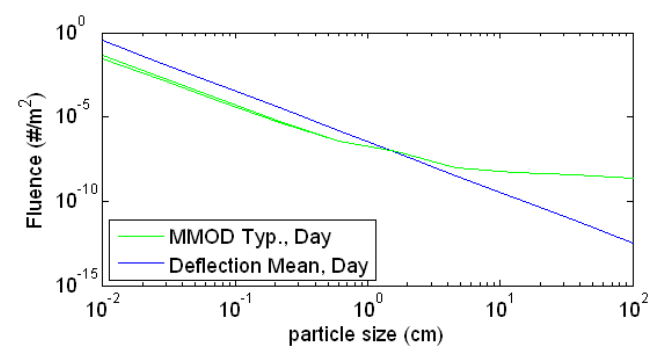

Figure 8.32: Mean MMOD Comparison

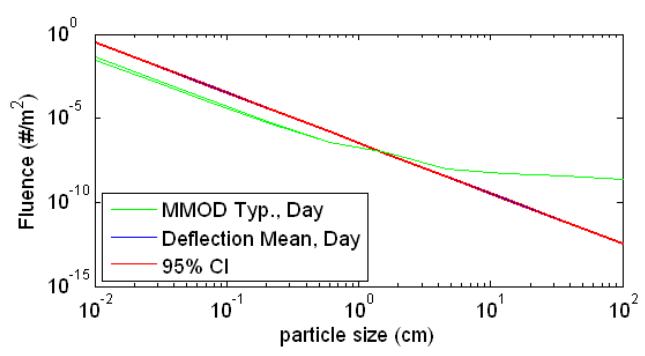

Figure 8.33: MMOD CI Comparison 


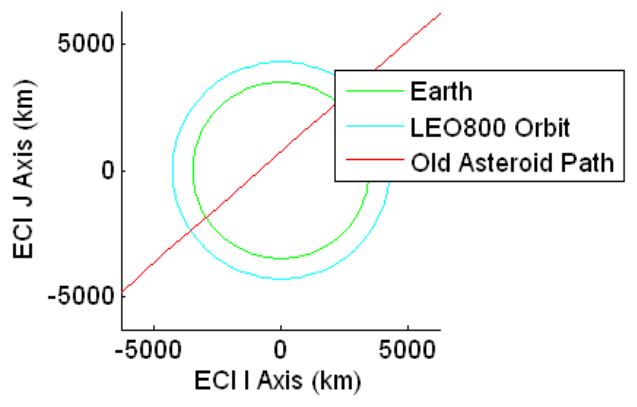

Figure 8.34: Original Asteroid Orbital Path Check

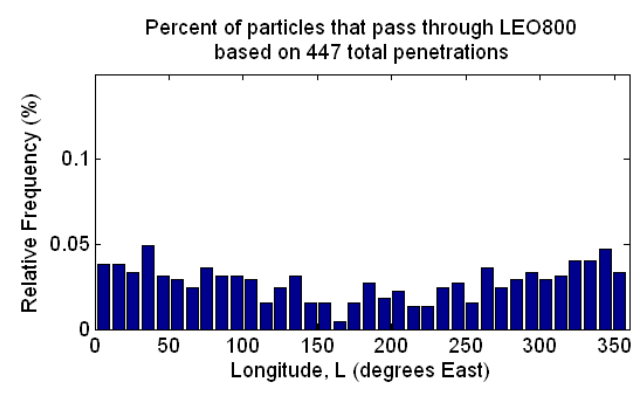

Figure 8.35: Longitude Bin Penetration Percentage

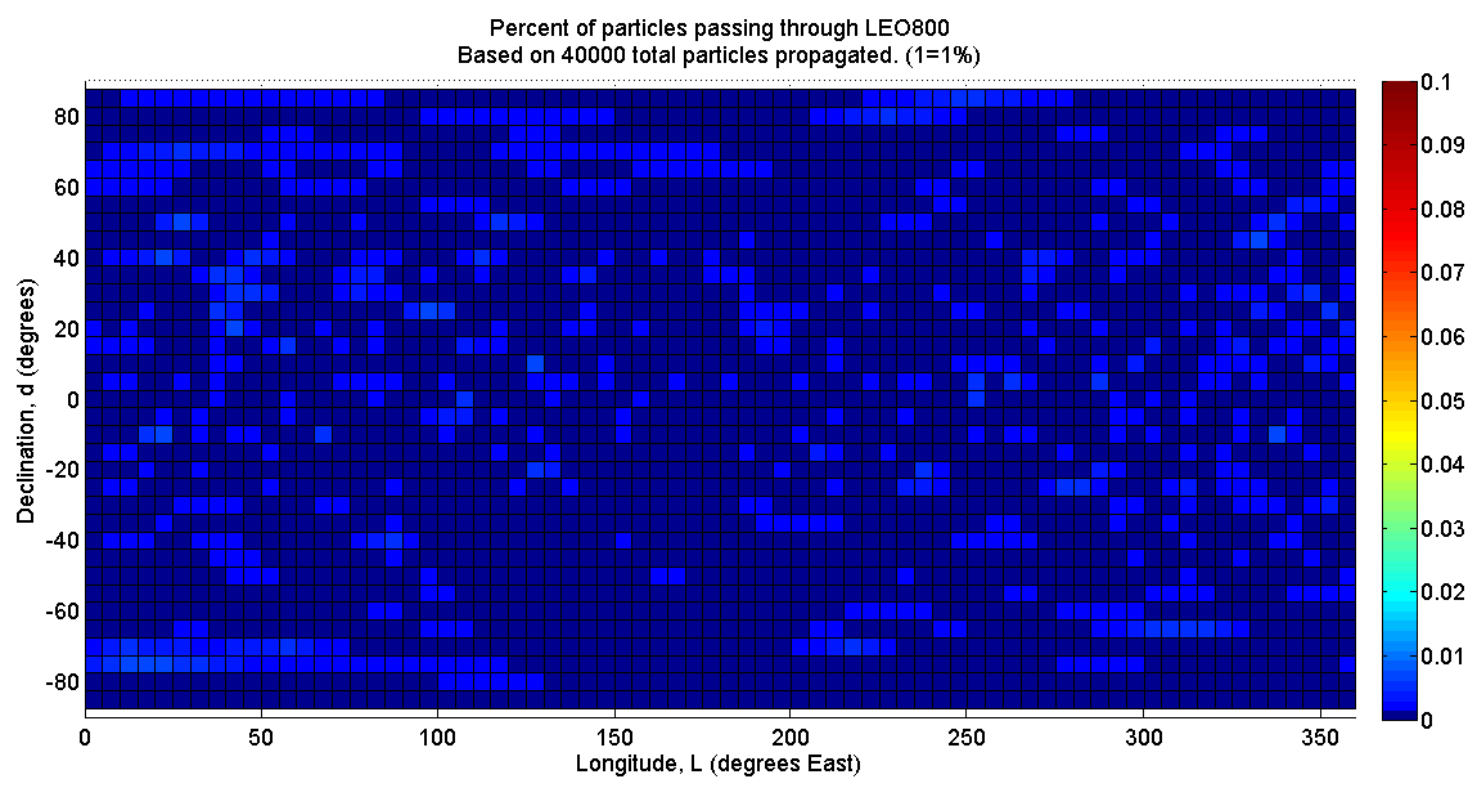

Figure 8.36: Heatmap of Penetrations of Critical Orbit 
Side Effects - LEO 1200 km

The text and graphical output for LEO at $1200 \mathrm{~km}$ is displayed in the following graphs:

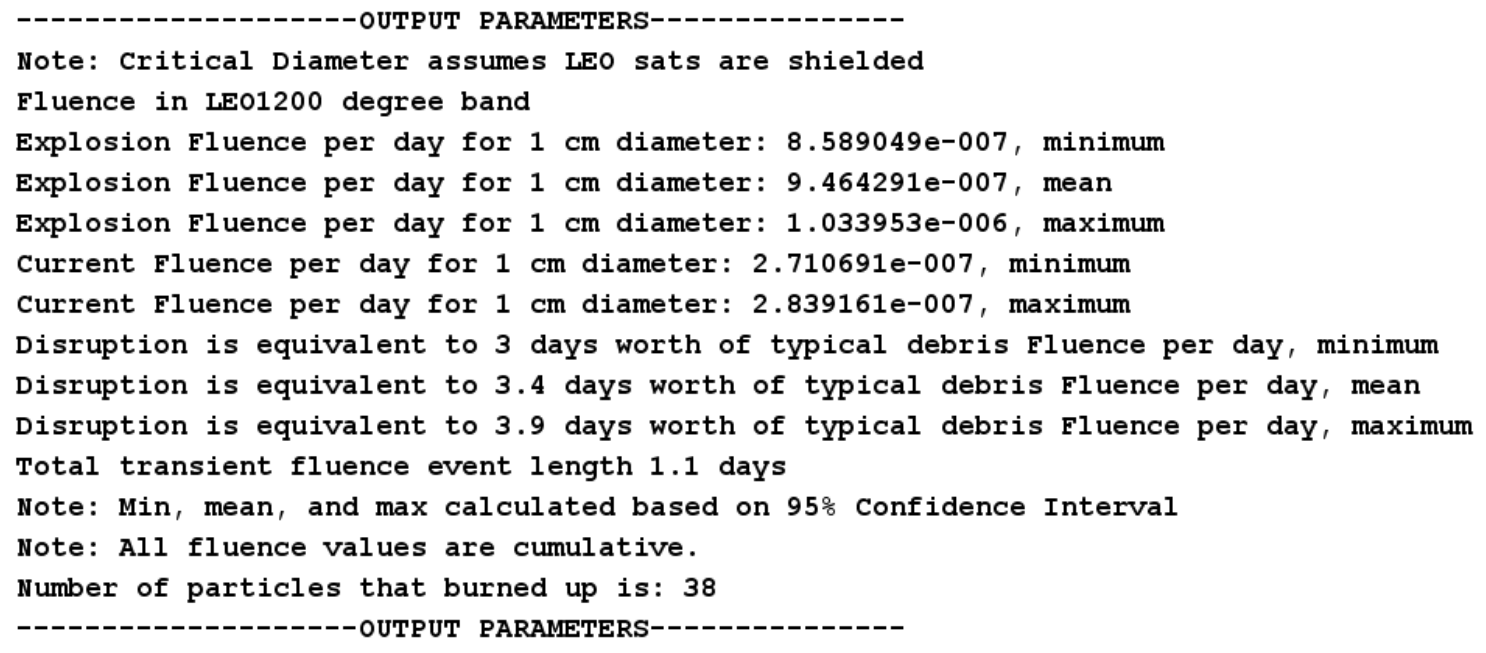

Figure 8.37: MATLAB Command Line Output

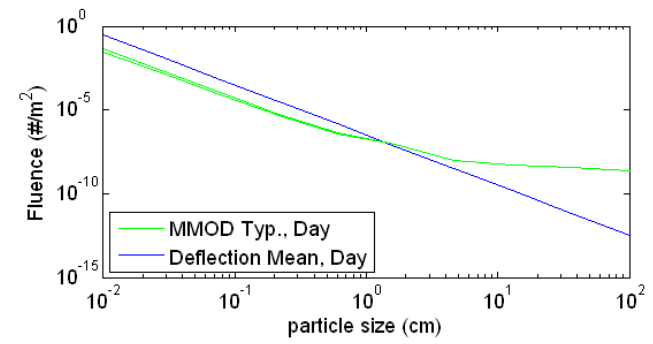

Figure 8.38: Mean MMOD Comparison

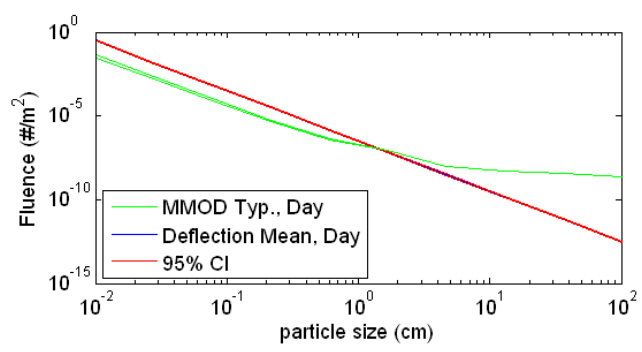

Figure 8.39: MMOD CI Comparison 


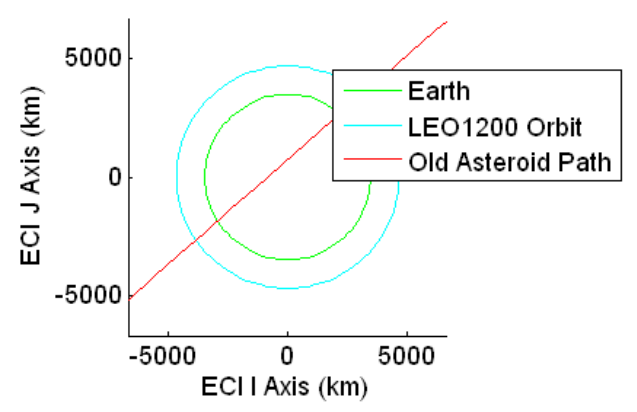

Figure 8.40: Original Asteroid Orbital Path Check

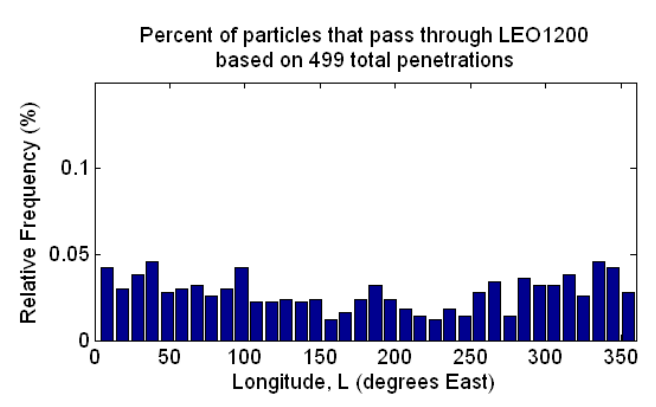

Figure 8.41: Longitude Bin Penetration Percentage

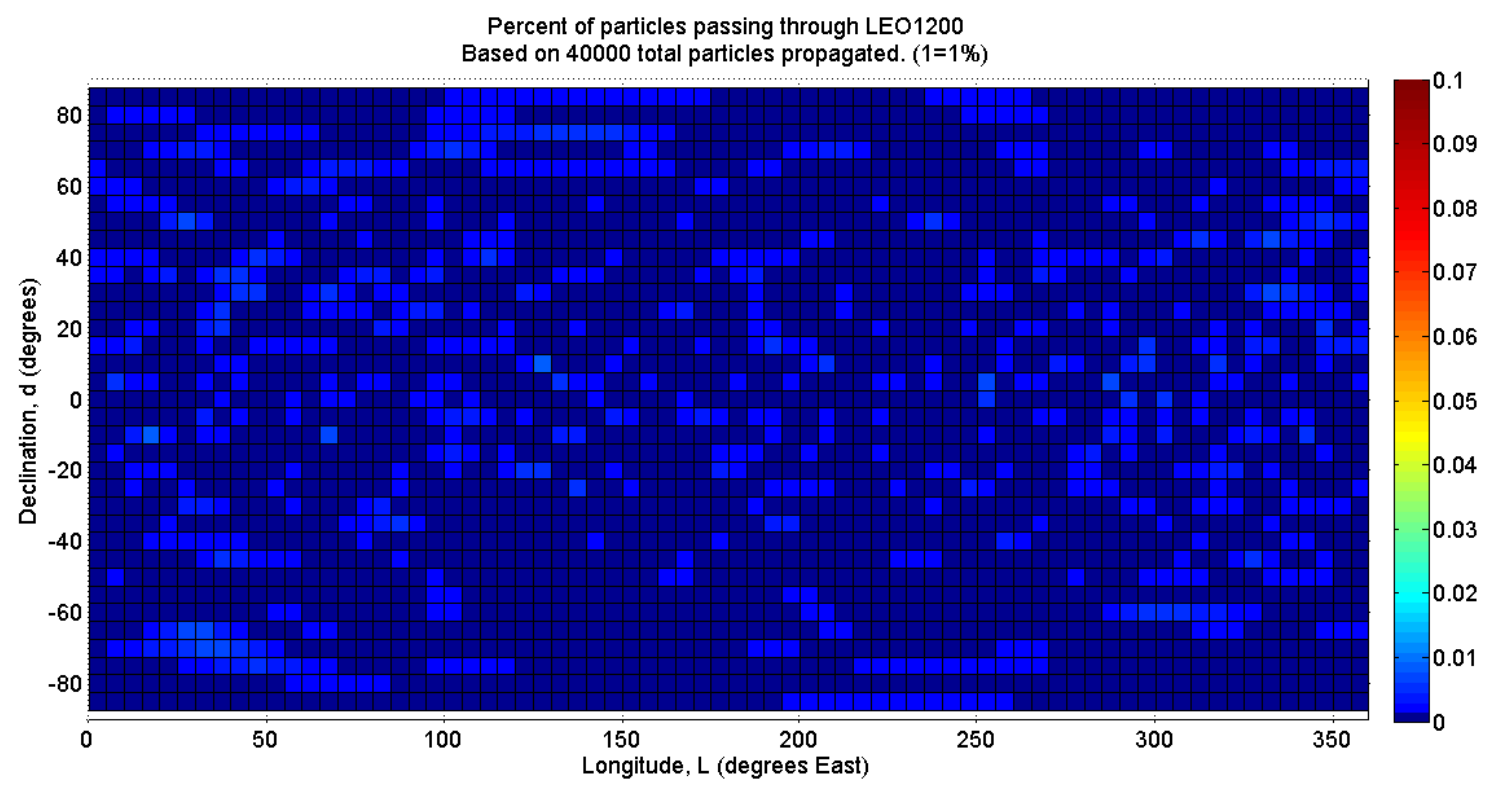

Figure 8.42: Heatmap of Penetrations of Critical Orbit 
Side Effects - GEO1

The text and graphical output for GEO at 1 degree is displayed in the following graphs:

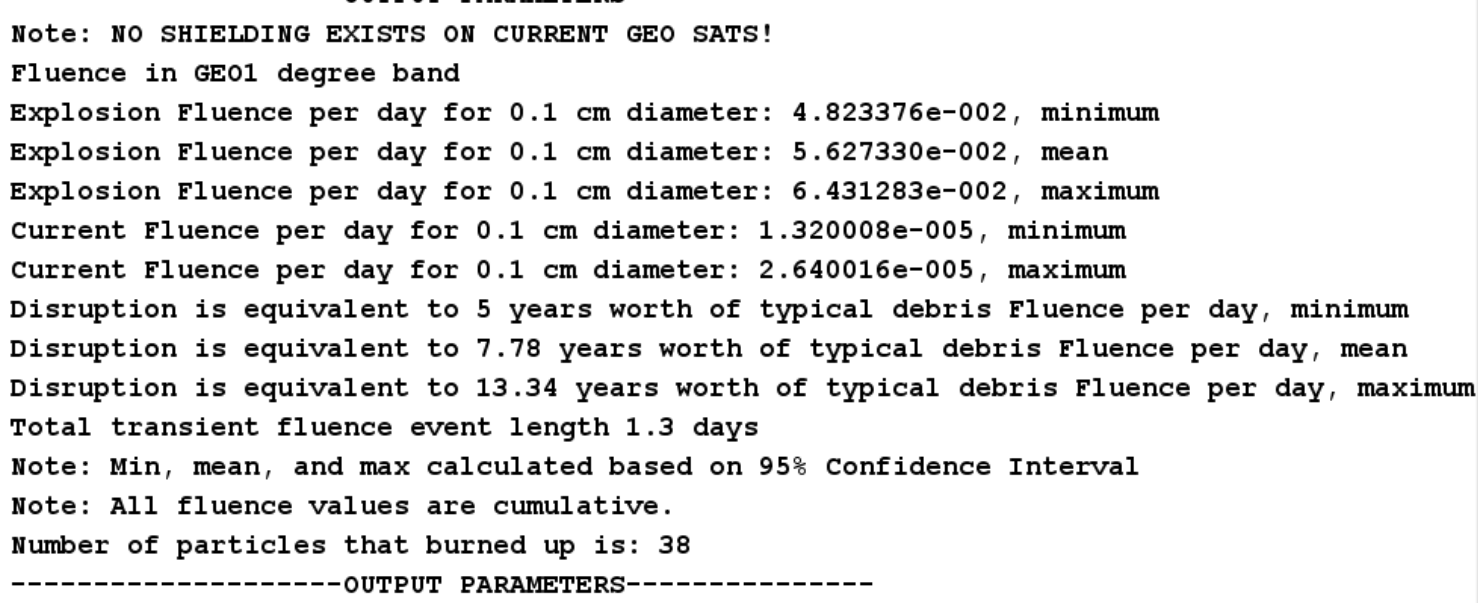

Figure 8.43: MATLAB Command Line Output

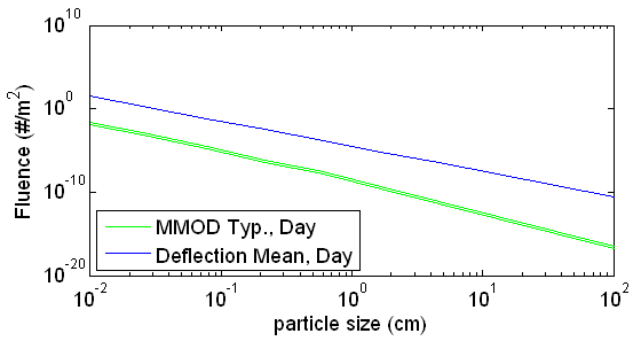

Figure 8.44: Mean MMOD Comparison

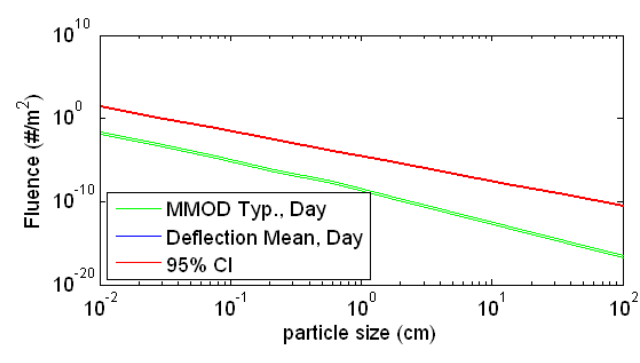

Figure 8.45: MMOD CI Comparison 


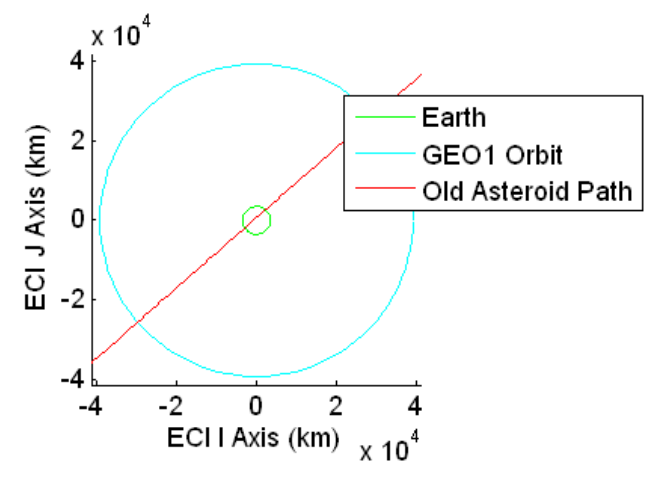

Figure 8.46: Original Asteroid Orbital Path Check

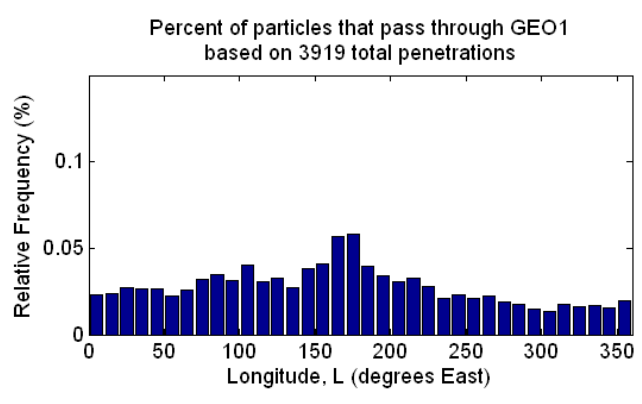

Figure 8.47: Longitude Bin Penetration Percentage

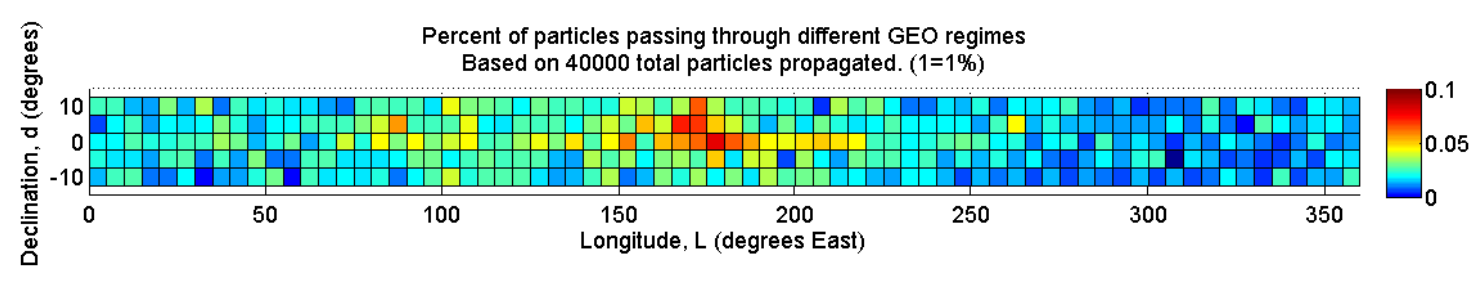

Figure 8.48: Heatmap of Penetrations of Critical Orbit 


\section{Side Effects - GEO4}

The text and graphical output for GEO at 4 degrees is displayed in the following graphs:

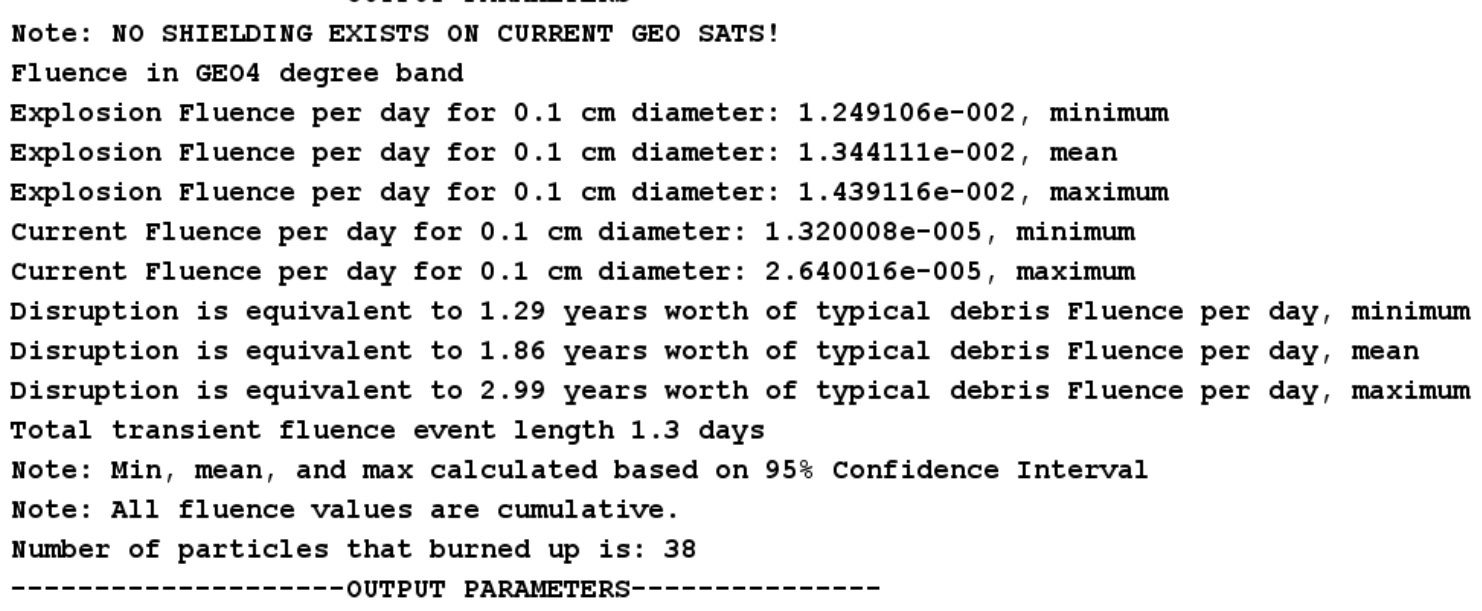

Figure 8.49: MATLAB Command Line Output

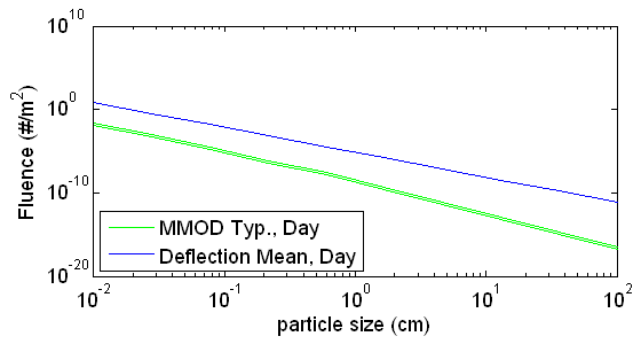

Figure 8.50: Mean MMOD Comparison

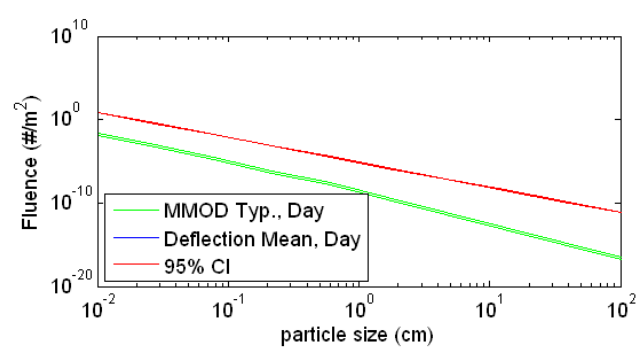

Figure 8.51: MMOD CI Comparison 


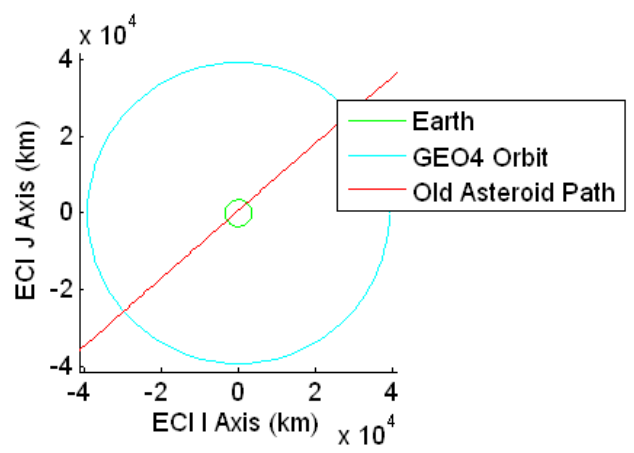

Figure 8.52: Original Asteroid Orbital Path Check

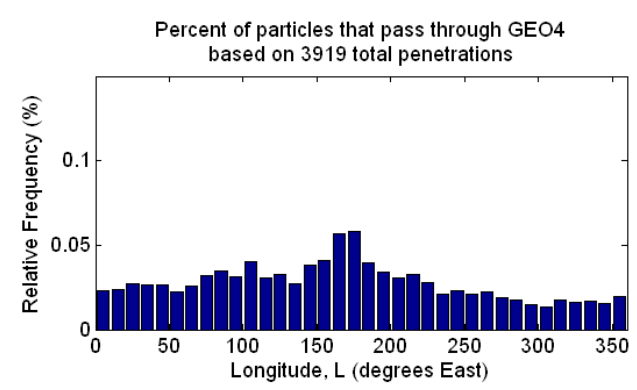

Figure 8.53: Longitude Bin Penetration Percentage

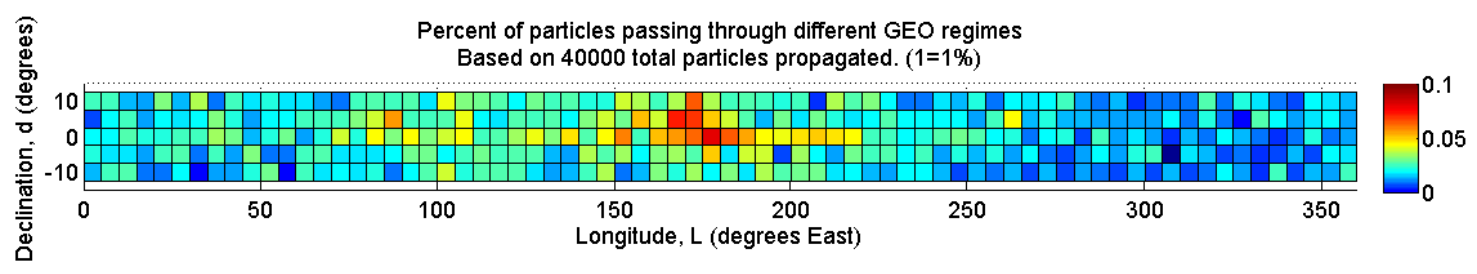

Figure 8.54: Heatmap of Penetrations of Critical Orbit 


\section{Side Effects - GEO15}

The text and graphical output for GEO at 15 degrees is displayed in the following graphs:

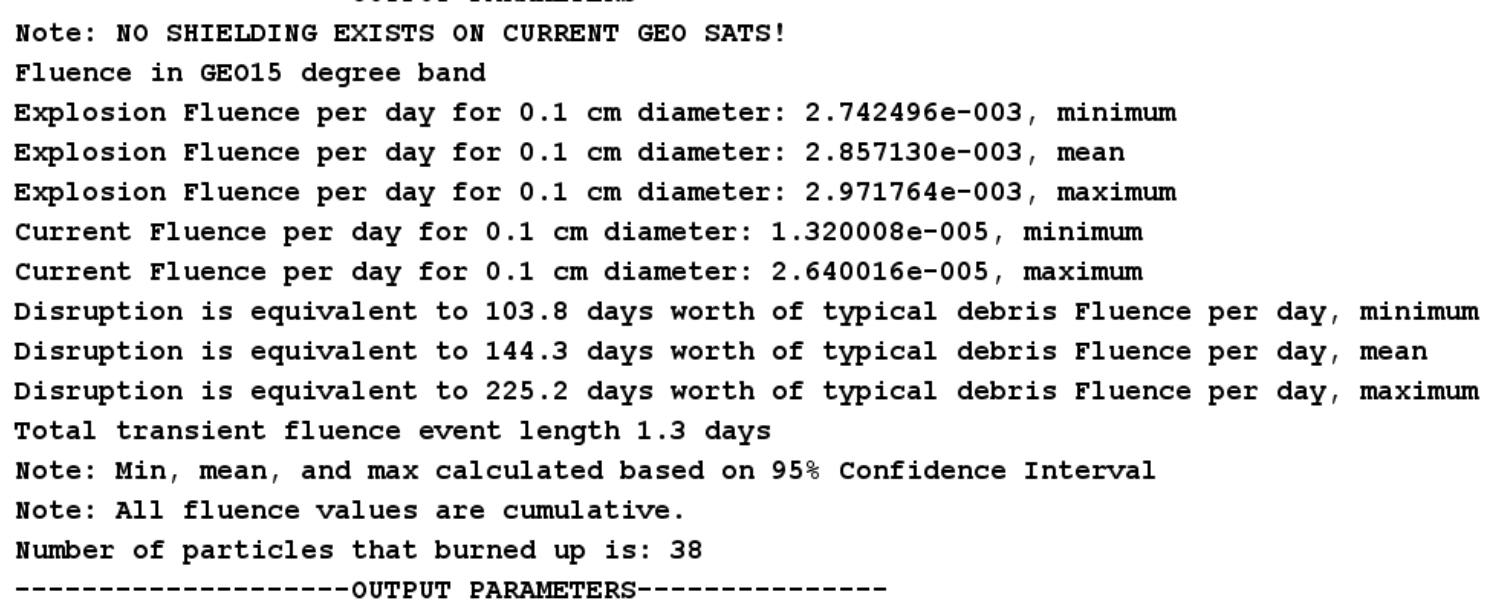

Figure 8.55: MATLAB Command Line Output

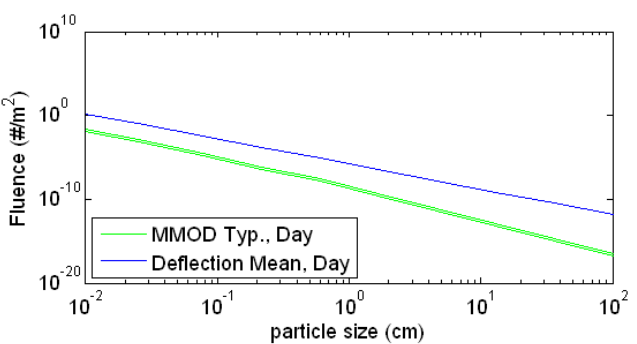

Figure 8.56: Mean MMOD Comparison

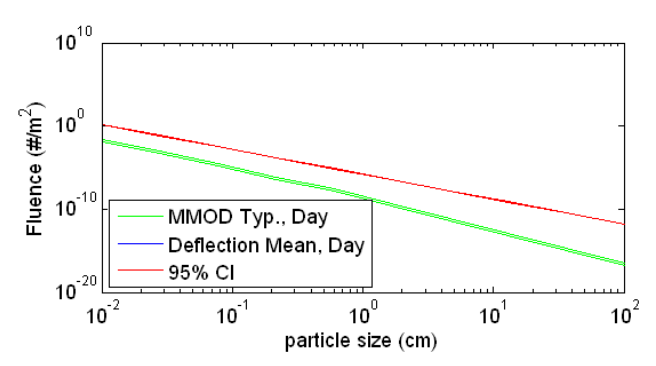

Figure 8.57: MMOD CI Comparison 


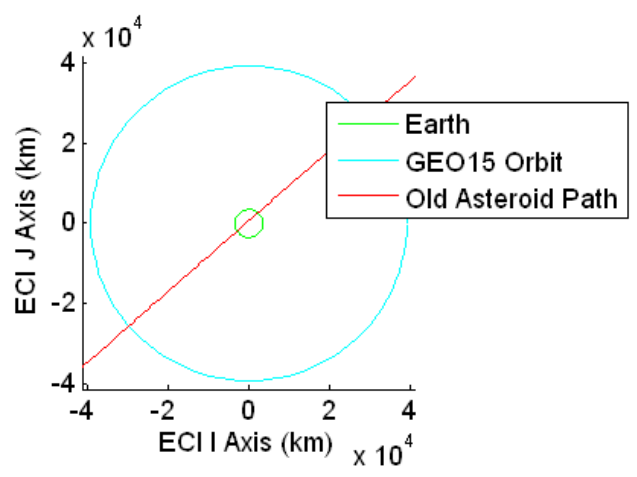

Figure 8.58: Original Asteroid Orbital Path Check

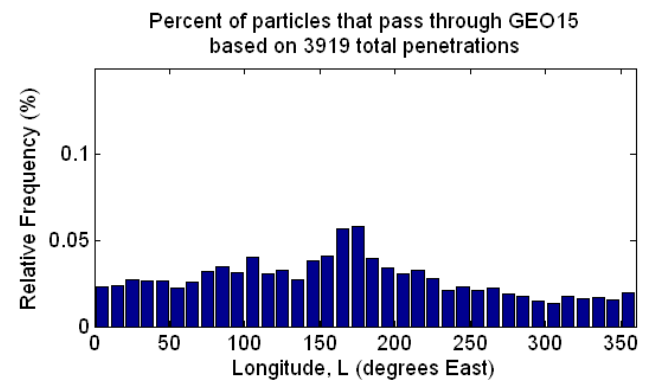

Figure 8.59: Longitude Bin Penetration Percentage

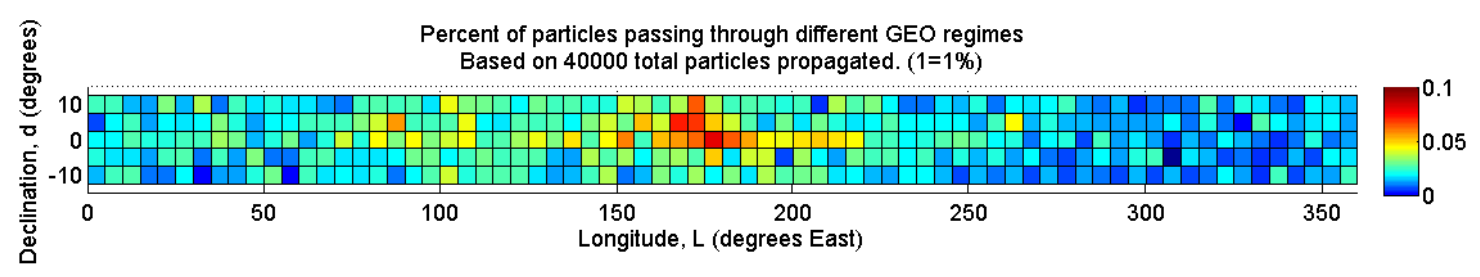

Figure 8.60: Heatmap of Penetrations of Critical Orbit

\subsection{Fic2029-400}

\subsubsection{Results - April 9 Disruption}

Side Effects - LEO425 km

The text and graphical output for LEO at $425 \mathrm{~km}$ altitude is displayed in the following graphs. For detailed graph descriptions on each graph see Section 5.1. 
-------------------OUTPUT PARAMETERS---------------

Note: Critical Diameter assumes LEO sats are shielded

Fluence in LE0425 degree band

Explosion Fluence per day for $1 \mathrm{~cm}$ diameter: 1.829407e-007, minimum

Explosion Fluence per day for $1 \mathrm{~cm}$ diameter: 2.597218e-007, mean

Explosion Fluence per day for $1 \mathrm{~cm}$ diameter: 3.579301e-007, maximum

Current Fluence per day for $1 \mathrm{~cm}$ diameter: 1.509027e-007, minimum

Current Fluence per day for $1 \mathrm{~cm}$ diameter: $1.635626 \mathrm{e}-007$, maximum

Disruption is equivalent to 1.1 days worth of typical debris Fluence per day, minimum

Disruption is equivalent to 1.7 days worth of typical debris Fluence per day, mean

Disruption is equivalent to 2.4 days worth of typical debris Fluence per day, maximum Total transient fluence event length 1.5 days

Note: Min, mean, and max calculated based on $95 \%$ Confidence Interval

Note: All fluence values are cumulative.

Number of particles that burned up is: 47

-------------------OUTPUT PARAMETERS--------------

Figure 8.61: MATLAB Command Line Output

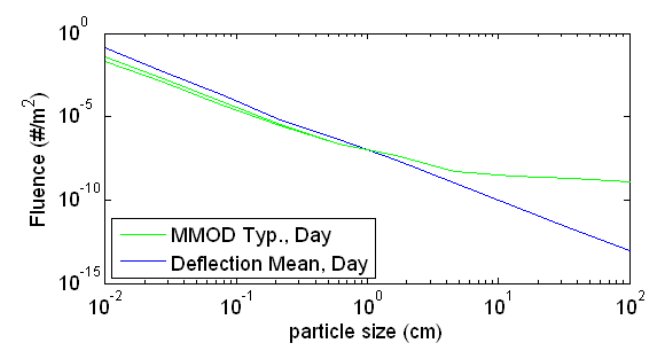

Figure 8.62: Mean MMOD Comparison

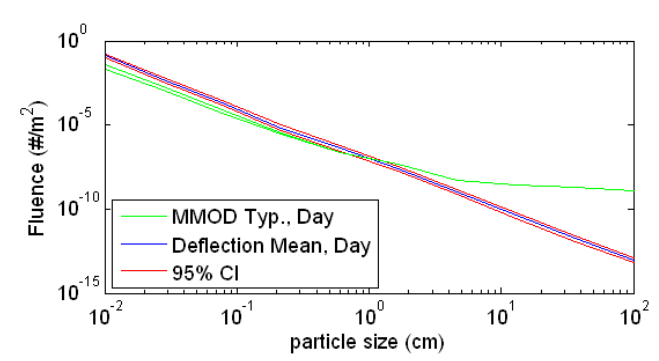

Figure 8.63: MMOD CI Comparison 


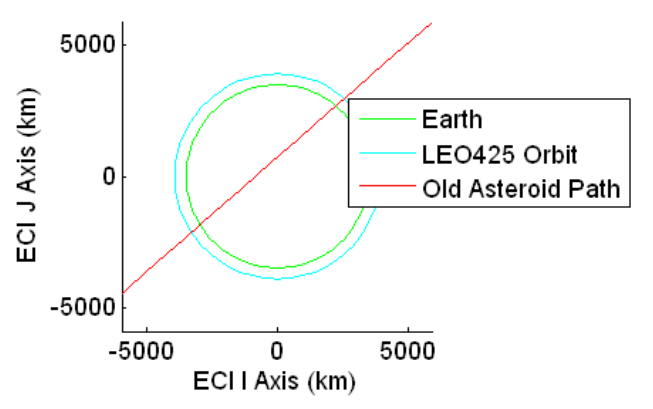

Figure 8.64: Original Asteroid Orbital Path Check

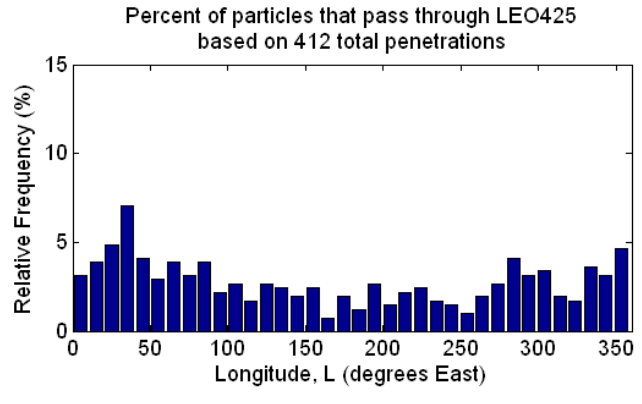

Figure 8.65: Longitude Bin Penetration Percentage

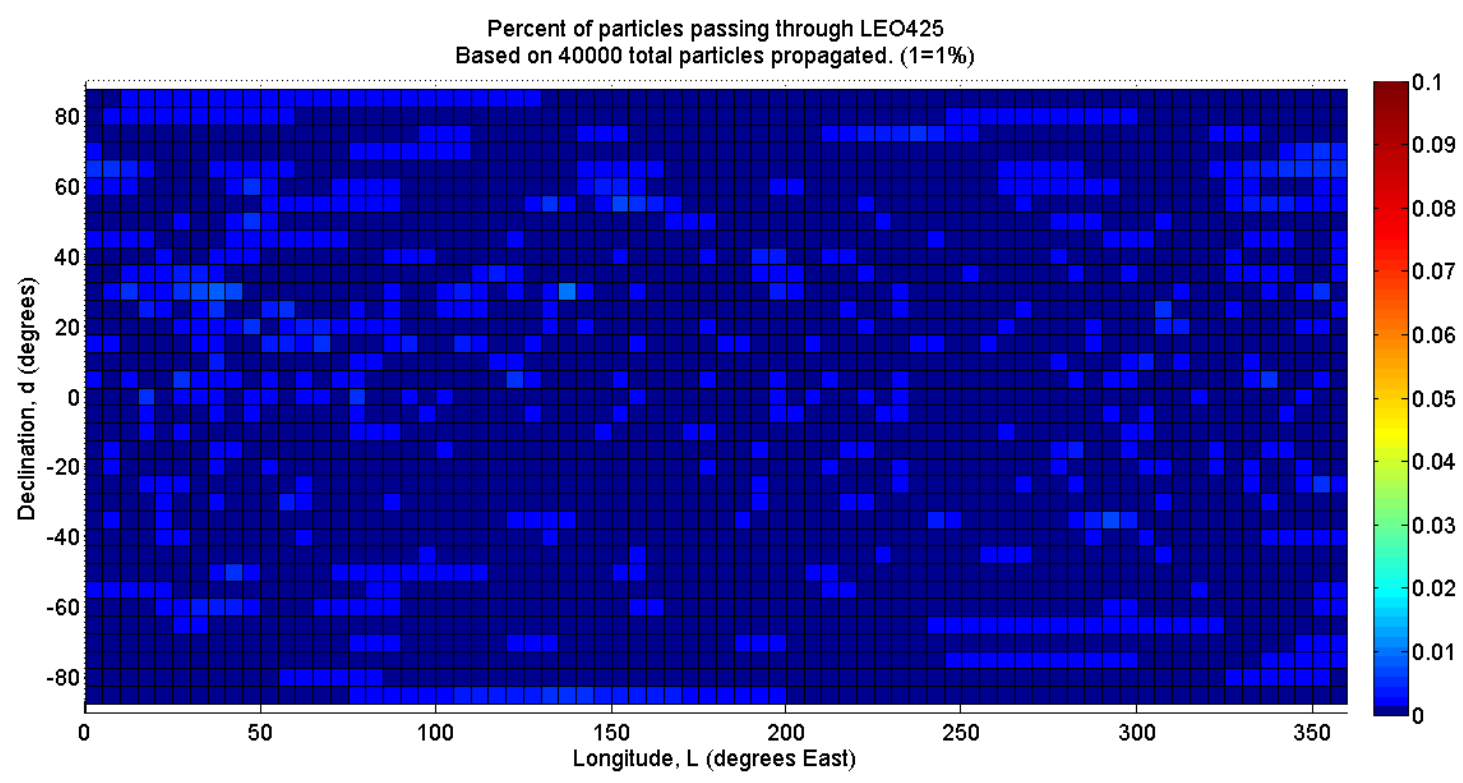

Figure 8.66: Heatmap of Penetrations of Critical Orbit 
Side Effects - GEO1

The text and graphical output for GEO at 1 degree is displayed in the following graphs. For detailed graph descriptions on each graph see Section 5.1.

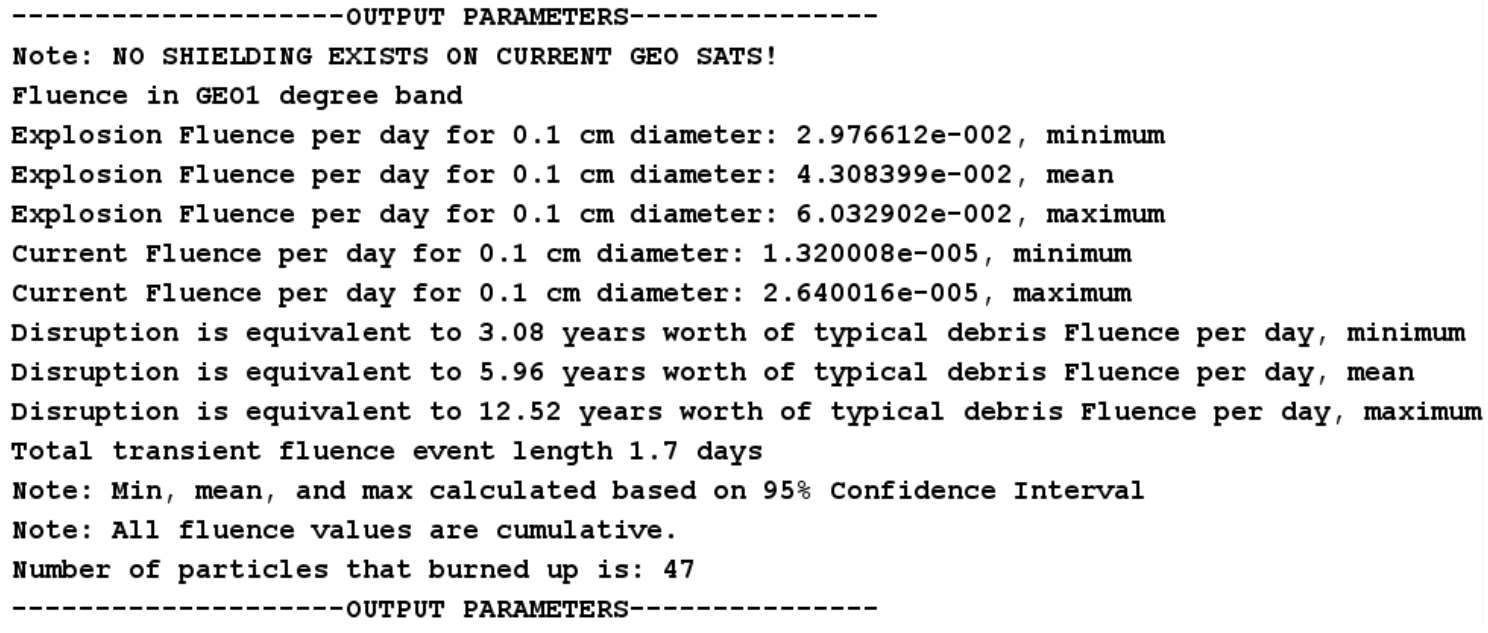

Figure 8.67: MATLAB Command Line Output

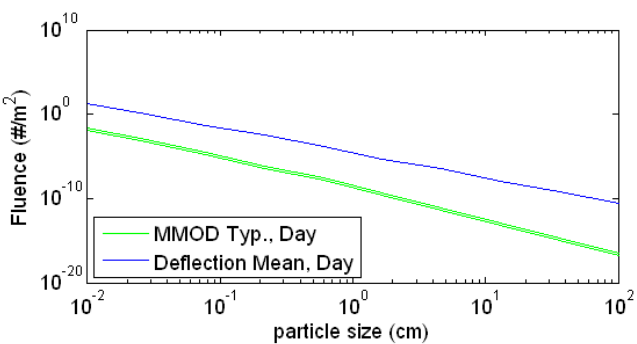

Figure 8.68: Mean MMOD Comparison

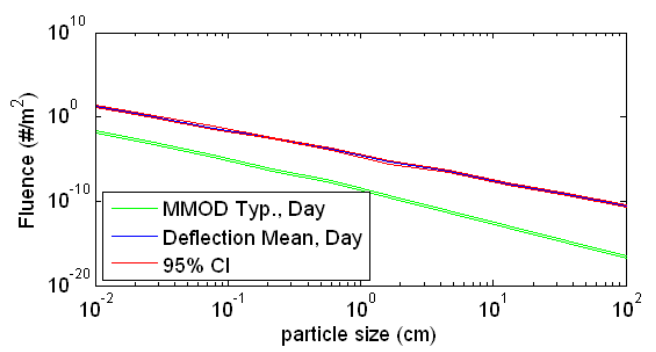

Figure 8.69: MMOD CI Comparison 


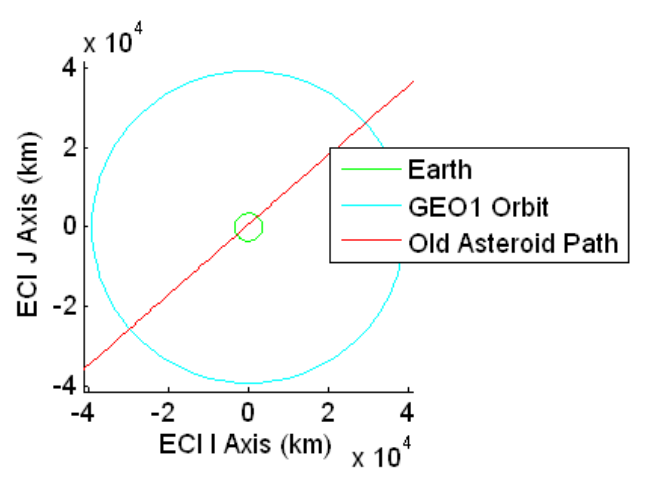

Figure 8.70: Original Asteroid Orbital Path Check

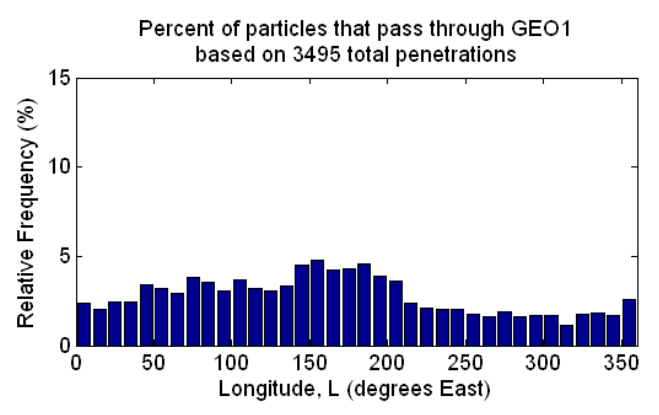

Figure 8.71: Longitude Bin Penetration Percentage

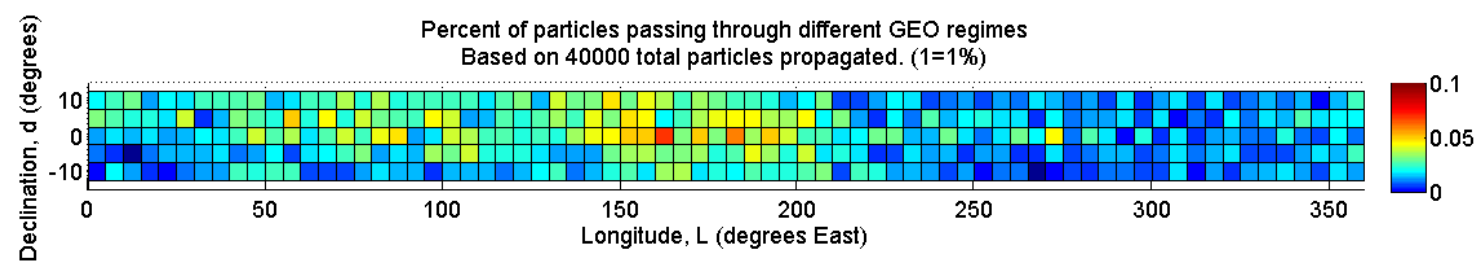

Figure 8.72: Heatmap of Penetrations of Critical Orbit

\subsection{Fic2029-600}

\subsubsection{Results - April 9 Disruption}

Side Effects - LEO425 km

The text and graphical output for LEO at $425 \mathrm{~km}$ is displayed in the following graphs. For detailed graph descriptions on each graph see Section 5.1. 


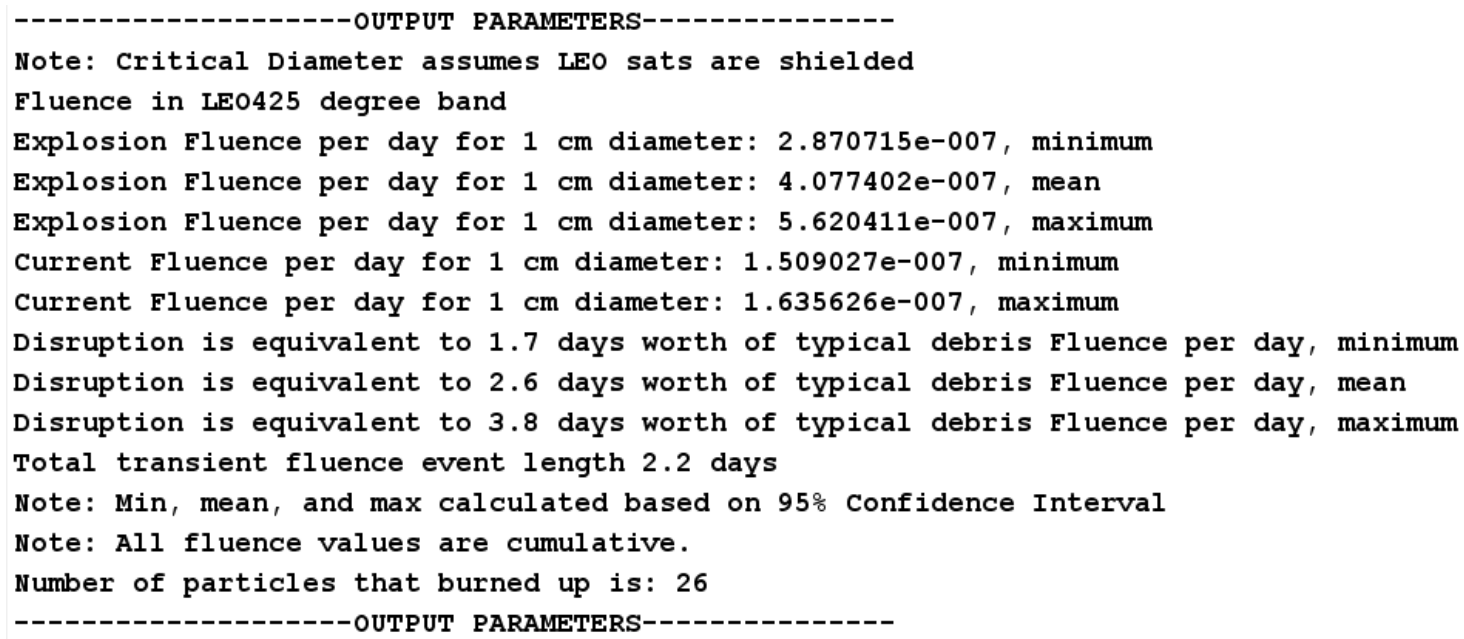

Figure 8.73: MATLAB Command Line Output

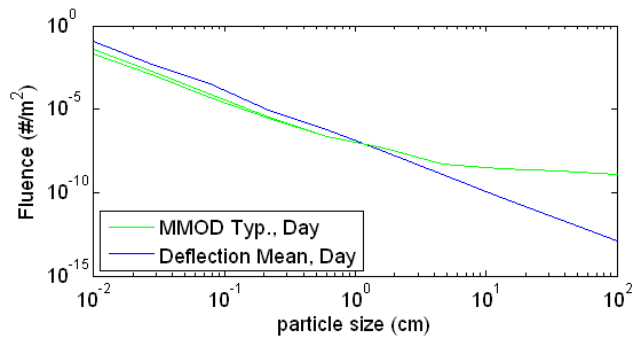

Figure 8.74: Mean MMOD Comparison

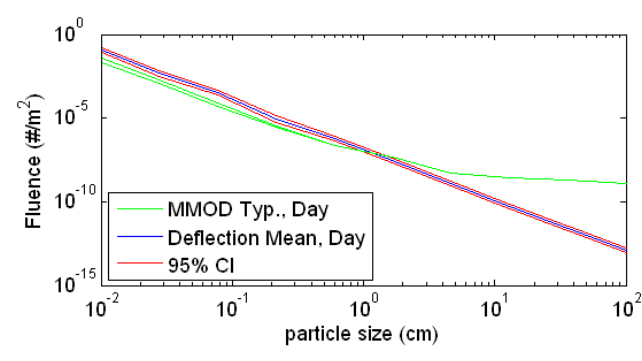

Figure 8.75: MMOD CI Comparison 


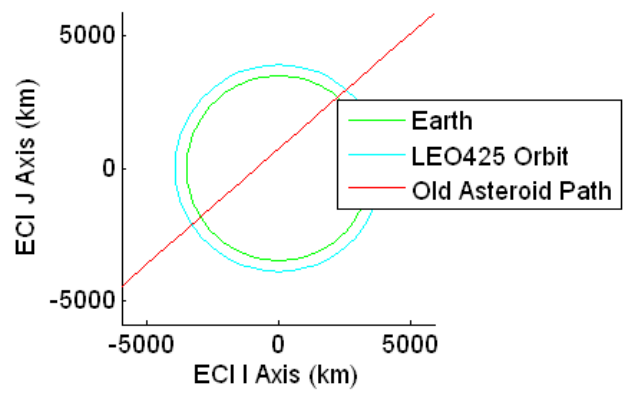

Figure 8.76: Original Asteroid Orbital Path Check

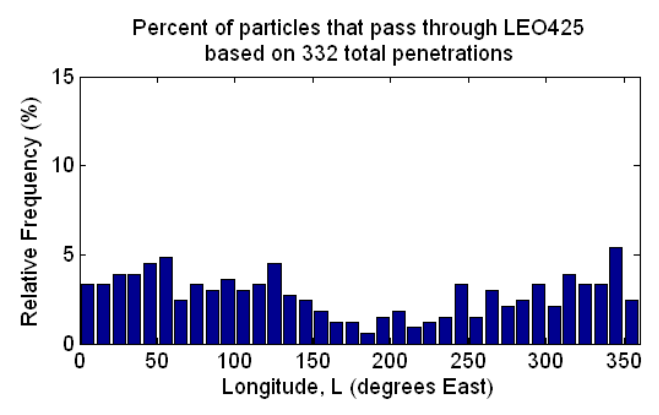

Figure 8.77: Longitude Bin Penetration Percentage

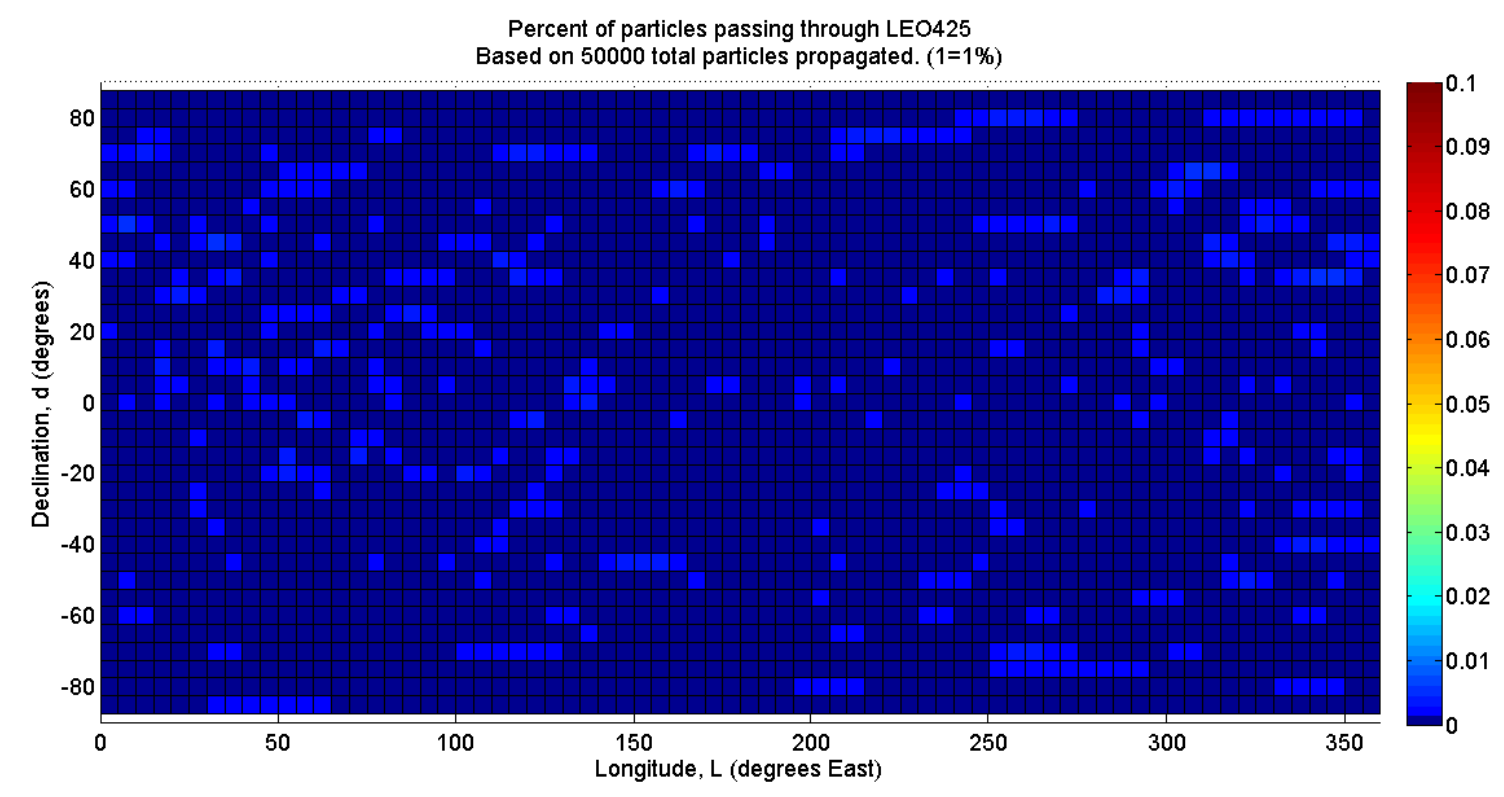

Figure 8.78: Heatmap of Penetrations of Critical Orbit 
Side Effects - GEO1

The text and graphical output for GEO at 1 degree is displayed in the following graphs. For detailed graph descriptions on each graph see Section 5.1.

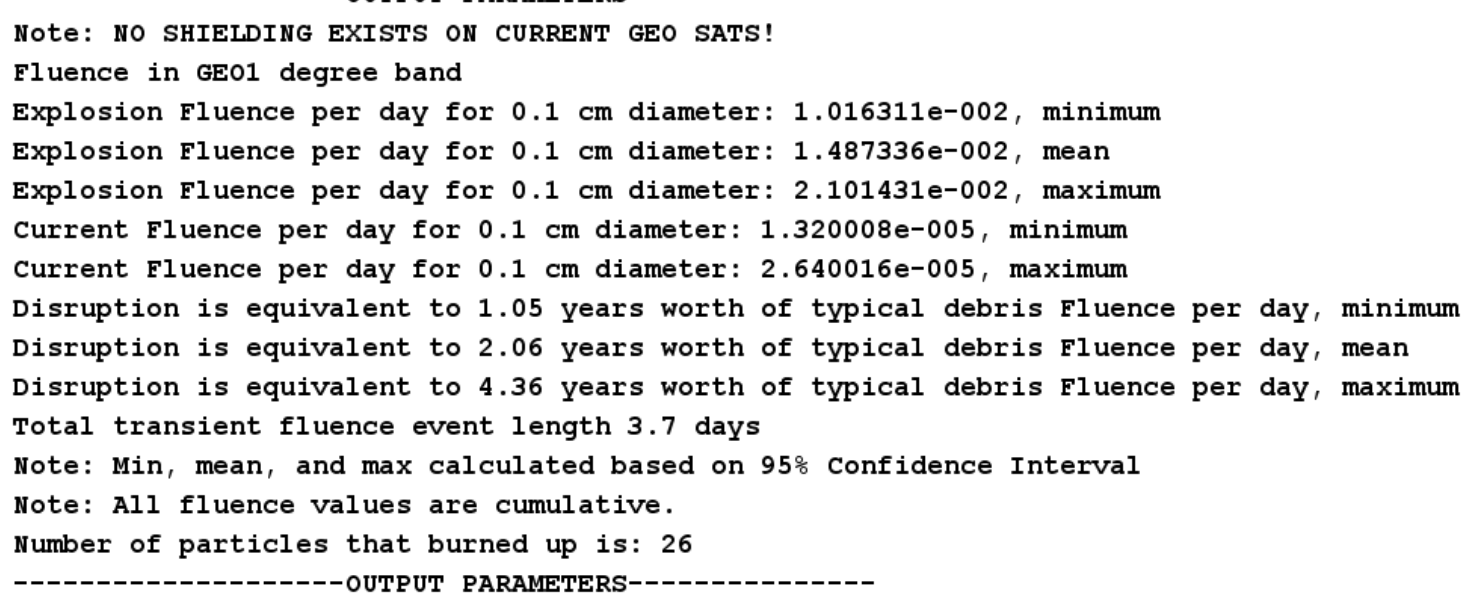

\section{Figure 8.79: MATLAB Command Line Output}

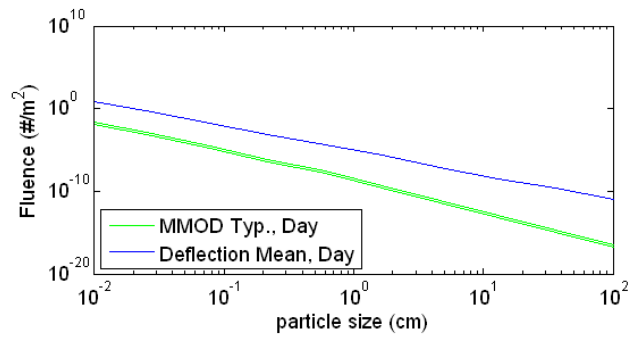

Figure 8.80: Mean MMOD Comparison

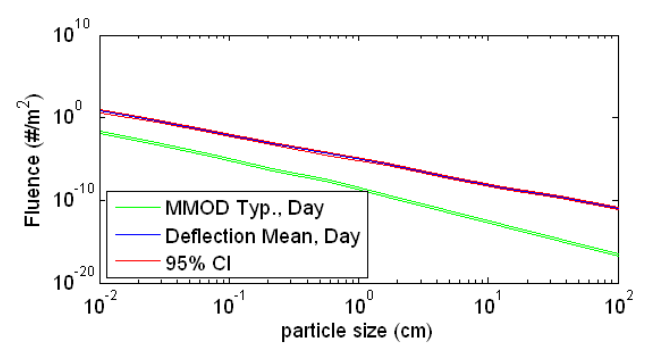

Figure 8.81: MMOD CI Comparison 


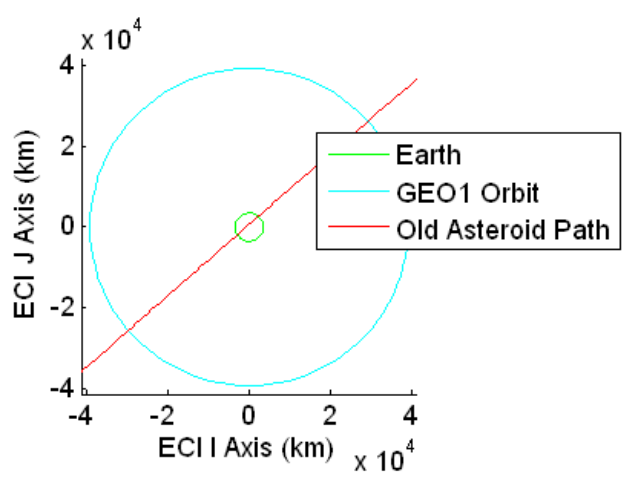

Figure 8.82: Original Asteroid Orbital Path Check

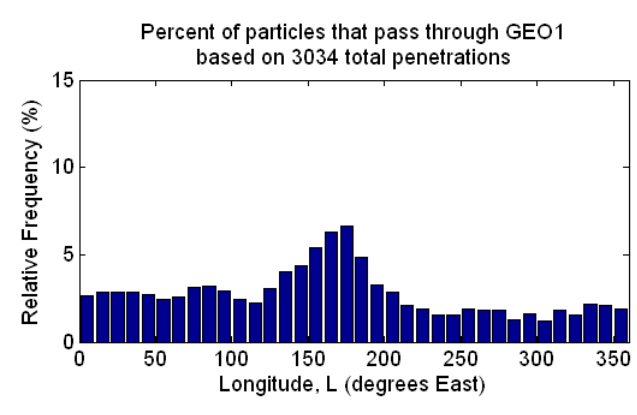

Figure 8.83: Longitude Bin Penetration Percentage

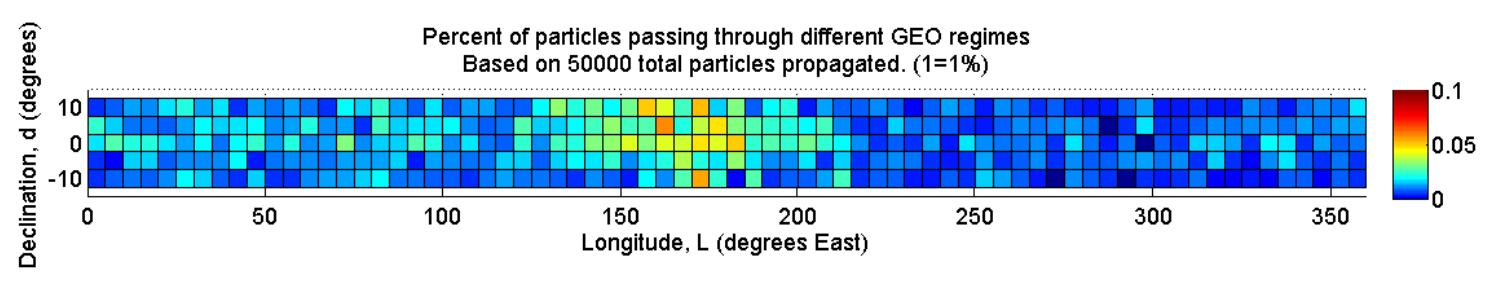

Figure 8.84: Heatmap of Penetrations of Critical Orbit

\subsection{Velocity Distributions}

Highlighted here are the differences between velocity distributions that are provided in the code and their source constraints. Special care should be used when simulating asteroid disruption side effects to ensure that the velocity curve assigned through this program matches the asteroid type and nuclear explosion strength. If substantial differences are evident, such as the case of modeling an asteroid that is 
not Apophis or Itokawa, additional hydrodynamic models should be run to build a new velocity curve file before using this framework to study the side effects.

The Wie1 velocity distribution is based of work published by Dr. Wie and Dearborn [37]. It is a simulation of a spherical Apophis type asteroid $270 \mathrm{~m}$ diameter disrupted by a $300 \mathrm{kt}$ nuclear surface burst explosion.

The Weaver1 velocity distribution simulates the asteroid Itokawa being destroyed by a $500 \mathrm{kt}$ nuclear surface burst explosion on its long end.

The Weaver2 velocity distribution simulates the asteroid Itokawa being destroyed by the $500 \mathrm{kt}$ nuclear surface burst explosion on its short end.

The Weaver3 velocity distribution simulates the asteroid Itokawa being destroyed by a $500 \mathrm{kt}$ nuclear surface burst explosion on its long end. This is similar to the Weaver1 distribution, however, Weaver3 was created with a more up to date version of the RAGE hydrodynamics code.

The Weaver 4 velocity distribution simulates the asteroid Itokawa being destroyed by the 600 kt nuclear surface burst explosion on its long end.

The Weaver5 velocity distribution simulates the asteroid Itokawa being destroyed by the 400 kt nuclear surface burst explosion on its long end. 


\subsection{Supplemental Tables}

Table 8.1: Input Parameters, April 11th Disruption

\begin{tabular}{|c|c|c|}
\hline Explosion Date & {$[2029,4,11,0,0,0]$} & {$[$ year,mm,dd,hh,mm,ss] } \\
Observation Date & {$[2029,4,1,0,0,0]$} & [year,mm,dd,hh,mm,ss] \\
\hline Position Earth (X) & $-9.803428546825277 \mathrm{E}-01$ & AU, Heliocentric \\
Position Earth (Y) & $-1.930901065107917 \mathrm{E}-01$ & AU, Heliocentric \\
Position Earth (Z) & $2.078772183457271 \mathrm{E}-05$ & AU, Heliocentric \\
\hline Velocity Earth (X) & $3.040684386769381 \mathrm{E}-03$ & AU/day, Heliocentric \\
Velocity Earth (Y) & $-1.693819813500764 \mathrm{E}-02$ & AU/day, Heliocentric \\
Velocity Earth (Z) & $8.062826223939135 \mathrm{E}-07$ & AU/day, Heliocentric \\
\hline Position Asteroid (X) & $-1.012505408225337 \mathrm{E}+00$ & AU, Heliocentric \\
Position Asteroid (Y) & $-2.212009168899217 \mathrm{E}-01$ & AU, Heliocentric \\
Position Asteroid (Z) & $-1.209751944049115 \mathrm{E}-02$ & AU, Heliocentric \\
\hline Velocity Asteroid (X) & $5.596108154750269 \mathrm{E}-03$ & AU/day, Heliocentric \\
Velocity Asteroid (Y) & $-1.481348469905512 \mathrm{E}-02$ & AU/day, Heliocentric \\
Velocity Asteroid (Z) & $9.217139513703808 \mathrm{E}-04$ & AU/day, Heliocentric \\
\hline Asteroid Mass & $3.51 \mathrm{E}+10$ & $\mathrm{~kg}$ \\
Asteroid Volume & $3.28736 \mathrm{E}+7$ & $\mathrm{~m}^{3}$ \\
Asteroid Density & 1.05 & $\mathrm{~g} / \mathrm{cm}^{3}$ \\
\hline Largest Remaining Chunk & 20 & meters \\
Explosion Profile & Weaver1.m & string \\
Fragments per Bin & 500 & number \\
\hline Propagation Loops & 8 & - \\
\# Fragment Bins & 10 & - \\
Total \# Fragments & 40,000 & - \\
\hline
\end{tabular}


Table 8.2: Input Parameters, April 9th Disruption

\begin{tabular}{|c|c|c|}
\hline Explosion Date & {$[2029,4,9,0,0,0]$} & {$[$ year,mm,dd,hh,mm,ss] } \\
Observation Date & {$[2029,4,1,0,0,0]$} & year,mm,dd,hh,mm,ss] \\
\hline Position Earth (X) & $-9.803428546825277 \mathrm{E}-01$ & AU, Heliocentric \\
Position Earth (Y) & $-1.930901065107917 \mathrm{E}-01$ & AU, Heliocentric \\
Position Earth (Z) & $2.078772183457271 \mathrm{E}-05$ & AU, Heliocentric \\
\hline Velocity Earth (X) & $3.040684386769381 \mathrm{E}-03$ & AU/day, Heliocentric \\
Velocity Earth (Y) & $-1.693819813500764 \mathrm{E}-02$ & AU/day, Heliocentric \\
Velocity Earth (Z) & $8.062826223939135 \mathrm{E}-07$ & AU/day, Heliocentric \\
\hline Position Asteroid (X) & $-1.012505408225337 \mathrm{E}+00$ & AU, Heliocentric \\
Position Asteroid (Y) & $-2.212009168899217 \mathrm{E}-01$ & AU, Heliocentric \\
Position Asteroid (Z) & $-1.209751944049115 \mathrm{E}-02$ & AU, Heliocentric \\
\hline Velocity Asteroid (X) & $5.596108154750269 \mathrm{E}-03$ & AU/day, Heliocentric \\
Velocity Asteroid (Y) & $-1.481348469905512 \mathrm{E}-02$ & AU/day, Heliocentric \\
Velocity Asteroid (Z) & $9.217139513703808 \mathrm{E}-04$ & AU/day, Heliocentric \\
\hline Asteroid Mass & $3.51 \mathrm{E}+10$ & $\mathrm{~kg}$ \\
Asteroid Volume & $3.28736 \mathrm{E}+7$ & $\mathrm{~m}^{3}$ \\
Asteroid Density & 1.05 & $g / \mathrm{cm}^{3}$ \\
\hline Largest Remaining Chunk & 20 & meters \\
Explosion Profile & Weaver1.m & string \\
Fragments per Bin & 500 & number \\
\hline Propagation Loops & 10 & - \\
\# Fragment Bins & 10 & - \\
Total \# Fragments & 50,000 & \\
\hline
\end{tabular}


Table 8.3: Input Parameters, April 11th Disruption

\begin{tabular}{|c|c|c|}
\hline Explosion Date & {$[2029,4,11,0,0,0]$} & {$[$ year,mm,dd,hh,mm,ss] } \\
Observation Date & {$[2029,4,1,0,0,0]$} & Aear,mm,dd,hh,mm,ss] \\
\hline Position Earth (X) & $-9.803428546825277 \mathrm{E}-01$ & AUliocentric \\
Position Earth (Y) & $-1.930901065107917 \mathrm{E}-01$ & AU, Heliocentric \\
Position Earth (Z) & $2.078772183457271 \mathrm{E}-05$ & AU, Heliocentric \\
\hline Velocity Earth (X) & $3.040684386769381 \mathrm{E}-03$ & AU/day, Heliocentric \\
Velocity Earth (Y) & $-1.693819813500764 \mathrm{E}-02$ & AU/day, Heliocentric \\
Velocity Earth (Z) & $8.062826223939135 \mathrm{E}-07$ & AU/day, Heliocentric \\
\hline Position Asteroid (X) & $-1.012505408225337 \mathrm{E}+00$ & AU, Heliocentric \\
Position Asteroid (Y) & $-2.212009168899217 \mathrm{E}-01$ & AU, Heliocentric \\
Position Asteroid (Z) & $-1.209751944049115 \mathrm{E}-02$ & AU, Heliocentric \\
\hline Velocity Asteroid (X) & $5.596108154750269 \mathrm{E}-03$ & AU/day, Heliocentric \\
Velocity Asteroid (Y) & $-1.481348469905512 \mathrm{E}-02$ & AU/day, Heliocentric \\
Velocity Asteroid (Z) & $9.217139513703808 \mathrm{E}-04$ & AU/day, Heliocentric \\
\hline Asteroid Mass & $3.51 \mathrm{E}+10$ & $\mathrm{~kg}$ \\
Asteroid Volume & $3.28736 \mathrm{E}+7$ & $\mathrm{~m}^{3}$ \\
Asteroid Density & 1.05 & $\mathrm{~g} / \mathrm{cm}^{3}$ \\
\hline Largest Remaining Chunk & 20 & meters \\
Explosion Profile & Weaver1.m & string \\
Fragments per Bin & 500 & number \\
\hline Propagation Loops & 10 & - \\
\# Fragment Bins & 10 & - \\
Total \# Fragments & 50,000 & - \\
\hline
\end{tabular}

\subsection{J2 J6 Coordinate System Transformation}

Vallado's derivation of Zonal Harmonics yields a change in acceleration of the craft due to J2-J6 perturbations. Initially, to move from IJK (Heliocentric) to ECEF (Earth-Centered Earth Fixed) which rotates w/ the earth, the following set of trans- 
formations is used:

$$
R \_i j k=T * R \_e c e f
$$

where $R_{-} i j k$ is the position in the IJK frame and $R_{-} e c e f$ is the position in the ECEF frame. $T$ is the transformation matrix which is given by:

$$
T=\left|\begin{array}{ccc}
\cos (\theta) & -\sin (\theta) & 0 \\
\sin (\theta) & \cos (\theta) & 0 \\
0 & 0 & 1
\end{array}\right|
$$

where $\theta$ is the angle difference between the $\mathrm{X}$ axis of the IJK and ECEF frames. Theta is a function of time. Taking the first time derivative of this function and applying the chain rule yields:

$$
V_{\_} i j k=\dot{T} * R_{e} c e f+T * V_{\_} e c e f
$$

where $V_{-i j k}$ is the velocity of the spacecraft in the IJK frame and $V_{-} e c e f$ is the equivelent velocity in the ECEF frame. $\dot{T}$ is found by taking the time derivatives of the individual parts of $\mathrm{T}$ :

$$
T=\left|\begin{array}{ccc}
-W * \sin (\theta) & -W * \cos (\theta) & 0 \\
W * \cos (\theta) & -W * \sin (\theta) & 0 \\
0 & 0 & 0
\end{array}\right|
$$


where $\mathrm{W}$ is the rotation rate about the $\hat{Z}$ direction and is considered to be constant. Taking the time derivative and applying the chain rule again yields:

$$
A \_i j k=T d d * R \_e c e f+T d o t * V \_e c e f+T d o t * V \_e c e f+T * A \_e c e f
$$

where $A_{-} i j k$ is the acceleration of the spacecraft in the IJK and $A_{-} e c e f$ is the equivelent acceleration in the ECEF. $\ddot{T}$ is the second time derivative of the T matrix.

$$
T=\left|\begin{array}{ccc}
-\dot{W} * \sin (\theta)-W^{2} * \cos (\theta) & -\dot{W} * \cos (\theta)+W^{2} * \sin (\theta) & 0 \\
\dot{W} * \cos (\theta)-W^{2} * \sin (\theta) & -\dot{W} * \sin (\theta)-W^{2} * \cos (\theta) & 0 \\
0 & 0 & 0
\end{array}\right|
$$

Since there is not acceleration associated with $\mathrm{W}, \dot{W}$ is zero. This simplifies $\ddot{T}$ to:

$$
T=\left|\begin{array}{ccc}
-W^{2} * \cos (\theta) & +W^{2} * \sin (\theta) & 0 \\
-W^{2} * \sin (\theta) & -W^{2} * \cos (\theta) & 0 \\
0 & 0 & 0
\end{array}\right|
$$

Now an expression for the change in acceleration in IJK due to J2-J6 perturbations must be found.

$$
D e l \_A \_i j k=A \_i j k_{-} 1-A_{-} i j k_{-} 0
$$




$$
A \_i j k_{-} 0=T d d * R \_e c e f+2 * T d o t * V \_e c e f+T * A \_e c e f \_0
$$

$$
A \_i j k_{\_} 1=T d d * R \_e c e f+2 * T d o t * V \_e c e f+T *\left(A \_e c e f \_0+D e l \_A \_e c e f\right)
$$

Plugging these two equations into the first one, it is noted that regardless of the initial acceleration in the IJK, the equation for Del_A_ijk simplifies to:

$$
D e l \_A \_i j k=T * D e l \_A \_e c e f
$$

Where Del_A_ecef is solved for using the ZonalHarmonic.m function. 


\begin{tabular}{|c|c|}
\hline $\begin{array}{l}\text { Absolute Magnitude } \\
\qquad(\mathrm{H})\end{array}$ & $\begin{array}{c}\text { Diameter } \\
\text { (km = kilometers }) \\
\text { ( } \mathrm{m}=\text { meters })\end{array}$ \\
\hline 3.0 & $670 \mathrm{~km}-1490 \mathrm{~km}$ \\
\hline 3.5 & $530 \mathrm{~km}-1190 \mathrm{~km}$ \\
\hline 4.0 & $420 \mathrm{~km}-940 \mathrm{~km}$ \\
\hline 4.5 & $330 \mathrm{~km}-750 \mathrm{~km}$ \\
\hline 5.0 & $270 \mathrm{~km}-590 \mathrm{~km}$ \\
\hline 5.5 & $210 \mathrm{~km}-470 \mathrm{~km}$ \\
\hline 6.0 & $170 \mathrm{~km}-380 \mathrm{~km}$ \\
\hline 6.5 & $130 \mathrm{~km}-300 \mathrm{~km}$ \\
\hline 7.0 & $110 \mathrm{~km}-240 \mathrm{~km}$ \\
\hline 7.5 & $85 \mathrm{~km}-190 \mathrm{~km}$ \\
\hline 8.0 & $65 \mathrm{~km}-150 \mathrm{~km}$ \\
\hline 8.5 & $50 \mathrm{~km}-120 \mathrm{~km}$ \\
\hline 9.0 & $40 \mathrm{~km}-90 \mathrm{~km}$ \\
\hline 9.5 & $35 \mathrm{~km}-75 \mathrm{~km}$ \\
\hline 10.0 & $25 \mathrm{~km}-60 \mathrm{~km}$ \\
\hline 10.5 & $20 \mathrm{~km}-50 \mathrm{~km}$ \\
\hline 11.0 & $15 \mathrm{~km}-40 \mathrm{~km}$ \\
\hline 11.5 & $13 \mathrm{~km}-30 \mathrm{~km}$ \\
\hline 12.0 & $11 \mathrm{~km}-24 \mathrm{~km}$ \\
\hline 12.5 & $8 \mathrm{~km}-19 \mathrm{~km}$ \\
\hline 13.0 & $7 \mathrm{~km}-15 \mathrm{~km}$ \\
\hline 13.5 & $5 \mathrm{~km}-12 \mathrm{~km}$ \\
\hline 14.0 & $4 \mathrm{~km}-9 \mathrm{~km}$ \\
\hline 14.5 & $3 \mathrm{~km}-7 \mathrm{~km}$ \\
\hline 15.0 & $3 \mathrm{~km}-6 \mathrm{~km}$ \\
\hline 15.0 & $3 \mathrm{~km}-6 \mathrm{~km}$ \\
\hline 15.5 & $2 \mathrm{~km}-5 \mathrm{~km}$ \\
\hline 16.0 & $2 \mathrm{~km}-4 \mathrm{~km}$ \\
\hline
\end{tabular}

Table 8.4: Absolute Magnitude to Diameter Conversion[30] 


\begin{tabular}{|c|c|}
\hline $\begin{array}{l}\text { Absolute Magnitude } \\
\qquad(\mathrm{H})\end{array}$ & $\begin{array}{c}\text { Diameter } \\
\text { (km = kilometers }) \\
(\mathrm{m}=\text { meters })\end{array}$ \\
\hline 16.5 & $1 \mathrm{~km}-3 \mathrm{~km}$ \\
\hline 17.0 & $1 \mathrm{~km}-2 \mathrm{~km}$ \\
\hline 17.5 & $1 \mathrm{~km}-2 \mathrm{~km}$ \\
\hline 18.0 & $670 m-1500 m$ \\
\hline 18.5 & $530 m-1200 m$ \\
\hline 19.0 & $420 m-940 m$ \\
\hline 19.5 & $330 m-750 m$ \\
\hline 20.0 & $270 m-590 m$ \\
\hline 20.5 & $210 m-470 m$ \\
\hline 21.0 & $170 m-380 m$ \\
\hline 21.5 & $130 m-300 m$ \\
\hline 22.0 & $110 m-240 m$ \\
\hline 22.5 & $85 m-190 m$ \\
\hline 23.0 & $65 m-150 m$ \\
\hline 23.5 & $50 m-120 m$ \\
\hline 24.0 & $40 m-95 m$ \\
\hline 24.5 & $35 m-75 m$ \\
\hline 25.0 & $25 m-60 m$ \\
\hline 25.5 & $20 m-50 m$ \\
\hline 26.0 & $17 m-37 m$ \\
\hline 26.5 & $13 m-30 m$ \\
\hline 27.0 & $11 m-24 m$ \\
\hline 27.5 & $8 m-19 m$ \\
\hline 28.0 & $7 m-15 m$ \\
\hline 28.5 & $5 m-12 m$ \\
\hline 29.0 & $4 m-9 m$ \\
\hline 29.5 & $3 m-7 m$ \\
\hline 30.0 & $3 m-6 m$ \\
\hline
\end{tabular}

Table 8.5: Absolute Magnitude to Diameter Conversion[30] 


\section{Bibliography}

[1] China confirms satellite downed. $\quad B B C$ News, January 2007. http://news.bbc.co.uk/2/hi/asiapacific/6289519.stm Accessed May 15, 2013.

[2] Natural Impact Hazard (Asteroid Strike) Interagency Deliberate Planning Exercise After Action Report. US Air Force, Directorate of Strategic Planning, AF/A8XC, December 2008.

[3] Us missile hits 'toxic satellite'. $\quad B B C$ News, February 2008. http://news.bbc.co.uk/2/hi/7254540.stm Accessed May 15, 2013.

[4] T. Ahrens and A. Harris. Deflection and fragmentation of near-earth asteroids. Nature, December 1992.

[5] J. Amos. Final skynet satellite launched. BBC News, June 2008. http://news.bbc.co.uk/2/hi/science/nature/7451867.stm Accessed May 15, 2013.

[6] J. Appelbaum and D. Flood. Solar radiation on mars. NASA Technical Memorandum, 1989. 
[7] R. R. Bate, D. D. Mueller, and J. E. White. Fundamentals of Astrodynamics. Dover Publications, 1971.

[8] M. Bauer. Herschel intercepts asteroid apophis. January 2013. http://www.esa.int/Our_Activities/Space_Science/Herschel Accessed May 15, 2013.

[9] P. Black, B. Milanova, and L. Smith-Spark. Russian meteor blast injures at least 1,000 people, authorities say. CNN, February 2013. http://edition.cnn.com/2013/02/15/world/europe/russiameteorshower Accessed May 15, 2013.

[10] J. W. Canan. Conversations with Martin C. Faga. Aerospace America, May 2012.

[11] V. A. Chobotov. Orbital Mechanics. AIAA Education Series, third edition, 2002.

[12] E. L. Christiansen and D. M. Lear. Micrometeoroid and orbital debris environment and hypervelocity shields. Technical report, NASA Johnson Space Center, 2012. http://ntrs.nasa.gov/archive/nasa/casi.ntrs.nasa.gov/20120002584_2012002224.pdf Accessed May 15, 2013.

[13] S. Close. Space invaders: Shooting stars can shoot down satellites. IEEE Spectrum, April 2010. 
[14] H. D. Curtis. Orbital Mechanics for Engineering Students. ButtersworthHeineman, second edition, 2009.

[15] D. Dearborn, S. Patenaude, and R. Managan. The use of nuclear explosives to disrupt or divert asteroids. Planetary Defence Conference, 2007.

[16] I. Giblin, G. Martelli, P. Farinella, P. Paolicchi, M. D. Martino, and P. N. Smith. The properties of fragments from catastrophic disruption events. ICARUS, 134:77-112, 1998.

[17] S. Gong, J. Li, and H. BaoYin. Formation flying solar-sail gravity tractors in displaced orbit for towing near-earth asteroids. Celest Mech Dyn Astr, 105:159177, 2009.

[18] T. Jones. Finding neos: Stepping stones for human exploration. Aerospace America, 9, September 2011.

[19] J. A. Kaplan. Russian meteorite 1000 times bigger than originally thought. FOX News, February 2013. http://www.foxnews.com/science/2013/02/19/russianmeteorite1000timesbiggerthanoriginallythought Accessed May 15, 2013.

[20] B. Kaplinger, B. Wie, and D. Dearborn. Preliminary results for high-fidelity modeling and simulation of orbital dispersion of asteroids disrupted by nuclear explosives. AIAA/AAS Astrodynamics Specialist Conference, 2010.

[21] B. Kaplinger, B. Wie, and D. Dearborn. Nuclear fragmentation/dispersion modeling and simulation of hazardous near-earth objects. IAA Planetary Defense Conference, 2011. 
[22] D. Kessler and B. Cour-Palais. Collision frequency of artificial satellites: The creation of a debris belt. Journal of Geophysical Research, 83(A6), June 1978.

[23] J.-C. Liou, M. J. Matney, P. D. Anz-Meador, D. Kessler, M. Jansen, and J. R. Theall. The new nasa orbital debris engineering model ordem2000. Technical report, NASA, 2002. NASA/TP-2002-210780.

[24] NASA. Near-earth object survey and deflection analysis of alternatives. NASA Report to Congress, 2007.

[25] G. E. Peterson and D. K. Lynch. Micrometeoroid and orbital debris environments for the international space station. Technical Report TR-2008(8570)-1, US Air Force, December 2007.

[26] V. L. Pisacane. The space environment and its effects on space systems. AIAA Education Series, 2008.

[27] J. Sanchez, M. Vasile, and G. Radice. Consequences of asteroid fragmentation during impact hazard mitigation. Guidance, Control, and Dynamics, 33(1), Jan-Feb 2010.

[28] R. J. Suggs. Future solar activity estimates for used in prediction of space environmental effects on spacecraft orbital lifetime and performance. NASA Marshall Spaceflight Center, May 2013.

$\begin{array}{cccccc}\text { [29] Associated } & \text { Press. } & 400 & \text { hurt } & \text { when } & \text { mete- } \\ \text { orite falls } & \text { in } & \text { russia. } & \text { silive.com, } & \text { February } & 2013 .\end{array}$ 
http://www.silive.com/news/index.ssf/2013/02/400_hurt_when_meteorite_falls.html Accessed May 15, 2013.

[30] Jet Propulsion Laboratory Staff. Absolute magnitude (h). January 2013. http://neo.jpl.nasa.gov/glossary/h.html Accessed May 15, 2013.

[31] Jet Propulsion Laboratory Staff. Horizons web-interface. May 2013. http://ssd.jpl.nasa.gov/horizons.cgi Accessed May 15, 2013.

[32] NASA Orbital Debris Program Office. Orbital debris graphics. January 2013. http://orbitaldebris.jsc.nasa.gov/photogallery/beehives.html\#leo Accessed May 15, 2013.

[33] A. C. Tribble. The Space Environment: Implications for Spacecraft Design. Princeton University Press, 2003.

[34] D. A. Vallado. Fundamentals of astrodynamics and applications third edition. 2007.

[35] R. Weaver. Breakthroughs - los alamos national lab asteroid killer simulation. Technical report, Los Alamos National Labs, 2012. http://youtu.be/T1yoiE6U5c Accessed May 15, 201.

[36] B. Wie. Astrodynamic fundamentals for deflecting hazardous near-earth objects. IAC, 2009.

[37] B. Wie and D. Dearborn. Earth-impact modelling and analysis of a near-earth 
object fragmented and ddispersed by nuclear subsurface explosions. AAS, 137, 2010. 